\title{
2017 Thank you to our reviewers
}

The Editor would like to publicly acknowledge the people listed below who served as reviewers on the journal during 2017. Without their efforts the quality of the journal could not be sustained.

Aacharya Ramesh

Aadland Eivind

Aakre Ca

Aapro M

Aaro Leif

Aarsland Dag

Aasbjerg K

Aasdahl Lene

Aasekjer Katrine

Abas Melanie

Abbas Ahmed

Abbey Hilary

Abbott Jason

Abbott Tom

Abbruzzese Giovanni

Abdel-Aleem Hany

Abdel-Karim Abdul-

Rahman

Abdelazim Ibrahim

Abdool Karim Salim

Abdool Karim Salim S

Abdul Aziz Zariah

Abdullah Fizan

Abdullah Abu

Abdullah Adina

Abdullah Asnawi

Abdullah Hairil

Abe Takayuki

Abel Gary

Åberg N David

Abernathy James $\mathrm{H}$

Abey Sally

Abheiden Cnh

Abhishek A

Abiiro Gilbert Abotisem

Abitbol Carolyn

Ablin Jacob N

Abolhassani Nazanin

Abou-El-Enein Mohamed

Aboumarzouk Omar M

Abraha Iosief

Abraham Joanna

Abrahams-Gessel Shafika

Abramson John

Abroug Fekri

Abu-Raddad Laith

Abu-Yaghi Nakhleh E

Abu-Zidan Fikri

Abughosh Susan
Abumuaileq Rami

Riziq-Yousef

Abuya Timothy

Acar M

Accordino Federica

Acharya Moulinath

Acheson Austin

Achoki Tom

Acker Jason

Acosta Stefan

Adachi T

Adam Ishag

Adam Mary

Adam Safwaan

Adami Gian

Adamik Barbara

Adams Brian

Adams J

Adams Lisa V

Addis Antonio

Addo Juliet

Adebajo Ade

Adegbija Odewumi

Adegboye Oyelola

Adelman Eric

Adelman Ronald

Adeloye Davies

Adeniyi Oladele Vincent

Adewuya Abiodun

Adhikari Neill

Adisa Rasaq

Adkoli Bv

Adler Alma

Adolfsson L

Adriaanse Marcel

Adriaenssens Jef

Afifi Abdelmonem

Agarwal Mukesh

Agarwal Seema

Ageberg Eva

Agel Julie

Agius Paul

Agner Tove

Agnew Cakil

Agogo George

Agoritsas Thomas

Agosto Caterina

Agrawal Sahil

Agrawal Sutapa

Aguiar Magda
Aguilar Ferrandiz

Encarnacion

Aguilar C

Agulnik Asya

Agustí Benito Cristina

Agyemang Charles

Agyepong Irene

Ahaus Kees

Ahlehoff Ole

Ahlquist David

Ahmed Ali

Ahmed Fayyaz

Ahmed Khalid

Ahmed Samrah

Ahn Hanjong

Ahrens Katherine

Ahuja Kiran

Aichele Stephen

Aida Jun

Aidala Angela

Aiga Hirotsugu

Aiken Abigail

Aiken Catherine

Aitken Leanne

Aitken Stuart

Ajani Andrew

Ajili Faouzia

Akano Obinna

Akay Serhat

Akchurin Oleh

Akdemir Nesibe

Akem Dimala Christian

Akins Ralitsa

Akinyemi J

Akman Mehmet

Akmatov Manas

Akparibo Robert

Akram Umair

Aksoy Mehmet

Al Sayah Fatima

Al-Gobari Muaamar

Al-Hasan Majdi N

Al-Hendy Ayman

Al-jefout Moamar

Al-Khaled M

Al-Naggar Redhwan

Ahmed

Al-Okka Randa

Al-Sughayer Mona

Al-Tannir Mohamad
Al-Wazaify Mayyada

Aladeniyi Isaac

Alali Aziz

Alamgir Hasanat

Alamian Arsham

Alamri Y

Alanio A

Alarcon Walter

Alba Carolina

Albarqouni Loai

Alberici Federico

Alberti Hugh

Albor Christo

Albritton Karen

Alderson Sarah

Ale Olagoke

Aleksandrova Krasimira

Alemi Qais

Alene Kefyalew Addis

Aletras Vassilios

Alfieri Alex

Ali Mohammed Sanni

Ali Huma

Ali Iftikhar

Ali Raghib

Alimoglu Orhan

Alisic Eva

Alizadeh Mohammad

Aljameel Albandary

Alkhatib Ahmad

Allamani Allaman

Allebeck Peter

Allem Jon-Patrick

Allen Andrew

Allen C

Allen Isabel

Allen Kerry

Allgar Victoria

Allman-Farinelli Margaret

Allwright Shane

Almadi T

Almasi-Hashiani Amir

Almasri Jehad

Almdal Thomas

Almeda Ortega Jesús

Almeida João

Almendra Ricardo

Almirall José

Alonso Wladimir

Alouini S 
Alpizar-Rodriguez D

Alpsoy Erkan

Alshaikh M

Alshehri Mansour

Alterman Ron L

Althabe F

Altman B

Altman Donald S

Altobelli Emma

Altuğ Filiz

Alvarez-Alvarez I

Álvarez-Bueno Celia

Alvarez-Moya Eva

Alves Elisabete

Alves João

Aly Markus

Alzahrani Musa

Alzahrani Sami

Amar David

Ambresin Gilles

Ambroggio Lilliam

Ambrosio Giuseppe

Ambrosy Andrew

Amianto Federico

Amin Nordin Syafinaz

Amin Hardik

Aminde Leopold

Amir Lisa

Amir Yufitriana

Amiri Motasahm

Amital Howard

Amland R

Ammirati E

Amoah Padmore

Amoaku W

Ampat George

Amugsi Dickson

Anaf Julia

Ananiev J

Anaya Juan-Manuel

Anchala R

Andaluz Norberto

Andersen Zorana

Jovanovic

Andersen Anne-Marie

Anderson Annie

Anderson Craig

Anderson Emma Louise

Anderson Fm

Anderson Kristen

Anderson Peter

Anderson Vivki

Andersson Thomas

Andò Giuseppe

Andrade Ortega Juan

Alfonso

Andrasik Mp

Andre Florian
Andreassen Bettina Kulle

Andreassi MG

Andreassi Maria

Andreeva Tatiana

Andrew Michael

Andrews Nicole

Andrews S

Andriessen K

Andriessen Karl

Ang Pei

Angele Mk

Angelillo Italo F

Angell Blake

Angelucci E

Angerud Kh

Angioi Manuela

Anil S

Animaw Worku

Anota Amélie

Anothaisintawee

Thunyarat

Anpalahan Mahesan

Ansa Benjamin

Ansah John

Ansari Nasratullah

Anselmi Laura

Ansmann Lena

Anstice Nicola

Anthony Jim

Anttonen Vuokko

Antunes J

Anty Rodolphe

Anutrakulchai Sirirat

Aoun Bahous S

Apollonio Dorothy

Appelbaum Paul S

Appelqvist-Schmidlechner Kaija

Appiah Duke

Apps Lindsay

Apps Michael

Arai L

Arandjelović Ognjen

Arato Teruyo

Araújo J

Araújo Carla

Arbel Ronen

Arber Anne

Arbo Antonio

Arbon Paul

Arbustini Eloisa

Archer Julian

Archibald Daryll

Arcos Estela

Arday David

Ardern Chris

Ardern Cl

Arditi Chantal
Aregbesola Alex

Arem Hannah

Aremu Olatunde

Ariake Kyohei

Arim Rubab

Arima Kazuhiko

Arimoto Takanori

Aris Izzuddin Bin Mohd

Ariyoshi Koya

Arkema Elizabeth

Arliani Gustavo

Armstrong Ben

Armstrong Gail

Armstrong Mary Anne

Armstrong $\mathrm{Mj}$

Armstrong Rebecca

Arnal Jean-Michel

Arnaout Maha

Arnedo-Pena Alberto

Arnet Isabelle

Arnetz Bengt

Arnetz Judy

Arnold-Reed Diane

Arnold W David

Aro Arja

Aromataris Edoardo

Arora Rakesh C

Arora Rc

Arslan Mustafa

Arthur Eric

Artus Majid

Arulkumaran Nish

Arundell Lauren

Arvay Melissa

Aryal Kk

Asai T

Asaithambi Ganesh

Asakawa Tetsuya

Ascione Raimondo

Ashby Sue

Asher Elad

Asher M

Ashleigh Raymond

Ashorn Ulla

Ashurst John

Asiki Gershim

Askim Torunn

Aslani Parisa

Aspinall Peter

Assari Shervin

Assi Valentina

Assis David

Astbury Nerys

Asthworth Mark

Aswathikutty Aswathikutty

Asztalos E

Atchison Kathryn

Atherton Helen
Athyros Vasilios

Atkinson David

Atkinson Greg

Atkinson Mark

Atnurkar Kishore

Atroshi Isam

Attai Dj

Aubin Henri-Jean

Auchincloss Amy

Audibert Martine

Auer Johann

Auf Rehab

August Furaha

Aune Dagfinn

Aung Eindra

Aung Koko

Aung Myo Nyein

Autier Philippe

Aveline C

Avent Minyon

Avery Amanda

Avery Ann

Avery Anthony

Avery Pj

Avidan Michael

Avidan Michael

Awad H

Awaisu Ahmed

Awasthi Shally

Awua Adolf Kofi

Ayala Marcelo

Ayar Hayat

Ayele Dg

Ayeni Olufemi

Ayeni Olufemi R

Ayis Salma

Aylin Paul

Ayling Kieran

Ayonrinde Oyekoya

Aypak Cenk

Azab Noha A

Azer Samy

Azim T

Aziz W

Azuma Kenichi

Baade Peter

Baalbergen Astrid

Baba Kyoko

Baba Masaru

Babatunde Opeyemi

Babson Ka

Babu Giridhara R

Baburdieri Sergio

Bach Bo

Bacharova L

Bachelot T

Bachkati Kristine

Bacon Paul 


\section{Badger $\mathrm{G}$ \\ Badia X}

Badri Shirinsadat

Badrick Ellena

Badrick Tony

Badry De

Bae Jong-Myon

Baekgaard Niels

Baena-Cañada José

Baessler Kaven

Bagby Michael

Bagga A

Baghaei Roshanak

Bagnasco Annamaria

Bahat G

Bahner David

Bahr Mark

Bahwere Paluku

Bai Ge

Baidal Jennifer Woo

Baig Faraz A

Bailey-Davis Lisa

Bailey A

Bailey Catherine

Bailey Douggl

Bailey Helen

Bailey Nick

Bailey Patricia

Bailey Sarah Lou

Bailey Tessa

Bain Re

Bain Stephen

Bainbridge Chris

Bainbridge Daniel

Bainbridge Kathleen

Bainbridge Lesley

Baines Cornelia

Baird Anne Marie

Baishnab E

Bakal Jeffrey

Baker Andrew

Baker Dian L

Baker Joshua

Baker Joshua F

Baker Kevin

Baker Md

Baker Nancy

Baker Paul

Baker Richard

Baker Ross

Bakhturidze George

Bakibinga Pauline

Bakker David

Bakker Marian

Balami Joyce

Balas Andrew

Baldari Carlo

Baldassarre Fulvia
Baldi Enrico

Baldwin Christine

Baldwin Constance

Baldwin David

Balevi Ben

Balhara Yatan

Balieva F

Ball Geoff

Ball Ian

Balmer Jt

Balogun Saliu

Balzer Laura

Banac Srdan

Banach Maciej

Banaszak-Holl Jane

Bandara Dinusha

Banerjee Amitava

Banerjee I

Banerjee Kushal

Banerjee Samprit

Baneshi Mohammad Reza

Banfai B

Bangalore Sripal

Bangpan Mukdarut

Banham David

Bankir Lise

Banks Jonathan

Banning $\mathrm{M}$

Bannister-Tyrrell Melanie

Banovic Marko

Bansal Aarti

Bansal Dipika

Banzi Rita

Bao Yp

Bao Yuqian

Baquero Daniel

Bar Zeev Yael

Bara David

Baradaran Hamid Reza

Barajas-Nava Leticia

Barallat Eva

Baranov Anton

Barba Carmen

Barbar Foss Kim

Barbara C

Barbati Giulia

Barber Matthew

Barber Philip

Barber S

Barbosa Ana

Barbouni Anastasia

Barcenas Ch

Barchi Leandro

Barco Stefano

Barcz Ewa

Bardou Marc

Barer David

Barger Brian
Barger Kathryn

Barger Steven

Barham-Brown Hannah

Barili Fabio

Barker Karen

Barker Mary

Barker Victoria

Barkham Michael

Barlow William

Barnard Kath

Barnes Deborah

Barnes Geoffrey

Barnes Hilary

Barnes Mary

Barnett Adrian

Barnett Carolina

Barnett Lauren

Barnett Michael

Barnett Paul

Barnett Stephen

Barnicot Kirsten

Barnoya Joaquin

Baron Emily

Baron Murray

Barone Mauro

Baroni Bruno

Barons Martine

Barrera Saldaña Hugo

Barreto Philipe

Barrett Drue

Barrett Peter

Barrett Sheila

Barros De Oliveira Sa

Michel Pompeu

Barsanti Sara

Barshes Nr

Barski D

Bart Gb

Barth Juergen

Bartlett Ah

Barton James C

Bartys Serena

Barzilay Joshua

Bas-Sarmiento P

Basak Ram

Bashford Tom

Baskar Rajamanickam

Basnet Sudha

Bass John L

Bassand Jean-Pierre

Bassano Carlo

Bassett-Gunter Rebecca

Bassetti Stefano

Basso Sm

Bassols Judit

Bast Lotus

Basta Giuseppina

Bastiampillai Tarun
Bastiani L

Baston Cameron

Bastuji-Garin Sylvie

Basu Anna

Basu Naren

Bataille Veronique

Bateman Nick

Bateni Hamid

Batista Ricardo

Batsis John A

Battaglia Salvatore

Battaloglu Bektas

Batterham Alan

Batterham Philip

Battersby Cheryl

Battista Ra

Batty David

Batura Neha

Bauer Brent

Bauer Jan

Bauer Stuart

Baulig Christine

Baum Michael

Bauman Adrian

Baumeister David

Baussano Iacopo

Bauwens Jorgen

Bavendiek Udo

Baxi Shrujal

Baxter Nancy

Baxter Ruth

Bayarsaikhan Dorjsuren

Baydemir Canan

Baye K

Bayer Tony

Baylan Satu

Bayley Julie

Bayman Emine

Baynes Habtamu

Bayram A

Baysari Mt

Bazzano Alessandra

Beadsworth Mb

Beal Matt

Beale Anna

Beales Darren

Beard Renee

Bearden David R

Beaton Andrea

Beattie Bryan

Beaudart Charlotte

Beaudoin Francesca L

Beaulieu

Marie-Dominique

Beaumont J

Beavers Dp

Bebell Lm

Becher Jg 
Beck Ben

Becker Annette

Becker Christian

Becker Christine

Becker Soeren

Becker Torben

Beckman Anders

Beckman Linda

Beckman Thomas

Beddoe Liz

Bedi S

Beeken Rebecca

Beerman Stephen

Beets Michael

Beg Mirza

Begg Alan

Beghi Massimiliano

Behanova Martina

Behera Arunanshu

Behmanesh Fereshteh

Behrens Thomas

Beige Joachim

Beijamini F

Beike Dr

Beilby Justin

Beiles Charles

Beitsch Lm

Bekhor Philip

Beksinska Mags

Belfiore Maria Paola

Beliaev Andrei

Bell Colin

Bell James

Bell Lucy

Bellamy Gr

Bellan Mattia

Belley-Coté E

Bellisola Giuseppe

Bellolio M Fernanda

Bellón Juan A

Belloti João

Bellou Abdelouahab

Belvederi Martino

Bem Danai

Bemben Debra

Ben-Moshe Ros

Ben-Shlomo Yoav

Ben-Shoshan Jeremy

Ben-Yakov Maxim

Benagiano G

Bénard-Laribière Anne

Benardot Dan

Benedetti Andrea

Bener A

Benhalima Katrien

Benhamou Dan

Benhamou Pierre-Yves

Benjamin Neelon Sara E
Benkhadra Mehdi

Benn Marianne

Bennasar-Veny Miquel

Bennett Derrick

Bennett Kathleen

Bennett Sophie

Bennie Marion

Bennis Issam

Benson John

Bentley Suzanne

Benton Sherry

Benzaken Adele

Benzies Karen M

Benzinger $\mathrm{P}$

Benzo Roberto

Beran R G

Berecki-Gisolf Janneke

Berecki-Gisolf Janneke

Beretta-Piccoli Benedetta

Berezin A E

Berezuk Courtney

Bergasa Nora

Bergeron Cristine

Bergese Sergio

Bergh S

Berghold Andrea

Berglas Nancy

Berild D

Berkelhamer Sara

Berker B

Berkhof Johanes

Berkhout Christophe

Berkley James

Berkowitz Seth A

Berlin Claudia

Berlin I

Berlin Jesse

Bernabe-Ortiz Antonio

Bernal William

Bernardes Vanessa

Bernardo Wanderley

Berni Canani Roberto

Bernstein Rebecca

Bernstrøm Vh

Berntorp Kerstin

Berntsen Gro

Berr Claudine

Berrett Andrew

Berrouiguet Sofian

Berruti Alfredo

Berry Donna

Berry Jay

Berry Jl

Bertelsen Jannik

Bertolini Guido

Berton Giuseppe

Berube Melanie

Besen Bruno
Beshai Shadi

Best Catherine

Best Lawrence

Beswick Andrew

Bethge M

Betrá n Ana Pilar

Bettelli Gabriella

Bettencourt-Silva Joao H

Betz Joshua

Betz Marian E

Bevacqua Brian

Bevan Gwyn

Bevc Sebastjan

Beverly Elizabeth

Bhagwanjee S

Bhan Gautam

Bhana Arvin

Bhandari Mohit

Bhandari Sunil

Bhangu Jaspreet

Bharateesh Jayanna

Bhat Abdul Rashid

Bhatia Jatinder

Bhattacharya Sohinee

Bhattacharyya I

Bhattacharyya Sagnik

Bhattacharyya Sanjoy

Kumar

Bhaumik Soumyadeep

Bhopal Raj

Bhowmik Bishwajit

Bhuiyan Mirza

Bhutta Zulfiqar

Bhuyan Basant Kumar

Bian Zhaoxiang

Bianco John

Bice Thomas

Bickley Harriet

Biddle M

Bidonde Julia

Biele Guido

Bielory Leonard

Bienvenu O J

Biffi A

Bihari Shailesh

Bihorac Azra

Bilgin G

Billett Stephen

Billings Ft

Billings Jenny

Billot Laurent

Bilo Henk

Bina Rena

Bind Marie-Abele

Bion Julian

Biondi A

Biondi Zoccai Giuseppe

Biondi-Zoccai Giuseppe
Biram Richard

Birch Stephen

Bird Geoff

Bird Julia

Bird Marie-Louise

Bird Sheila

Birenbaum-Carmeli

Daphna

Birgersson Ulrik

Birlutiu Rm

Birnbach David

Birnie Kate

Biros Michelle

Birrell Paul

Bisanzo Mark

Bischof Gallus

Bisgaard Pedersen

Anne-Marie

Bishop-Bailey D

Bishop Andrea

Bishop Felicity

Bishop Lisa

Bissessor Melanie

Bisset Leanne

Biswas Arijit

Bittencourt Marcio

Bittoni Marisa A

Bitzan Martin

Bizzarro Matthew

Bjelland Elisabeth Krefting

Bjerkreim Anna

Bjerregaard Anne-Louise

Bjerrum Lars

Bjørgaas Marit

Black Corrinda

Black Rj

Blackburn Carolyn

Blackwell Caroline

Blæhr Emely Ek

Blair Aaron

Blake Dj

Blake Miranda

Blakely Brette

Blakeman Tom

Blalock Travis

Blanchard Janice

Bland Jeremy

Bland Martin

Blandino Giovanni

Blane David

Blankers Matthijs

Blanpain Cedric

Blanquisco Lr

Błaszczyk B

Blazeby Jane

Bleakley Alan

Blecher Evan $\mathrm{H}$

Bleidorn J 
Bleijenberg Nienke

Blendon Robert

Blettner Maria

Bletzer Keith

Blickem C

Bliddal Mette

Blizzard Christopher Leigh

Bloch Michael H

Block Geoff

Blockeel Christophe

Blockley Kelly

Blome Christine

Blomgren Jenni

Blondeel Karel

Bloomgarden Zachary

Blue Cm

Blum Jennifer

Blumenthal David

Boatin Adeline

Boaz Annette

Boaz Mona

Bobay Kathleen

Bobevski Irene

Bochenek Tomasz

Boddy Ap

Boden Joseph

Bodilsen Jacob

Boe Shaun G

Boehm Katja

Boehmer Kasey R

Boehnke Jan Rasmus

Boeldt Debra

Boender T Sonia

Boerma Marjan

Boers M

Boersma D

Boetker Morten

Boffano Paolo

Boffi Roberto

Bogani G

Bogdanici Camelia

Bohannon Richard

Boissy Adrienne

Boland Michael

Bolen Jen

Bolgla Lori

Bollag Ueli

Bolland Mark

Bollig Goerg

Boman Kurt

Bommer Christian

Bonander Carl

Bonati Maurizio

Bonati Maurizio

Bond Christine

Bond Malcolm

Bondevik Gunnar
Bonello Laurent

Bonet-Carne Elisenda

Bonfiglioli C

Bonham Maxine

Bonn Stephanie

Bonnaire Céline

Bonnett L

Bonney E

Bonomi Stefano

Bonzel Tassilo

Bonzini Matteo

Booker C

Booker Matthew

Boomsma Martijn

Boon Michelle

Boonstra Philip

Boot Cecile R L

Boote Jonathan

Booth Alison

Booth Andrew

Booth Vl

Borch C

Borczuk Pierre

Bordini B

Borgquist Lars

Borgsteede $\mathrm{Sd}$

Boriboonhirunsarn

Dittakarn

Borja Caroline

Borland Ron

Born Karen

Bosanquet David

Bosanquet Nick

Bosch Tyler A

Bostwick Dg

Bosu William K

Botelho R

Bots Michael

Bottcher Bettina

Bottle Alex

Bouchard Phillipe

Bouldin Erin

Boulesteix Anne-Laure

Boullata Joseph

Bouman Chantal

Bourgeault Ivy

Bourgeois Denis M

Bourgeois Florence

Bourke L

Bouter Lex

Boutron Isabelle

Bouwman Renée

Bouwmeester Carla

Bouzat Pierre

Bouziane Amal

Bower Peter

Bowers K

Bowles Erin
Bowman John D

Bowman Maria

Bowry Ashna

Boyanova L

Boyce Robert

Boyle Adrian

Boyle Coleen

Boyle Elizabeth

Boyle Elizabeth A

Boyle Em

Boyles Tom H

Brace-Mcdonnell

Samantha

Brach Condy

Bradburn Mike

Bradbury-Jones C

Bradley Claire

Bradley Elizabeth

Bradley Marie

Bradley Patrick

Bradshaw Pamela

Brådvik Louise

Brady Ellen

Brady Paul

Braeckman Lutgart

Bragazzi Nicola

Bragg Fiona

Brailsford Mandy

Brainin Michael

Brämberg Eb

Bramley George

Brand-Miller Jennie

Brand Serge

Brandenburg Vincent

Brandi Maria

Brandt Nicole

Brannsether Bente

Brantsaeter Anne Lise

Braschinsky Mark

Brassard Paul

Bratlid Dag

Bratt Ola

Brattebø Guttorm

Braun J

Braun Kathryn L

Braun Tom

Braund Rhiannon

Braunstahl G

Bray Issy

Bray Janet

Breban Maxime

Breckwoldt Jan

Breik Omar

Bremner Stephen

Brender Jean

Brener Michael

Brennan Nicola

Brennan Paul
Brentnall Adam

Bressan Silvia

Bressi Cinzia

Bressington Daniel

Brett Tom

Brettschneider Christian

Brevik Harald

Brewer Andrew

Brewster David

Bridges Diane

Briel Matthias

Brier Michael

Briggs Adam

Bright Felicity

Bright Melissa A

Bright Tess

Brighton Lisa

Brignardello-Petersen

Romina

Brignell Amanda

Briley A

Brillanti Stefano

Brimblecombe Julie

Brimblecombe Neil

Bringolf-Isler B

Brinkhof Martin

Brinkman David

Brinn Malcolm

Brites Carlos

Britton P N

Brochhausen Mathias

Brodlie Malcolm

Brodtmann Amy

Broekstra Dc

Bromfield Sg

Bromme Rainer

Brønnum-Hansen Henrik

Bronstein Janet

Bronstein Jm

Brook Gary

Brooke Benjamin

Broom Jennifer

Brophy James

Bross Donald

Brothers Td

Brouwer Miranda

Brown Jr Raeford E

Brown Ca

Brown Cl

Brown David

Brown Elise

Brown Heather

Brown James

Brown Jc

Brown Jeremy

Brown K

Brown Rs

Brown Siobhan 
Brownie Sm

Bruce Julie

Bruckner Tim-Allen

Brugaletta $S$

Brüggmann Dörthe

Bruha Radan

Bruins Slot Karsten

Bruinvels Georgie

Brumfitt Shelagh

Brun Jean-Frédéric

Brunak Søren

Brunetti Natale

Brunetti Natale Daniele

Brunham Lr

Brunner Dr Iris Charlotte

Bruno $G$

Brunton Margaret

Brusasco Vito

Brusselaers Nele

Brussoni Mariana

Bryant Maria

Bryant-Lukosius Denise

Bryson Gregory L

Brzoska Patrick

Buccheri Dario

Buchanan Helen

Buchvold Hogne

Buckley Helen

Buckley Nicholas

Buckley Nicholas

Buckley Rachel

Budd Simon

Budde Klemens

Buecking B

Bueno-Marí Rubén

Buffarini Romina

Buffart Lm

Bugiardini Raffaele

Buha Aleksandra

Buiting Hilde

Buja Alessandra

Bujold Emmanuel

Bükki J

Buldt Ak

Buller David B

Bump Gm

Bunce Catey

Bundhun Pravesh

Buntinx Frank

Burchell A

Burchett Helen

Burchill Cn

Burdorf Alex

Burford B

Burger $\mathrm{H}$

Burgess J

Burgess Marion

Burgner David
Burgosvargas Rubé n

Burhan Hasan

Burke Eilish

Burkhart Gregor

Burkill Sarah

Burkom Howard

Burn Anne-Marie

Burney Peter

Burns Pippa

Burns Ryan

Burns Tania

Burren Christine

Burroughs Pena Melissa

Burrows Raquel

Burstyn Igor

Burt Morton

Burtin Chris

Burton Christopher

Burton $\mathrm{Cl}$

Burton Claire

Burton Elissa

Burton Kim

Burton Maria

Burtscher Martin

Busari Jamiu

Busby John

Buschmann C T

Bush Shirley H

Busija Lucy

Busnelli Andrea

Bustamante Alejandro

Butcher Anna

Butler C

Butler Mary

Butt Thomas

Butts Charles

Buykx Penelope

Buys R

Byakodi Sanjay

Byler C

Byles Julie

Byrne Anthony

Byrne Hugh

Byron M

Byszewski Anna

Cable Noriko

Cabrera-Barona Pablo

Cabrera Irene

Cacciatore Francesco

Cachat Francois

Caciula Manuela

Cadilhac Dominique

Caetano M

Cahill David

Cai Gaojun

Cai Guoxiang

Cai T

Cai Yutong Samuel
Cailhol Johann

Caille Agnès

Cairns Melinda

Calambur Narsimhan

Calan Mehmet

Calandre Elena

Calanzani Natalia

Calati Raffaella

Caldas Jamil Pedro

Caldeira Daniel

Calder P

Calderón De La Barca Am

Calderón-Larrañaga

Amaia

Caldwell Deborah

Calear Alison L

Calixte Rose

Caljouw Monique A A

Callaghan-Koru Jennifer A

Callaghan Charlotte

Callard Felicity

Callas Peter

Calvert James

Calvo Gonzalo Segrelles

Camacho Elizabeth

Camargo Constanza

Camdeviren Ankarali

Handan

Cameron Noël

Camilo Millene R

Camp Charlotte

Campanella Fabio

Campanile Fabio Cesare

Campanini Marcela

Campbell Jared M

Campbell B

Campbell David

Campbell Fiona

Campbell Helen

Campbell John

Campbell Kristen

Campbell Mhairi

Campbell Michael

Campbell Narelle

Campbell Noll

Campbell Paul

Campbell Tavis

Camus Enrique

Canabarro Antonio

Canan Chelsea

Canaway Alastair

Canizares Mayilee

Cannegieter Suzanne

Cannon Mary

Canpolat Uğur

Cantarero-Arévalo

Lourdes

Canty David
Cao Guangwen

Cao Yang

Capan Muge

Cape John

Caplan Gideon

Caplan Louis

Capstick Robert

Capuano Annalisa

Caranti Danielle

Carbone Elena T

Carbone Stefano

Cardenas Jessica

Cardenas Victor

Cardiff Lynda

Cardoso Éder

Cardoso Marly

Carey Iain

Caricato Anselmo

Carinci Fabrizio

Carl Rebecca L

Carlesso Lisa

Carlin Emma

Carling Malin

Carlisle Karen

Carlo Waldemar

Carlsson A C

Carlsson Tommy

Carlton Edward

Carmichael Amtul

Carnes Dawn

Caron Maryse

Carr David

Carr Eloise

Carr Zhanat

Carrà G

Carradice D

Carrier Judith

Carrière Gm

Carrillo-Larco Rodrigo

Carroll Christopher

Carson-Chahhoud Kristin $\mathrm{V}$

Carson Dean

Carson Wf

Carter B

Carter Ben

Carter Marissa

Carter Nancy

Carter Pam

Carter Stacy

Carter Tonia

Carton Matthieu

Cartwright Rufus

Caruso Rosario

Carvajal Alfonso

Carvalho Dos Santos

Jussara

Carwile Jenny 
Casalino Enrique

Casamassimi Amelia

Casanova Ludovic

Casas Maribel

Casciaro Sergio

Case Philippa

Caselli Desiree

Casellini Carolina

Casey Anna

Casey Anne-Nicole

Cassady Jerrell

Cassidy-Bushrow Andrea

Cassidy Sophie

Castagna L

Castañeda Santos

Castel Liana

Castelein René

Castelli Adriana

Castellini Greta

Castelnuovo Barbara

Castiglione G

Castro-Diehl Cecilia

Castro-Sanchez Adelaida $\mathrm{M}$

Castro-Sánchez Enrique

Catalá-López Ferrán

Catalan Jose

Catalano A

Catalano Antonino

Catchpoole Daniel

Cates Joan

Cates Jordan E

Cather Corinne

Catherine M Roe

Cations Monica

Catroppa Cathy

Cattano Davide

Caughey Ab

Causer Louise

Cavaco Afonso Miguel

Cavalheri V

Cavallari Michele

Cavalot F

Cavarretta Elena

Cavassan De Camargo Caio

Cavers Debbie

Cavicchi Caterina

Cavusoglu Yusuf Hakan

Cayetano Shari Messinger

Cazzola Mario

Cecilio-Fernandes Dario

Celebi Z K

Celentano Valerio

Celic Robert

Celik Mustafa

Cellini Nicola

Cerrittelli Francesco
Cesana Bruno Mario

Ceschi Alessandro

Cevoli S

Chaabna Karima

Chabowski Mariusz

Chabriat $\mathrm{H}$

Chachou Martel

Chacón-Moscoso Salvador

Chadborn Neil

Chadha Nisha

Chadha Shelly

Chadwick David

Chai Qianyun

Chaiton Michael

Chakrabarty Samit

Chalmers Leanne

Chambarae Keneeth

Chamberlain Stephanie

Chambers Charlotte

Chambers Georgina

Champenois Karen

Champredon David

Chan Caryn

Chan Christian

Chan Daniel

Chan David S Y

Chan Gary

Chan Helen

Chan Hung-Yu

Chan Jason

Chan Ming-Ka

Chan Ngai-Yin

Chan Yoke Mun

Chan Zenobia

Chanani Nikhil

Chandola Tarani

Chanel Olivier

Chang Alex

Chang Bei-Hung

Chang Chun

Chang Hsing-Yi

Chang Jeffrey

Chang Jung-Chen

Chang K

Chang Ku-Chow

Chang Larry

Chang Po-Yin

Chao Chia-Ter

Chao Christopher

Chaparro-Narváez Pablo

Chapman Dk

Chapman Stephen

Chappell Dr Lucy

Chappell Francesca

Chapple Cathy

Charette Yanick

Charfi Rim

Charles Joanna
Charlesworth Georgina

Charlson Fiona

Charlton Karen

Charman Tony

Charran Ak

Chase Janet

Chattat Rabih

Chatterjee Helen

Chatterjee Sudipto

Chatterji Somnath

Chatterton Mary Lou

Chaturvedi Sarika

Chatzimavroudis Grigoris

Chatzizisis Ys

Chaudhary Neeraj

Chauduri P

Chaumba Josphine

Chaurasia Alok Ranjan

Chauvet-Gelinier

Jean-Christophe

Chavarro Jorge

Chazapis M

Cheah Wee Kooi

Checherita Ionel

Alexandru

Checkley William

Cheen Mcvin Hua Heng

Chemweno Peter

Chen Hui

Chen Ac

Chen Bradley

Chen Chung-Yu

Chen Cynthia

Chen Fj

Chen George

Chen Gong

Chen Han-Yang

Chen Jen-Hao

Chen Jonathan

Chen Juan

Chen Kathryn T

Chen Lee-Lynn

Chen Lee-May

Chen Liang-Kung

Chen Mingsheng

Chen Ping

Chen Sc

Chen Shih-Ann

Chen Shihui

Chen T

Chen Ta-Liang

Chen Wanqing

Chen Wei

Chen Weihong

Chen Wen

Chen Xiangmei

Chen Yajun

Chen Yen-Fu
Chen Yuchen

Cheng Allen

Cheng Chih-Hsiu

Cheng Hung-Yuan

Cheng Jing

Cheng Kai-Wen

Cheng Li

Cheng Yu

Chenot J -F

Cheriyan Thomas

Chernesky Max

Chersich Matthew

Chervenak Frank

Cheslack-Postava Keely

Chesser Tim

Cheung As

Cheung Bernard

Cheung Derek

Cheung Kei Long

Cheung Kin

Cheung R

Cheung Teris

Cheungpasitporn Wisit

Chew-Harris Janice

Chew Kah

Chew Lita

Cheyney Melissa

Chhapola Viswas

Chhatre Sumedha

Chi Ching-Chi

Chi Ly

Chia Audrey

Chia Karen

Chiao-Hsiung Chuang

Chiarotto Alessandro

Chiba R

Chibanda D

Chico R Matthew

Childs Emma

Chin Chloe

Chin David L

Ching Siew Mooi

Chiodini I

Chiolero Arnaud

Chipps Jennifer

Chitekwe Stanley

Chiu Chong-Chi

Chiu Hc

Chiumello D

Chiumento Anna

Chivers Corey

Chivese Tawanda

Chiwanga Faraja

Chmiel C

Cho Chang Min

Cho Hangjoo

Cho Hyunkag

Chodick Gabriel 
Choi Seung-Hoon

Choi Eun Hwa

Choi Jin-Ho

Choi Kelvin

Choi Kui Son

Choi Leslie

Choi Se

Choi Seong-Woo

Choi Seung Hee

Choi Stephen

Chojenta Catherine

Chokan Mohammad

Chong Vui Heng

Choo Yao Mun

Choonara Imti

Choudhary $\mathrm{P}$

Choukem Simeon-Pierre

Chow Eric

Chowdhury Tumul

Chrisinger Bw

Christensen Karl

Christiansen $\mathrm{Ob}$

Christie Catherine

Christie Deborah

Christie Janice

Christodoulou Nikos

Chu Cordia

Chu Nain-Feng

Chu Nain-Feng

Chua Yp

Chuang Elizabeth

Chuang Hung-Yi

Chuenjitwongsa Supachai

Chun Byung

Chung Chun Kee

Chung Ka-Fai

Chung Kuo-Piao

Chung Raymond

Chung Sharon

Chung Sheng-Chia

Chung Tammy

Churchill Larry

Churov Alexey

Ciaccio Marcello

Ciaramella A

Ciarleglio Adam

Cicchino Jb

Cicero Arrigo

Cicero Arrigo Francesco

Giuseppe

Cicolini Giancarlo

Ciconte Giuseppe

Cífková Renata

Cihoric Nikola

Cioni Giovanni

Cipriani Andrea

Cirillo P

Cittadini Antonio
Claassens M

Claes Jomme

Claggett Brian

Clark Christopher

Clark David

Clark Emma

Clark Helen

Clark Jeffrey

Clark Vanessa

Clarke Caroline

Clarke Charlotte

Clarke Damian

Clarke E

Clarke Joanne

Clarke Katherine

Clary Christelle

Clase Catherine

Clausen Thomas

Clausen Tine

Clauss Elisa

Clayton Gemma

Cleaver Karen

Cleland Jennifer

Cleland Verity

Clemens $\mathrm{T}$

Clemens Tessa

Clement Fiona

Clement Nicholas

Cleverley Kristin

Clifford Gary

Clissett Philip

Cloninger C Robert

Cloutier Lyne

Clucas Claudine

Clyne Barbara

Coady Sean A

Coan Pm

Coates Alison

Coates Dominiek

Coates Laura

Cobham Vanessa

Cobo Erik

Coburn Mark

Cochrane Lynda

Cochrane Tom

Cockburn William

Cockcroft Anne

Coelho Ana

Coetzee Bronwyne

Coetzee Kevin

Coghe G

Cohen-Solal Martine

Cohen Cheryl

Cohen Elizabeth

Cohen Jm

Cohen Joel

Cohen Jonathan

Cohen Steven
Cohn E

Col Nananda

Colantonio Ld

Colantuoni E

Colborn Kathryn

Colbourn Timothy

Cole Tim

Coll-Planas Laura

Colleti Jose

Collett Dave

Collett Johnny

Collie Alex

Collier A

Collier Fiona

Colligan Erin

Collins Jeffery

Collinson Paul

Collinsworth Ashley W

Collste Olov

Colman Ian

Colombo Cinzia

Colquhoun David

Colubri Andres

Colver Allan

Comandone Alessandro

Comans Tracy

Comer Christine

Compernolle Sofie

Conaghan Philip

Conboy Lisa

Conde-Taboada A

Condon Louise

Congdon Peter

Conigrave Kate

Conklin Annalijn

Conner Mark

Conner Tamlin

Conroy Simon

Consedine Nathan

Consoli Arturo

Constantinou Panayotis

Contandriopoulos Damien

Conte Antonio

Contento I

Conti Go

Conti Sarà

Contiero Paolo

Contreras-Urbina Manuel

Conway-Morris Andrew

Conwell Yeates

Cook Ad

Cook Andrew

Cook Brian J

Cook Penny

Cook Stephane

Cook Tim

Cooke Debbie

Cooke Richard
Cookson Tara

Coolsen Marielle

Coombes Lucy

Coon Cheryl

Cooney Laura

Cooper Jackie

Cooper Jamie

Cooper K

Cooper Maria

Cooper Richard

Cooper Richard

Cooper Rosie

Cooper Sally-Ann

Cooper Simon

Cooper Tess

Coote Philip

Copas Andrew

Cope Anwen

Coppo Alessandro

Coppola Adriana

Coquart Jb

Coquerel Antoine

Corbett Mark

Corcia Philippe

Corcoran Michael

Cordeiro E

Cordoba Gloria

Corish Clare

Corlin Laura

Cormier Stephanie

Cornall Alyssa

Cornelis Tom

Cornette Jérôme

Corniola M-V

Cornish Rosie

Corradi Massimo

Correa-De-Araujo1 Rosaly

Corrêa-Faria P

Correia De Sousa Jaime

Corrie Philippa

Corso Christopher

Corson Stephen

Corsonello Andrea

Cort Malcolm A

Cortegiani Andrea

Cortes Castell Ernesto

Cortes-Sanabria Laura

Cortez-Pinto Helena

Cortina Borja Mario

Cosgrove Joe

Cosma Dan

Cosmi Benilde

Cosson Emmanuel

Costagliola Ciro

Costanian Christy

Cöster Maria

Cotrim Teresa

Cotterill Sarah 


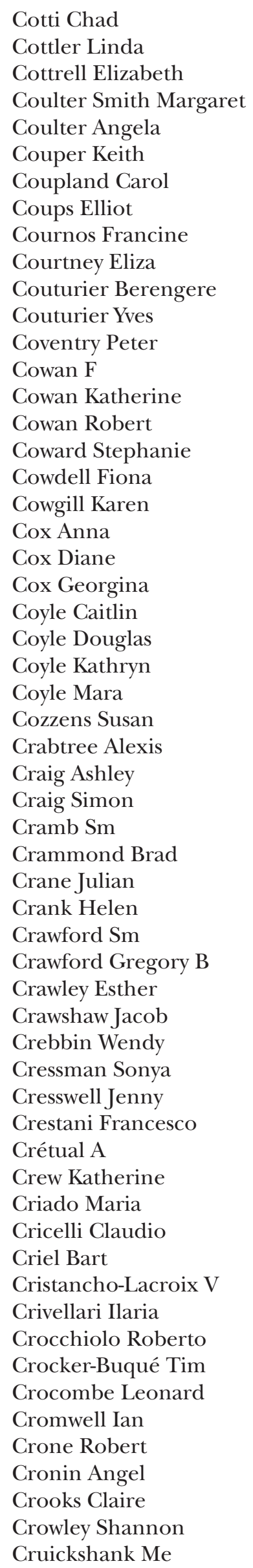

Cryder Brian

Cubała Wj

Cucchiaro Giovanni

Cuenca-Estrella Manuel

Cui Jie

Cui Y-M

Cui Yang

Cuijpers Pim

Culig J

Culpin I

Cummings Elizabeth

Cummings Michael

Cummins Carole

Cundiff Jenny

Cunningham Antony

Cunningham John

Cunnington David

Cuomo Barbara

Cuppari Lea

Curcio Kristin R

Curran Vernon

Currie Cheryl L

Currie Craig

Currie Sinead

Curtis Kristina

Cuschieri Kate

Cuschieri Sarah

Custers Roel

Cusworth Walker S

Cutland Clare

Czosnyka Marek

D'amico Francesco

D'arcy Carl

D'ascenzo Fabrizio

D'auria Enza

D'elia L

D'oliveira Af

D'silva Adrijana

Da Costa Fa

Da Silva Andréa

Da Silva Inácio

Da Silva Marisa

Dacks Penny

Dadi AF

Dadu Rt

Daga Sunil

Dagenais Christian

Dagfinrud Hanne

Dagliati Arianna

Daglish Mark

Dagnelie Gislin

Dagogo-Jack Sam

Daher Ghassan

Dahlen Hannah

Dahlmann-Noor Annegret

Dahly Darren

Dahm Philipp
Dahm Christina

Dai Zhiyuan

Daiber Andreas

Daidone $\mathrm{Mg}$

Dainichi Teruki

Dake F

Daker-White Gavin

Dal Re Rafael

Dal-Ré Rafael

Dalal Hm

Dalbo Vincent

Daley Amanda

Dalhoff Kim

Dalkin Sonia

Dall Philippa

Dall Timothy

Dall'ora Chiara

Dallat Mary

Dalle Grave Riccardo

Dalton Alice

Daly Chris D

Daly Lisa M

Daly Rachel

Dam Rob

Damarell Ra

Dami Fabrice

Damiani E

Danaei Goodarz

Danauer Brigitta

Dandona Rakhi

Daneman Nick

Danese Mark

Daniels Jo

Daniels Robert D

Danve Abhijeet

Daray Federico

Daraz Lubna

Darby Sarah

Darker Catherine

Darlow Ben

Darmon M

Darnai G

Darney Blair

Darton Thomas

Darzi Ara

Das-Munshi Jayati

Das Bhudev

Das Bidhan Kanti

Das Dilip Kumar

Das Jai K

Das Rashmi

Datta Ak

Datta Deepankar

Datta Yvonne H

Dauphinee Dale

Davenport Clare

Davey Mary-Ann

David Jean-Stéphane
David Matthias

David Michael

Davidson Andrew

Davies-Tuck Miranda

Davies Christina

Davies Freya

Davies Mark

Davies Peter

Davies Ronnie

Davies SJ

Davis $\mathrm{Sr}$

Davison Karen

Dawda Paresh

Dawe David

Dawe Russell

Dawes Martin

Dawson Angela

Dawson Nancy L

Dawson Pamela

Dawson Robert

Dawson Suzanne

Day Carolyn

Day Ed

Day Rosie

Day Te

Dayan Natalie

De Juriena

De Almeida Jr Hiram

De Borst Gert-Jan

De Boysson Hubert

De Briyne N

De Broe Marc

De Bruin Eduard

De Bruin M

De Bruyne Mieke

De Carlo Marco

De Carvalho Vidigal F

De Costa Caroline

De Courten Barbora

De Falco Maria

De Frutos Fernando

De Giorgi A

De Girolamo Giovanni

De Gonzalo-Calvo David

De Graaff Barbara

De Groef An

De Groot Bas

De Groot J

De Guzman Raegel

De Jager Elzerie

De Jong Gideon

De Jong Ka

De Jongh M

De Jongh Renate

De Klerk Nicholas

De Kreutzenberg Saula

De Kuijper G

De Kunder Sl 
De La Rosa Diaz Irene

De La Rosette Jean

De La Torre Gg

De Las Peñas Ramon

De Lauretis A

De Lorenzo A

De Mestral Charles

De Miguel Manuel

De Nazelle Audrey

De Oliveira Beck Roberto

Miquelino

De Rooij Belle

De Ruijter Dennis

De Santo Luca

De Sensi Francesco

De Silva Da

De Siqueira Galil Ag

De Socio Giuseppe

Vittorio

De Souza Dziedzom K

De Souza Russell

De Stampa M

De Vito Andrea

De Voursney David

De Vries Eline

De Vries Esther

De Vries Fleur

De Vries NM

De Wildt Saskia

De Windt Tommy

De Zambotti M

De Sukanya

Deady Mark

Deakin Trudi

Dealy Bern

Dean Benjamin

Dean Elizabeth

Dean Elizabeth

Dean Natalie

Dean Sarah

Dean Sue

Deane Adam

Deane Katherine

Deans Kj

Debono Miguel

Debray Thomas

Dechartres A

Dechristopher Lr

Decraemer Marieke

Decullier Evelyne

Dede Ad

Dedefo M

Deeks Shelley

Deguen Severine

Dehbi Hakim-Moulay

Dehmer E

Dehon Erin

Deilkås Ellen
Dekhtyar Serhiy

Dekker Joost

Del Carpio Munoz Freddy

Del Fiol Guilherme

Del Mar Chris

Del Re Ac

Del Rio-Martinez Pilar

Delanaye Pierre

Delaney Kathleen R

Delbaere M

Deleuran T

Delfabbro Paul

Delgado Mk

Delisle Jean-Sébastien

Dellamonica Jean

Dellavalle Robert

Dello Strologo Luca

Delnord Marie

Deluca Paolo

Deluca Stephanie

Demartines Nicolas

Dembowska-Baginska Bozenna

Demczuk Walter H B

Demmelmair Hans

Demou Evangelia

Demoule Alexandre

Dencker Anna

Deng Yanhong

Denney Elaine

Dennis Brittany

Dennison Meg

Denny Simon

Denoeud-Ndam Lise

Dentali Francesco

Denton E

Depuydt Pieter

Deressa T

Deri Armstrong Catherine

Deribe Kebede

Deright Jonathan

Derksen Frans

Derman Sonja

Dersch Rick

Derwinger Kristoffer

Des Jarlais Don

Desai Jagdish

Desai Sapna

Desan Paul H

Desantis C

Desantis Sm

Desborough Jane

Descallar Joseph

Descatha Alexis

Deshpande Aniruddh V

Deshpande Anshula

Desmet A

Desrosiers Martin
Detanti Antonio

Detmar Symone

Deuter Kate

Devaney John

Deveza Leticia

Devilliers Anniza

Devine Katie

Devonald Mark A J

Dewan Michael

Dewan Neha

Dewaure Chiara

Dewey Marc

Deyle Gail D

Dhaini Hassan

Dhaliwal Satvinder

Dhandapani

Sivashanmugam

Dhargave Pradnya

Dharmananda Subhuti

Dharmarajan Kumar

Dhelaria RK

Dhillon Mandeep

Dhippayom Teerapon

Di Castelnuovo Augusto

Di Domenicantonio

Riccardo

Di Giacomo Patrizia

Di Giorgio Laura

Di Gravio Chiara

Di Lullo Luca

Di Martino Mirko

Di Massimo Dino

Di Napoli Mario

Di Ruggiero Erica

Di Saverio Salomone

Di Somma Salvatore

Di Jl

Diaby V

Diallo Karidia

Diamanti-Kandarakis

Evanthia

Diament D

Dias Sofia

Díaz J

Díaz A

Diaz Francisco

Dibley Lesley

Dickens Andy

Dickersin Kay

Dickert Nw

Dickinson Helen

Dickman Samuel

Dickson-Gomez J

Dieli-Conwright C

Diem Gunter

Diener M K

Dieterich M

Diez Julia
Dijkers Marcel

Dijkgraaf Marcel

Dijkstra A

Dilla Tatiana

Dilles Tinnes

Dillier N

Dilokthornsakul Piyameth

Dimairo Munyaradzi

Dimario Simona

Dimick Justin

Ding X Q

Ding Ying

Dinh Dzung

Dinneen Sean

Dinos $S$

Direito Artur

Dirienzo Gregory

Dirksen Carmen

Dirlikov Emilio

Dismuke Clara Elizabeth

Ditlopo Prudence

Divito Sherrie

Dixon Padraig

Djaiani George

Djossou F

Djulbegovic Benjamin

Do Nascimento Marreiro D

Do Albert

Do Elizabeth K

Do Huyen

Do Jae

Do La

Dobb Geoffrey

Dobbinson Suzanne

Dobbs Debra

Dobkin Bruce

Doble Brett

Dobre Mirela

Dobruch Jakob

Dobscha Sk

Doctor Hv

Dodd Rachael

Dodd Susanna

Dodek Peter

Doerken Sam

Doh Young

Doherty Tanya

Dohle Gr

Doi Suhail

Doig Gordon

Doku David

Dolan Eamon

Dominguez Angela

Donal Erwan

Donaldson Alex

Donate-Martinez

Ascension 
Donati Mauro

Donchin Yoel

Donegan Katherine

Dong Xiuwen Sue

Dong Min

Dong Wei

Dong Yiran

Donker T

Donkor Noble

Donnelly Elizabeth

Donnelly S

Donnelly Tam Truong

Donner Claudio F

Doolan-Noble F

Dooms Marc

Doos Lucy

Doran Kelly M

Dori Guy

Dorin Richard I

Dörks M

Dorling Danny

Dornan Timothy

Dossou Jean-Paul

Dotchin Catherine L

Doty Richard

Doubova Svetlana

Doucette William R

Douglas Clint

Douglas Mbuyiselo

Douglas Vanja

Doust Jenny

Dovey Terence

Dowell Jon

Downer Brian

Downey Candice

Downie Aron

Downie Laura

Downie Michelle

Downing A

Downing Karrie

Downing Kl

Dowrick Cf

Dowrick Christopher

Dowthwaite Jodi

Doyle Colleen

Doyle Orla

Drabo Emmanuel

Dragano Nico

Drake Bettina

Dray Julia

Dreesman J

Dregan Alexandru

Dreiher Jacob

Dres Martin

Dresden Scott

Dressel Ralf

Drevenhorn Eva

Dreyer Rachel
Driessnack Martha

Drucker Aaron

Druetz T

Du Prel Jean-Baptist

Du Huaidong

Du Juan

Du Shufa

Dualé Christian

Duan Jie

Dubey Ashutosh

Dubois Jean-Daniel

Dubowitz Howard

Dubrey Simon

Duckett Stephen

Duckworth Andrew

Dufouil Carole

Duggleby Wendy

Duits Aj

Duman Fulya

Dumuid D

Dunbar David

Dunbar Nm

Dunbar R

Duncan Mitch

Duncan Steven

Duncan W

Dunn Adam

Dunn Carolyn

Dunn Kelly

Dunn Michael S

Dunn Sheila

Duong Andrew

Dupont Alain G

Durand-Zaleski Isabelle

Durdu Murat

Durmic Tijana

Dussault Gilles

Dutil J

Dutkowsk Phillip

Dutra Lauren

Dutra Sunny

Dutta Dipankar

Dwivedi Alok

Dyda A

Dykes Patricia

Dyson Simon

Dzhambov Angel

Dzikiewicz-Krawczyk A

Eadie Mervyn

Eastgate G

Eastwood Alison

Eaton Simon

Ebeid Makram

Eberbach $\mathrm{H}$

Ebner Nicole

Ebraheim $\mathrm{Na}$

Ebrahimi-Madiseh Azadeh

Eccles Ronald
Eccleston Christopher

Echeverria Sandra

Echt Katharina

Economopoulou P

Eden Karen

Edge Rhiannon

Edgren Suzan

Edlin Richard

Edman Kristina

Edmond Sara

Edmonds Michael

Edwards B

Edwards Duncan

Edwards John

Edwards Richard

Edwards Samuel

Efevbera Yvette

Efird Jimmy

Efstathiou Nikolaos

Eftimiou Orestis

Egan Matthew

Egeland Gm

Egger Garry

Eggins Liz

Eguchi H

Ehlers Anne

Ehrenthal Johannes

Ehret Dy

Ehrlich Joshua

Ehrmann Dominic

Ehteshami-Afshar Solmaz

Eibich Peter

Eifan Ao

Eikelboom Robert H

Eikey Ev

Einvik Gunnar

Eisenberg Yochai

Ekart R

Ekberg Stuart

Ekdahl Anne

Eklund Carina

Ekman Anna

Ekman Inger

Ekpenyong Chris

Ekuni Daisuke

El Mabchour Asma

El Morr Christo

El Tahlawi Mohammad

El Tantawi Maha

El-Baz Ayman

El-Mazny A

Eland Nicolaas

Elbaih Adel Hamed

Elbarouni Basem

Elbaz-Greener Gabby

Eleni Koroboki

Elf Jessica L

Elf Marie
Elfering Achim

Elfström Klara

Elgendy Islam

Elhassan Mohammed

Elia Nadia

Eliason Michele

Eliasson Mats

Elima Jedy-Agba Elima Jedy-Agba

Elinder Carl-Gustaf

Elizabeth Alcalde-Rabanal Jacqueline

Elkin Peter

Ellenberg Susan S

Elliman David

Elliott Rohan

Ellis Beverley

Ellis Catherine

Ellis Louise A

Ellis M

Ellsberg Mary

Ellwood David

Elmestekawy E

Elmore Joann

Elmusharaf Khalifa

Eloranta Sandra

Elovainio Marko

Elsebaie Hazem

Elsenburg Leonie

Elsharydah A

Elsinga Jelte

Elstad Jon

Elstad Jon Ivar

Elsworth Gerald

Elvander Charlotte

Elwan Mh

Elzein Fatehi

Emamifar Amir

Embleton Nicholas

Emilsson Louise

Emmert Maximilian Y

Emmerton Lynne

Emond Alan

Empana Jean-Philippe

Enblad Per

Endo Kenji

Endo Takaho

Ene-Iordache Bogdan

Eneqvist Ted

Engel Roger

Enguix-Armada Alfredo

Enki Doyo

Enoch Abigail

Enoch Mary-Anne

Enright Gemma

Ensor Joie

Enthoven Paul

Epari Venkatarao 
Ephraim Richard

Epple H -J

Epstein David

Eramudugolla Ranmalee

Erazo Marcia

Erdmann Matt

Erdoğan $\mathrm{H}$

Erez O

Ergina Patrick

Erichsen Andersson

Annette

Erichsen Rune

Eriksson Anders

Erkalp Kerem

Ermagun Alireza

Erne Paul

Ernst Edzard

Errett Nicole

Ersbøll Annette Kjær

Erskine Jonathan

Ervasti Jenni

Escarela Gabriel

Escher Monica

Eschler Anica

Esculier Jean-Francois

Esen Ui

Eskelinen A

Esplugues Ana

Estarlich M

Estcourt Claudia

Esterman Adrian

Eswaran Shanti Lynne

Eternod Carlos

Etminan Mahyar

Ettinger Lucas

Eurich Dean

Eusebi Leonardo

Eusebi Paolo

Euteneuer Frank

Evangelou Evangelos

Evans Charlotte

Evans David

Evans David

Evans Elizabeth

Evans Josie

Evans Nicola

Evered Lisbeth

Everett Tobias

Evers Andrea

Ewald Ben

Ewer Andrew

Eyles Jillian

Eyre Emma

Ezeh Osita

Ezpeleta L

Faasse K

Fabbo Andrea

Fabbri Giovanna
Faber Timor

Fabris Linda

Facchin Federica

Facchinetti Fabio

Facchini Sergio

Faconti Luca

Factor-Litvak Pam

Fadare Joseph

Fadden Grainne

Faedda Gianni

Faerstein Eduardo

Fahimi Jahan

Failde Immaculada

Fairbairn Nadia

Fajfr Miroslav

Fakhri Ali

Fakih Mohamad G

Falck-Ytter Corinna

Falcó Vicenç

Falconi Gabriele

Falk Delgado Alberto

Falk Hanna

Falk Stephen

Fallahzadeh Mohammad Kazem

Fallon V

Falsetti Lorenzo

Falster Michael

Falzon Dennis

Familoni Oluranti

Fan Amy

Fanfulla Francesco

Fang Chi-Tai

Fang Pengqian

Fang Yu

Fange Agneta

Fanning Jason

Fanshawe Thomas

Farag E

Faraoni D

Farias Mariela Granero

Faried Ahmad

Farina Nicolas

Farley Thomas

Farmaki Aliki-Eleni

Farmar Anna-Lisa

Farnaza A

Farndon Lisa

Farooqui Habib

Farquharson Barbara

Farquharson Kelly

Farr Michelle

Farre Albert

Farre Nuria

Farrell Lisa

Farrell Michael

Farrer Lou

Farrington Rebecca
Farsalinos Konstantinos E

Fary Robyn

Fasano Alfonso

Fasoro A

Fateh Abolfazl

Fatehi Farhad

Fauconnier Arnaud

Fauser Sascha

Faux S G

Favaloro Emmanuel J

Favero Giovanni

Favrat Bernard

Fawsitt Christopher

Fayanju Om

Fayfman Maya

Fazaa Alia

Fazel Seena

Fazeli Mir

Fear Nicola

Fearns N

Feaster Daniel

Fecht D

Fedele Francesco

Federolf Peter

Fedewa Alicia

Fedewa Stacey

Fedorowski Artur

Feeley Thomas

Fehlings Michael

Feig Denice

Feigenbaum Anna

Feinkohl Insa

Feizi Awat

Feldman Inna

Feldman Steven

Feldtkeller Ernst

Fellmeth Gracia

Fellows Jeffrey

Feng C

Feng Yibin

Feng Zhanchun

Fenger Runa

Fenoy Albert J

Fenton Miriam

Fenwick J

Fereshtehnejad

Seyed-Mohammad

Feresu Shingairai

Ferguson Cj

Ferguson Melanie

Ferket Bert

Fernanda Sousa Maria

Fernandes Lia

Fernandes Ra

Fernández Alba Jj

Fernández De Las Peñas

Cesar
Fernández-De-Las-Peñas César

Fernandez-Dominguez Juan Carlos

Fernández-Fernández Arroyo Matilde

Fernandez-Hidalgo N

Fernandez-Llatas Carlos

Fernandez-Luque Luis

Fernandez-Mendoza Julio

Fernandez Esteve

Fernandez Jordi

Fernandez Renae

Ferraccioli Gianfranco

Ferrajolo Carmen

Ferrari Giovanni

Ferrari Giulia

Ferrari Pietro

Ferrario Marco

Ferreccio Catterina

Ferree $\mathrm{S}$

Ferreira Daniela

Ferreira Giovanni

Ferreira Jason

Ferreira Joao Pedro

Ferrero Simone

Ferri Paolo

Ferris Jason

Ferro Mark

Ferroni P

Fewtrell Mary

Fichtenbaum Carl

Fidler Miranda

Fielding Richard

Fietze I

Figueiredo Ricardo

Figueras $\mathrm{F}$

Figueroa Jose

Filbay Stephanie

Filiopoulos Vassilis

Filippi Andrea R

Filippidis Filippos

Filippone Antonino

Filler Guido

Filocamo Giovanni

Filon Francesca Larese

Finch Naomi

Finch S

Finch Tracy

Finderup Jeanette

Finer Nicholas

Fineschi Vittorio

Finger Jonas D

Fink-Jensen Anders

Fink Astrid

Finkelman Robert

Finkelstein Eric

Finlayson Kathleen 
Finnane Anna

Finney Rutten Lila

Fiore Julio

Firn Mike

Fischbein Rebecca

Fischer Florian

Fischer Gayle

Fish Jennifer

Fishe Jennifer

Fisher Becks

Fisher Benjamin

Fisher Bernadette

Fisher Christopher

Fisher David

Fisher Eb

Fisher James M

Fisher Jane

Fisher Susan

Fitch Kenneth

Fitzgerald Anthony

Fitzgerald Karen

Fitzgerald Mark

Fitzmaurice C

Fjorback L

Flaatten Hans

Flachenecker Peter

Flandre Philippe

Flanigan Timothy

Flaxman Amy

Flay Brian

Flechtner-Mors M

Fleet Richard

Fleischer Nancy

Fleming Gini

Fletcher Carol

Fletcher Emily

Fletcher Ian

Fletcher Nick

Fletcher Sophie

Fleury Marie-Josée

Flight Ingrid

Florez Ivan D

Flower Andrew

Flowers Paul

Fluri Felix

Flynn Linda

Flynn Sarah

Fogarty Sarah

Foix-L'hélias Laurence

Fokom-Domgue Joël

Foley K

Foley M

Folgori Laura

Follestad Turid

Follmann Dean

Fone David

Fong Ss

Fontenelle Leonardo F
Fontes Luis

Foracchia Marco

Forbes Miriam

Ford John

Foresta C

Forma Leena

Formoso G

Fornai Francesco

Fornaro M

Foroutan-Rad Masoud

Forrest Simon

Forsberg Birger C

Forslund As

Forster Anne

Forsythe Laura

Fort Emmanuel

Fortin Marie-Chantal

Fortune Triona

Foskett-Tharby Rachel

Foster Claire

Foster Faye

Foster John

Foster Kr

Fotis Theo

Fournier Stephane

Fowles Jonathon

Fox Ck

Fox Greg

Frail Caitlin

Franceschi Silvia

Franceschini Gianluca

Francis David

Francis Joel

Francis Nick A

Franco-Paredes Carlos

Franco Alessia

Franco Israel

Francois Patrice

Franconi Giovanna

Frank Derk

Frank Erica

Franke A G

Fransen Mirjam

Fraser Gary

Fraser Gilles L

Fraser J

Frasnelli Johannes

Frat Jean-Pierre

Frazer Kathleen

Fredriksson Mio

Freedman Neal

Freedman Saul

Freeman Adrian

Freeman Karoline

Frega Antonio

Freitag Lutz

Freitas Hercules

Freitas Pf
Fremes Stephen

French Catherine

French Simon

Freni Sterrantino Anna

Frenkel M

Freund Yonathon

Frew Emma

Fridman Vera

Fridner Ann

Fried Eiko

Frighi Valeria

Frith Amy

Fritz Julie

Frizziero Antonio

Froessler Bernd

Frohlich Holger

Frola Claudia

Froom Paul

Frost Julia

Frost Lars

Frost Rachael

Frugé Ad

Frühbeck Gema

Fry Ian

Fu Dongbo

$\mathrm{Fu}$ Wah Ho Andrew

Fu King-Wa

$\mathrm{Fu} \mathrm{Vl}$

Fuchs Dietmar

Fuchs J

Fudge Nina

Fuentes Blanca

Fugh-Berman Adriane

Fujimoto Tsuguto

Fukuda Yoshiharu

Fukui Sadaaki

Fukushima R

Fulcher Jordan

Fullarton Gm

Fuller Brian M

Fuller Sebastian

Fung Teresa T

Funnell Martha

Furler John

Furlong Karen

Furnes $\mathrm{O}$

Furness Trentham

Furnham Adrian

Furukawa Shinya

Fusaro Maria

Fusco Francesco M

Füvesi J

Fyffe Ian

Gabbay Mark

Gaboury Isabelle

Gabutti Giovanni

Gacci Mauro

Gaccioli Francesca
Gadsby R

Gaffo Angelo

Gagliardi Anna

Gagnon Bruno

Gajewski Patrick D

Gal J

Galactionova Katya

Galan Alessandro

Galante Joseph

Galappatthy Priyadarshani

Galbraith Nd

Galceran Joan

Gale Christopher

Galfalvy Hanga

Galinasukoian GV

Gall John A M

Gall Seana

Gallacher Katie I

Gallagher Bernard

Gallagher Mw

Gallego Pedro

Galletti Ferruccio

Galloway James B

Galloway Jessica

Galvin Joseph

Galvin Rose

Galway K

Gammon D

Gamondi Claudia

Gan Earn

Ganann Rebecca

Ganczak Maria

Gander Jc

Gandra Sumanth

Ganesan Sudhir

Ganesh Aravind

Ganguli Anirban

Gant V

Gantz M

Ganu Daniel

Ganz Freda Dekeyser

Gao Wei

Gao X

Garbe Claus

Garchitorena Andres

Garcia-Aymerich Judith

Garcia-Calleja J

García-García José-María

Garcia-Garcia Lourdes

Garcia-Marcos L

Garcia-Perez A L

García-Sanjuán S

Garcia-Sempere Anibal

Garcia Beatriz

Garcia Olga

Garcia Paul

Garcia Rebecca

Garcia Rs 
Garden A L

Gardiner Fergus

Gardiner Joseph

Gardner Renee

Garelli Valentina

Garelnabi Mahdi

Garfield Sara

Garfield Sarah

Garg Priyanka

Garg Rajesh

Garg Rakesh

Garg Rk

Garg Satish

Garmo Hans

Garnas Kirsti

Garnett Sarah

Garnett Sarah P

Garrett Nigel

Garrido Juan

Garrido Melissa

Garstang Joanna

Gärtner Fania

Gary Jodie C

Garza Diego

Garza Mary

Gasche Christoph

Gascon Mireia

Gaskins Audre

Gasparyan Armen Yuri

Gassaway Julie

Gassner Jutta

Gatica-Rojas Valeska

Gau Ym

Gaucher Nathalie

Gaugler Joseph

Gauss Tobias

Gavaruzzi T

Gavazzi Cecilia

Gavin Anna

Gaweda Lukasz

Gazard Billy

Gazzard Gus

Ge Zhi-Zheng

Gea-Sánchez Montserrat

Gebauer Florian

Gebeyehu Endalew

Gebreab Samson

Gebremariam T

Gebremedhin Samson

Gebski Val

Geense Wytske

Gehring Ulrike

Geidne Susanna

Gelfond J

Gelli Aulo

Gelly Julien

Gelmetti C

Genberg Becky
Généreux Philippe

Generous Nicholas

Geng Zhi

Genovesi Simonetta

Gentles Stephen

George Ajesh

George Jaya A

George Johnson

George Steven

Georghiou Theo

Georgiadis Panagiotis

Georgiou Andrew

Georgiou Gabrielle

Georgousopoulou Ekavi

Gerada Clare

Geraghty Adam

Geraghty Keith

Gerardin J

Gérardin Patrick

Germeni Evi

Gerovasili Vasiliki

Gershlick Anthony

Gervas Camacho Juan

Gesesew Hailay

Geurts Dorien

Geyer Siegfried

Ghaderi Ata

Ghai Babita

Ghanbari Vahid

Ghanem Asaad A

Ghasem-Zadeh Ali

Ghayour Mobarhan Majid

Gholap Nitin

Ghose Bishwajit

Ghosh Dipansu

Ghoshal Uday

Giacco Domenico

Gialdini Gino

Giancipoli Ermete

Gianfagna Francesco

Giangregorio Lora

Giannicola Giuseppe

Giannoni Eric

Giannoni Margherita

Giannou Christos

Giannuzzi Viviana

Gianola Silvia

Gibbons Elizabeth

Gibbons Robert

Gibbs Jo

Gibertoni Dino

Gibreel Ahmed

Gibson-Smith Deborah

Gibson Cheryl

Gibson Grant

Gibson Josephine

Gichuru Phillip

Gidding Samuel
Gierlotka Marek

Gies Sabine

Gigli Gianluigi

Gijselaers Wh

Gil Efrat

Gilbert-Diamond Diane

Gilbert Clare

Gilbert Gl

Gilbert Ruth

Giles-Vernick Tamara

Giles Sally

Gill Hannah

Gill Stephen

Gill Tiffany

Gill Tom

Gillespie-Lynch K

Gillespie Brigid

Gillespie Catherine

Gillespie David

Gillespie Ulrika

Gilliam Melissa

Gillies Mike

Gilligan Conor

Gillis D

Gilson Richard

Gingerich Stefan B

Ginsberg Ann

Ginsberg Gary

Ginsberg Gm

Ginsberg Norman A

Ginsburg Lr

Giombini Lucia

Giorda Carlo

Giordano Raffaele

Giorgi Rossi Paolo

Giovannetti Elisa

Giraldo-Cadavid Luis

Girard Raphaele

Girardis Massimo

Girirajan Santhosh

Girling Alan

Girsen Anna

Gissane Conor

Gist Ramon

Gittoes N

Giuliani Alessandro

Giuliano Marina

Giustina Andrea

Gjeilo Kari Hanne

Gjeraa Kirsten

Glading Aj

Gladstone Beryl Primrose

Glangeaud-Freudenthal

Nine

Glantz S

Glantz Stanton

Glasby Jon

Glasgow Garrett
Glavind Julie

Glenn Nicole

Glenngard Anna

Glick David

Gliedt Ja

Glover Marewa

Glover Vivette

Gluud Lise

Gniadecki R

Goddard A

Godfrey Emma

Godin Judith

Godman Brian

Godoy-Monzon Daniel

Godtman Ra

Goedendorp M

Goel Sonu

Goessler Karla

Goettel Nicolai

Goetz Katja

Goff Sarah

Goffin John

Gogate Parikshit

Goh Jps

Goh Khean Jin

Goh Khean Lee

Goje Oluwatosin

Gokcen Neslihan

Göksu Erkan

Golberstein Ezra

Gold Christian

Gold Jason

Gold Lisa

Goldberg Andrew

Goldberg David

Goldberg Marcel

Goldblatt Peter

Goldenberg R

Goldman-Mellor Sidra

Goldsamt Lloyd A

Goldstein Edward

Golla Heidrun

Golladay Gregory

Golomb Beatrice

Goman Adele

Gomersall Judith

Gomes A

Gomes BC

Gomes James

Gomes Lilian

Gomes Nuno

Gomez De Terreros Javier

Gomez Real Francisco

Gomez-Roig M

Gomez Borja

Gomez Luis

Gonalez-Barcala Francisco

Goncalves Daniela A G 
Gonda Tamas

Gong Cathy

Gong Jianfeng

Gong Wenjie

Gonggalanzi Gonggalanzi

Gonya Jenn

Gonzales G

Gonzalez F Fernando

González-Arenas A

Gonzalez-Avila Georgina

González-Gay M

Gonzalez Alvaro

Gonzalez Esteban

Gonzalez Marianne

Gonzalez R Gilberto

Gonzalez Stevan

Good Gretchen

Goodacre Steve

Goodman David

Goodman Michael

Goodney Philip

Goodreau Steven M

Goodridge Donna

Goodwill Alicia

Goodwin Elizabeth

Goonesekera Sunali

Goorhuis A

Goossens Lucas

Goost Hans

Goovaerts Pierre

Goranitis Ilias

Gorczynski Paul

Gordon Adam

Gordon Aliza

Gordon Anthony

Gordon Barbara

Gordon L

Gordon Nancy

Gordon Sarah

Gorecka M

Goreczny Anthony

Gorini Giuseppe

Gorman Peter

Gorman Richard

Gorst Sarah

Gosens Taco

Göstemeyer Gerd

Götberg Matthias

Gothe N

Gotlib Conn Lesley

Goto Michihiko

Goto Rie

Goto Yoshikazu

Gotto Antonio M

Gotzmann Michael

Goulart Andre

Gould Jacqueline

Gould Lance
Goulden Robert

Goulding L

Gounden Verena

Govender Indiran

Goyal Nitin

Goyal V

Graber Cj

Graber Mark

Gracie David J

Gracner Tadeja

Graf William

Graham Cw

Graham Cynthia

Graham Hamish

Graham Simon

Grahame Nicholas J

Grainge Matthew

Grainger Rebecca

Gralla J

Grandahl Maria

Grandjean Peter

Granero-Molina J

Granger Cl

Grant Andrew

Grant William

Granziera Serena

Grasic Katja

Grasso Michael

Gratwohl Alois

Grau Maria

Grau Stefan

Graungaard A

Graven Peter

Gray Cindy

Gray Diane

Gray Janine

Gray Keith

Gray Laura

Gray Laura

Gray Peter B

Gray Ron

Grayling Michael

Grayling Mj

Grech Ever

Green James

Green M J

Green Mark

Green Peter

Greene-Cramer Blanche

Greenfield Geva

Greenfield Sheila

Greenhalgh Joanne

Greenhalgh Trisha

Greenslade Jaimi

Greenwald Hp

Greenway Steven

Greenwood Darren

Greenwood Nan
Greer Tracy

Gregory Ralph

Grenda Ryszard

Grenier Amanda

Grenon S

Gresham Ellie

Gresham Frank

Greve Anders M

Grieger Jessica

Griep Yannick

Griffin Xavier

Griffiths Ewen

Griffiths Gareth

Griffiths Peter

Grigg Celia

Grigorakos Leonidas

Grigoriev Pavel

Grigsby Timothy J

Grinshteyn Erin

Grissinger M

Grob Rachel

Groenewegen Peter

Grolez Guillaume

Grøndahl Vigdis

Abrahamsen

Grønning Kjersti

Grønseth Rune

Groom Km

Groome Michelle J

Groop Leif

Groseclose Sl

Grosman Benyamin

Gross Cynthia R

Gross Mm

Grossardt Brandon

Grosse Frie K

Grossman Brenda

Grossman Ellie

Grossman Shamai

Grossman Z

Grosso Giuseppe

Grubenhoff Ja

Gruber Maayan

Grübler Martin

Gruebner Oliver

Gruer Laurence

Gruessner Rainer

Grullich Carsten

Grunau Brian

Grund Birgit

Grundy Anne

Grundy Kate

Grünebaum A

Grzybowski A

Gualano Maria

Guan Jian

Guanira Juan

Guariguata Lenore
Guarnizo-Herreño Carol

Guasch Eduard

Guay Stephane

Gubio Aishatu

Gudiño Gomezjurado A

Gudziol Volker

Guerra Federico

Guerrero-López Cm

Guerriero Carla

Guerrini Renzo

Guest Jf

Gueyffier François

Guglielmo B Joseph

Guidotti Tee

Guilherme Flávio

Guirardello Edinêis

Guirgis Faheem

Guirguis L

Gulewitsch Marco

Guliani Harminder

Gulis Gabriel

Gulliford Martin

Gülmezoglu A

Gumber Anil

Gumede-Moyo Sehlulekile

Gunasekera Hasantha

Gundlund A

Gündoğar H

Gunes Harun

Gunnarsson Ulf

Gunther Carolyn

Gunzler Douglas

Guo Aimin

Guo Shigong

Guo X

Gupta-Wright Ankur

Gupta Navdeep

Gupta Rajeev

Gupta Resmi

Gupta Richa

Gupta Richa

Gupta Sumit

Gurevich Konstantin

Guru Khurshid

Gurudatt C

Gurusamy Kurinchi

Gustafsson Lars

Gustavson Kristin Brun

Gustavsson Catharina

Gutaj Pawel

Güthlin Corina

Guthrie Brian

Gutierrez Jose

Guttmacher Sally

Guure Chris

Guwatudde D

Guy Richard

Guyatt Gordon 
Guydish J

Guyton Jr

Gwini Stella

Gwynne-Jones David

Gwyther Holly

Ha In-Hyuk

Haahr T

Haas Marion

Haase M

Haby Michelle

Hackett Daniel

Hackshaw Allan

Haddad Fares

Haddock Christopher

Haddou Rahou B

Hadi Muhammad

Hadjichristodoulou

Christos $\mathrm{S}$

Haerkens Marck

Haeuser W

Hagan Cindy C

Hagedorn Hildi

Hagen Michael D

Hagg Sara

Hägglund G

Häggström Christel

Hagihara Akito

Hagiwara Shuichi

Haguenoer Ken

Hahlweg Pola

Hahn P

Hahne Andrew

Haile Melaku

Haile S

Hailey David

Haines-Saah Rebecca

Hajarizadeh Behzad

Hajjar Emily

Hajjou M

Hak Eelko

Haldre K

Haley Valerie

Hall Abigail

Hall Alix

Hall Charles

Hall KK

Hall Martin

Hall Peter

Hall R

Hall Ruth

Hall Work

Hallbook Helene

Halldner Linda

Halle Ekane Gregory Edie

Haller Guy

Haller Mj

Hallgren Kevin

Halliday Alison
Halligan Sarah

Halonen Jaana

Halpern P

Halstead Scott

Halvorsen Kjell

Hamad Rita

Hamade Prudence

Hamadneh Shereen

Hamasuna Ryoichi

Hamer Mark

Hamidi Samer

Hamilton-Craig Christian

Hamilton Clayon

Hamilton David

Hamilton Jean

Hamilton Leah

Hamilton Willie

Hammerle Florian

Hammerton Gemma

Hammett Theodore M

Hammill Chet

Hammond William

Hampshire Kate

Hamrefors Viktor

Han Jin H

Han Kihye

Han Liyuan

Han Thang

Handley Tonelle

Hanley Ja

Hanley Janet

Hanlon Alexandra

Hanlon Peter

Hanmer J

Hanna Lisa

Hanney Stephen

Hannigan A

Hanrahan Lawrence

Hansen Bo T

Hansen Cs

Hansen Kim

Hansen Marco

Hansen Marianne

Hanson Erik

Hansoti Bhakti

Hansson Oskar

Hansson Per Olof

Hanzal Engelbert

Hao Wenrui

Hao Yuantao

Harada Kaito

Harcombe $\mathrm{H}$

Harcombe Zoe

Hardcastle Timothy

Harder Thomas

Harding Katherine E

Harding Keith

Hardt Jochen
Hardy Louise L

Hareendran Asha

Harenberg Job

Hargadon Dp

Hargreaves Sally

Harju Jarkko

Harle Christopher

Harling Guy

Harmancey Romain

Harnoss J

Haroutiunian Simon

Harrigan Mark R

Harris Cory

Harris H R

Harris Ian A

Harris John

Harris Joshua

Harris Mark

Harris Sophie

Harris Taylor

Harrison Dening Karen

Harrison G J

Harrison J

Harrison Jim

Harrison Reema

Harrison Samantha

Hart Laura

Hart Nicolas

Hart Roger

Hartl Roger

Hartman Nicholas

Hartmann Anders

Hartmann Miriam

Hartrick Craig

Hartsell Zachary

Hartung Daniel M

Hartung Hans-Peter

Hartung Olivier

Hartung $\mathrm{Tj}$

Hartzler Bryan

Harvey Merryl

Harville Emily

Harwood L

Harwood Rowan

Hasan J

Hase Ryota

Hashimoto Hideki

Hashimoto Simone

Hasman A

Hassan-Smith Z

Hassan Abdullahi

Hassan Louise

Hassany Mohamed

Hasselberg Marie

Hassiotis Angela

Haste A

Hastert Ta

Hatam Nahid
Hatch Stephani

Hatchard Jenny

Hatcher Abigail

Hatfield Dan

Hatzopoulos S

Hauck Yvonne

Haugen A

Haukenes Inger

Hausenblas Heather

Hausfater Pierre

Havard Alys

Havas Kathryn

Havenetidis Konstantinos

Havyer Rachel

Haw $S$

Hawk C

Hawkes M

Hawkins Dm

Hawley Carol

Hawley Pippa

Hawley Sarah

Hay Bill

Hay Jennifer L

Hayakawa Masahiro

Hayashi M

Hayden Jacky

Hayden John

Hayhoe Simon

Haymart Megan

Haynes Ab

Haynes R Brian

Hayney Mary

Hays Meredith

Hays R

Hayward Andrew

Haywood Philip

Hazell Philip

Hazra Avijit

Hazra Avishek

He Huan

He Kevin

He Nianan

He T

He Vincent Y F

He Yani

He Yutong

He Zhe

Head Michael

Healy David

Hean Sarah

Heaney Jennifer

Heard I

Hearld Lr

Heaslip Vanessa

Heckemann Birgit

Heckman Carolyn

Heckman George

Heczko Pb 
Hedberg Jakob

Heerman Wj

Heesch Kristiann

Heesen Christoph

Hefermehl Lukas

Hegade Vinod

Heggie Robert

Heijink Irene

Heinrich Joachim

Heinrich Joachim

Heinze Georg

Heit John

Heitzinger Kristen

Hejtmancik J Fielding

Helbok Raimund

Heley Stella

Hellerstein S

Hellgren Margareta

Helliwell Philip

Hellström Ingrid

Hellström Sten

Helmerhorst Frans

Helmy Remon

Helseth Ragnhild

Hemilä Harri

Hemming Karla

Henchoz Yves

Henderson Claire

Henderson Jane

Henderson Joanna

Henderson Julie

Henderson Max

Henderson Paul

Hendrick Paul

Hendricks Peter

Hendriks Sheryl

Hendry Maggie

Henneberg Maciej

Hennemann Severin

Hennessy Deirdre

Henningsen A

Henrard S

Henriksen Anne H

Henriksen Marius

Henry Cj

Henshaw Helen

Hentschke Marta

Hentzien Maxime

Herbert Amanda

Herbison Peter

Herbolsheimer Florian

Herdeiro Maria

Herder Matthew

Herdman Michael

Herly Mette

Hermanides J

Hermanns Norbert

Hermansson Ann
Hernan Andrea

Hernández Morante Jj

Hernandez-Andrade E

Hernandez-Padilla Jose

Hernandez Inmaculada

Herrington William

Herrmann-Lingen

Christoph

Hersch Jolyn

Herzig Lilli

Herzog Thaddeus

Hess Lm

Hetherington Erin

Heuch Ivar

Heukelbach Jorg

Hewett Nigel

Hewitt Ian

Hex Nick

Hey Spencer

Heydtmann M

Hibbert Peter

Hicken Margaret T

Hickey Martha

Hicks Blainaid

Hicks Matthew

Hicks Rw

Higashi Yukihito

Higgins Gerald

Higgins Stephen

Hignett Sue

Higuchi Yoshinori

Hikichi Hiroyuki

Hikichi Hiroyuki

Hilden Jørgen

Hildesheim Allan

Hiles S A

Hilfiker R

Hilfiker Roger

Hill Andrew

Hill Anne-Marie

Hill Briony

Hill Jenny

Hill Jo

Hill Sarah

Hill Terrence

Hill Z

Hillen J

Hillier-Brown Fc

Hillier Susan

Hillis Argye E

Hillman Magnus

Hincapie Ana

Hinckson Erica

Hinde Andrew

Hinde Sebastian

Hinic Darko

Hinson Holly

Hipwell Alison
Hirakawa Yoshihisa

Hirani Rena

Hirata $\mathrm{H}$

Hiremath Swapnil

Hirko Kelly

Hirokawa Kumi

Hirota Kazuyoshi

Hirotsu Camila

Hirschfeld S

Hirsh Yechezkel Galit

Hirshhorn Andrew

Hirst Yasemin

Hiscock Harriet

Hiscock Harriet

Hiscock Rosemary

Hittel Dustin

Hjollund Niels

Ho-Pham Lt

Ho Mandy

Ho Roger C M

Ho Vivian

Hoag Jessica

Hoaglin David

Hoagwood Kimberly

Hoare Derek

Hobbs Knutson K

Hobin Erin

Hobson Esther

Hockenberry J

Hockly Megan

Hodge Allison

Hodgson Carol

Hodgson Rob

Hodson Karen

Hoehn Richard

Hoek Janet

Hoekstra Trynke

Hoenigl Martin

Hoerger Thomas

Hoffman Daniel

Hoffman Suma

Hoffmann-Vold

Anna-Maria

Hoffmann $\mathrm{Cj}$

Hoffmann Kathryn

Hofhuis Jose G M

Hofmann Bjoern

Hofmann-Wellenhof

Rainer

Hofmann Bjørn

Hofmeyr G Justus

Hogan Helen

Högenauer C

Hogenbirk John

Holanda Vanessa

Holder Holly

Hole Grete

Holford Theodore
Holick Michael

Hollestein Lm

Holliday Richard

Hollinger Alexa

Hollingworth Samantha A

Holly Cheryl

Holly Jeff

Holm Ellen

Holm Lena

Holm Manne

Holman Daniel

Holmberg Christine

Holmberg Lars

Holmer H

Holmes-Rovner Margaret

Holstein K

Holt Elizabeth

Holt P

Holt Richard Ig

Holt Tim

Holzinger Felix

Hom Lisa A

Homayounfar R

Homer Caroline

Homlong Lisbeth

Homma Sakae

Honda Sumihisa

Honda Yasushi

Honeybul Stephen

Honeyford Kate

Hong Ya

Hong Hwanhee

Hong Sang-Bum

Hong Xuezhi

Hong Yoonki

Hooker Angelo

Hooker Rod

Hooper Gary

Hooper Richard

Hoos David

Hope Aluko

Hopkins Richard S

Horgan Anne

Horgan Frances

Horgan S

Horing Bjoern

Horio Masaru

Horne Maria

Hornung Rick

Horowitz John

Horton Susan

Horvat Nejc

Hosie Annmarie

Hosking Joanne

Hoskins Iffath

Hoskins R

Hossain Ahmed

Hossain Shahed 
Hossain Zakia

Hossein Z

Hosseini Abolfazl

Hosseini Mg

Hotait Hassan

Hotchkiss Rs

Hou Gui-Cheng

Hou Zhiyuan

Hough J

Houghton Serena

Houpt Eric

Houssami Nehmat

Houssiau Frederic A

Houwen Juul

Houwing Rh

Hovén Emma

Hovlid Einar

How Jacques

Howard Lauren

Howard Michelle

Howe Edmund

Howe Ts

Howell Debra

Howell M

Howell Martin

Howell Simon

Hoxha Ilir

Hoyer Erik H

Hoyos Juan

Hozumi Hironao

Hsiang John C

Hsiao Chiu-Yueh

Hsieh Cheng

Hsieh Shi-Jun

Hsieh Yu-Hsiang

Hsu Amy

Hsu Jason C

Hsu Po-Kuei

Htwe Than Than

Hu Guoqing

$\mathrm{Hu}$ Hui

$\mathrm{Hu} \mathrm{J}$

Hu Jun

Hu Ruwei

$\mathrm{Hu}$ Susan

Hu Xiao-Min

Hu Yan

Huak Chan

Huang Cc

Huang Chin-Chou

Huang Eileen

Huang Jennifer

Huang Jing

Huang Kai-Wen

Huang Lian-Hua

Huang Qiang-Min

Huang Ruixue

Huang Shi
Huang Shih-Han

Huang Weidong

Huang Yixiang

Huang Yong

Huang Yuli

Huang Zhigang

Huber Kurt

Hubloue I

Huddy Jeremy

Hudgel David W

Hudgins Joel

Hudon Catherine

Hudson Benjamin

Hudson Darrell

Huen Karen

Huerta Consuelo

Hueso-Montoro Cesar

Huffman Sandra

Hughes A

Hughes Carmel

Hughes Christopher

Hughes Nathan

Hughes Nicole

Hughes R

Hügle T

Hugon Jacques

Huguet Nathalie

Huhn Evelyn

Hui A

Hui David

Huirne Judith

Huisman Albert

Hulme Adam

Hulme Jennifer

Huls Anke

Hulzebos HJ

Humberg Alexander

Hume Patria

Humphrey John M

Humphreys Cathy

Humphreys Mitchell

Humphries Debbie

Humphries Misty D

Humphries Stacey

Hundley Vanora

Hunt Abby

Hunter Benton

Hunter Nezahat

Hunter Ruth

Hunter Sharyn

Huntington-Moskos Luz

Huntley Alyson

Hurley Deirdre

Hurley Emily

Hurley John

Hurley Margaret

Hurst Samia

Husain Nusrat
Husain Zakir

Hussain Mehwish

Husson $\mathrm{O}$

Hutchison Coll

Hutfless Susan

Hutti Mh

Huttner Benedikt D

Huupponen Risto

Huurneman Bianca

Huybregts Lieven

Huynh Chi

Hwang David Y

Hwang Ula

Hyde Matthew

Hyldig Nana

Hyrich Kimme

Hysong Sylvia

Hyun Insoo

Hyun Kk

Gautham Meenaks

Iacobelli Silvia

Iagaru Andrei

Iannaccone Mario

Iavicoli Ivo

Ibrahim H N

Ibrahim Kinda

Ibrahim Toni

Icick $\mathrm{R}$

Idris Iskandar

Iemitsu Motoyuki

Iftikhar Imran $\mathrm{H}$

Iga Jun-Ichi

Igaz Peter

Igland Jannicke

Iglesias Verónica

Iguchi Seitaro

Ihara Masafumi

Iikura M

Ike Anthony

Iles Ross

Iliopoulos Efthymios

Illades-Aguiar Berenice

Imaiso Junko

Imms Christine

Imperatore Nicola

Inaba Hideo

Inaguma $\mathrm{S}$

Inai Kunihiro

Inauen Jennifer

Indrayan A

Ingemarsdotter Persson

Kristina

Inglehart $\mathrm{Mr}$

Ingram Jenny

Iniesta Raquel

Innes Anthea

Innes Stanley

Innis Jennifer
Innominato Pasquale

Inokuchi Mikito

Inoue Sumito

Inoue Taku

Inskip Hazel

Intepeler Ss

Inthout Joanna

Inui Thomas

Iqbal Zaheen Ahmed

Iribarren C

Irimia Andrei

Irizarry $\mathrm{T}$

Irving Kate

Irving Sharon Y

Irwin Adam

Irwin Rachel

Isa Modibbo Fatima

Isaac Vivian

Isaacs Anton

Iserbyt Peter

Isgor Z

Ishihara Toru

Ishikawa Kazuya

Ishikawa Takamichi

Islam Akm Monwarul

Islam Ashraful

Islam Farah

Isles Chris

Ismail Khaled

Issa Js

Isaac Amanda

Itabashi Ryo

Ittenbach Richard

Itzel Eva

Iudici Michele

Ivanov Volen

Ivers Rebecca

Iversen Hilde

Ivory Vivienne

Iwamoto Takayuki

Iwasaki Masanori

Iyengar Sriram

Iyer Hari S

Iyngkaran Pupalan

Izadi Hooshang

Izumiya $\mathrm{Y}$

Jørgensen Karsten

Jaakkimainen R Liisa

Jabbari B

Jacinto Tiago

Jackowska Marta

Jackson T

Jackson Alun

Jackson Alun

Jackson Cath

Jackson Louise

Jackson R

Jackson R 
Jackson Rod

Jacobi Judith

Jacobo-Estrada Tania

Jacobs Colin

Jacobs Phil

Jacobs Robin

Jadoo Saad Ahmed Ali

Jaenisch Thomas

Jaensson Maria

Jahanfar S

Jain Akanksha

Jaisoorya Ts

Jake-Schoffman Danielle

Jakovljevic Mihajlo

Jakovljevic Mihajlo B

Jakubovic Raphael

Jallad Karl

Jamal Shahin

James Michael L

James Peter B

James Stefan

Jamsen Esa

Janardhana N

Janda Monika

Jang Myoungock

Jang Sung-In

Jang Timothy

Jani Ilesh

Jannini Ea

Jansen Floor

Jansen Jesse

Jansen R

Janssen Patricia

Janssens Astrid

Janwantanakul Prawit

Jaquet A

Jardim Thiago

Jardri Renaud

Jarosz Chobot Przemysława

Jaruratanasirikul Somchit

Jarvinen Teppo

Jauhar Sameer

Javed Athar

Jawad Mohammed

Jayadevappa Ravishankar

Jayaraman Sudha

Jayasena Rajiv

Jayasuriya Vathsala

Jeffs Lianne

Jelinek H F

Jelle Visser Benjamin

Jelsma Dorothee

Jenkins Catharine

Jenkins Cathy

Jenkins Paul

Jenkins Rachel

Jenkins Renee

Jenny Firth-Cozens Jenny
Jensen BT

Jensen Erik

Jensen Hanne

Jenson Alexander

Jeon Sangchoon

Jeong Ki

Jerath Angela

Jeschke Marc

Jessani Nasreen

Jeter $\mathrm{Cb}$

Jetha Arif

Jewell Jo

Jeyaseelan L

Jeyaseelan Lakshmanan

Jha Vivekanand

Jia Yongliang

Jiang Jun

Jiang Depeng

Jiang Joyce

Jiang L

Jiang Lixin

Jiang Luohua

Jiang Qing

Jiang S H -T

Jiang Yannan

Jiang Yu

Jick Susan

Jimenez-Garcia Rodrigo

Jiménez-Mejías E

Jiménez José R

Jirón Marcela

Jiskra J

Joag Kaustubh

João Cordeiro Da Costa

Jochem Warren

Joeris Alexander

Johannessen Karl-Arne

Johannsson Gudmundur

Johansen Adam

Johansen Ingrid $\mathrm{H}$

Johansson Klara

Johansson L

Johansson Tim

John Denny

John Garry

Johns Benjamin

Johnson-Agbakwu Crista E

Johnson Claire

Johnson Fr

Johnson Judith

Johnson Karin G

Johnson Linda SB

Johnson Michelle

Johnson Michelle

Johnson Newell

Johnson Niall

Johnson William

Johnston Desmond
Johnston Grace

Johnston Samantha

Johnston Venerina

Jolly Kate

Jolly Sanjit

Jommi Claudio

Jones Andrew

Jones Andrew

Jones Ashlin

Jones Carys

Jones Christopher

Jones Damon E

Jones Daniel

Jones David

Jones K

Jones K

Jones Laura

Jones Mark

Jones Meinir

Jones Richard

Jones Rl

Jones Rodney

Jones Simon

Jong Gwo-Ping

Jongen Joost

Jongenelis Michelle

Jonkman Nini

Jönsson Eg

Jonsson Emma

Jordan Jo

Jordan S

Jordan Susan

Jorgensen Andrea

Jørgensen Christoffer

Jorgensen Lars

Jorgenson Eric

Jorm A

Jorm C

Jörres Achim

Jose Tarazona-

Santabalbina Francisco

Joseph Bobby

Joseph Rw

Joshi Anand

Joshi Heather

Joshi Nabin

Joshi Rohina

Joshy Grace

Jouvent E

Jovic Dragana

Jowsey Sheila

Jowsey Tanisha

Joy Mark

Joyce-Brady Martin

Juárez Sp

Juby A

Judd E

Judd Jenni
Juffermans Nicole

Juillard Catherine

Julious Steven

Jull Andrew

Jull Gwen

Jung-Choi Kyunghee

Juonala Markus

Juraschek Stephen

Jurisson M

Kabata P

Kabboord A

Kabel Allison

Kachur Sergey

Kaciroti Niko

Kadam Umesh

Kaddumukasa Mark

Kadir Muhammad

Kadom Nadja

Kadotani Hiroshi

Kaewkungwal Jaranit

Kagina Benjamin

Kağıtcı Mehmet

Kahan Scott

Kaimal Girija

Kaiser Christoph

Kaji Aiko

Kakehashi Akihiro

Kakeman Edris

Kalakoti Piyush

Kalb Luther

Kalbfleisch Jack

Kaleta Dorota

Kalhan Satish

Kalincik T

Kalkman Cor

Källander Karin

Kalmakis Ka

Kalokhe Ameeta

Kalra Sundeep

Kalva Sanjeeva P

Kamal Madeeha

Kamimura Hiroteru

Kaminsky La

Kamperidis Nikolaos

Kampmann Ulla

Kana Musa

Kanaan Mona

Kanagasundaram Suren

Kananura Rornald M

Kandala N

Kandati Jithendra

Kander Thomas

Kane Eleanor

Kane Nancy M

Kane R

Kane Sumit

Kanerva Anne

Kang $\mathrm{H}$ 
Kang Hyunsik

Kang Jing

Kang Jinyong

Kang Jy

Kang Melissa

Kang Mo-Yeol

Kanic V

Kankaanpaa Eila

Kanste Outi

Kapellas Kostas

Kaphle Dinesh

Kapila Vansh

Kaplan Bekir

Kaplan Lauren

Kaplan Robert

Kapur Kush

Kar Sumit

Kara Ibrahim

Karageorge Aspasia

Karagiannis Thomas

Karahalios Emily

Karakala Nithin

Karakulak U

Karamouzian Mohammad

Karande S

Karanja J

Kardefelt-Winther Daniel

Karim Md

Karimi-Sari Hamidreza

Karimkhani Chante

Kario Kazuomi

Karkouti K

Karlawish Jason

Karlsen Bjorg

Karlson Elizabeth

Karlsson Linn

Karlsson Linnea

Karras Alexandre

Karsies T

Karthaus Meinolf

Kashiwagi Mitsuru

Kashyap Rahul

Kasim Adetayo

Kassavou Katerina

Kassianos Angelos

Kastritis Efstathios

Kastrup M

Katanoda Kota

Katavić V

Kateeb Et

Katende David

Kates Stephen

Kates Stephen

Katheria Anup

Kato Bernet

Kato K

Kato K

Kato Masahiko
Kato Motohiro

Katsikatsou M

Katsoulis M

Kattelmann Kendra

Katz Alan

Katz Bp

Katz Mark

Katzenellenbogen Judith

Katzman Wb

Katzman Wendy

Kaufman-Shriqui Vered

Kaufman Dave

Kaufman Elinore

Kaufman N

Kauppila Timo

Kaur Prabhdeep

Kaushal N

Kaussner Yvonne

Kauvar David

Kavati Abhishek

Kavut A Baccioglu

Kawaguchi Yoshiharu

Kawamoto Ryuichi

Kay Margaret

Kaya Mutlu Ebru

Kaya Derya Ozer

Kayambu Geetha

Kayem Gilles

Kayode Gbenga

Kayyali Reem

Kazi Dhruv

Kazis Lewis

Keage Hannah

Kearne Phillip E

Kearney Patricia

Kearns Ben

Kearns Therese

Keaver Laura

Keay Lisa

Kebschull M

Keeble Claire

Keegan Thomas

Keel Stuart

Keelan Ja

Keeling David

Keep Jeff

Keers Richard

Kegler Michelle

Keith Rachel

Kelestimur Fahrettin

Kelle Bayram

Kelleher Ian

Keller Frieder

Keller Gisela

Keller Sara

Keller Ulrich

Kellermayer Richard

Kellett John
Kelley George

Kelley Megan

Kelling Sarah

Kelly Brian

Kelly Claire M

Kelly Helen

Kelly Maria

Kelly Michelle

Kelsall Helen

Kelsey Tom

Kemeny Andras A

Kemp Karen

Kemp Lynn

Kempert Heidi

Kenardy Justin

Kendal Sarah

Kendall Claire

Kendzerska T

Kengne Ap

Kengne Andre

Kenne Deric

Kennedy Deborah

Kennedy Mary-Claire

Kennedy Norelee

Kennedy Ryan David

Kenny Amanda

Kenny Ursula

Kent Erin

Keogh B

Kern W V

Kerr Jacqueline

Kerr Jelani C

Kerry Sally

Kesim Murat

Keskin Gulseren

Kessing Lars

Kessler Ian

Kesten Joanna

Kesztyüs Dorothea

Kettel Khan Laura

Ketteler Markus

Keville Saskia

Khabaz Mafinejad

Mahboobeh

Khadjesari Zarnie

Khalafallah Alhossain

Khalid S

Khalil Hanan

Khamisa Natasha

Khan Asad

Khan Ahad

Khan Alisa

Khan Erum

Khan $\mathrm{Hr}$

Khan Inam

Khan Jahangir

Khan M

Khan $\mathrm{Mj}$
Khan Moin

Khan Rabia

Khan Salah Uddin

Khan Sobia

Khan Tahir Mehmood

Khanal Santosh

Khanam Rasheda

Khanassov Vladimir

Khanna P

Khanolkar Amal R

Khashan Ali

Khateeb Elham

Khazaal Yasser

Khdour Maher R

Kheirallah Khalid

Khemani Robinder G

Khera Sahil

Khetan Aditya

Kho Michelle

Khoja Samannaaz

Khoo Joan

Khorgami Zhamak

Khubchandani Jagdish

Khurshid Shaan

Kiani Arda

Kiberd Bryce

Kiberu Vincent

Kidd L

Kielland Nb

Kietzmann Diana

Kildea Sue

Kilis-Pstrusinska Katarzyna

Kiljanski Jacek

Kilkenny Monique

Killick Sally

Kilminster Sue

Kilpatrick M

Kilpatrick Sarah

Kim Dae Jung

Kim Chanmin

Kim Dong-Hyun

Kim Hyengsu

Kim Hyoung-Ryoul

Kim Jihye

Kim Jinseok

Kim John

Kim Jong In

Kim Joosup

Kim Kyong-Jee

Kim Kyoung-Kon

Kim Kyung Mi

Kim L

Kim Mi-Na

Kim Nak-Kyeong

Kim Seok-Woo

Kim Susan

Kim Sw

Kim Tae 
Kim Woohyeon

Kim Yoon-Bum

Kim Youngmee

Kimbell Barbara

Kimberlee Richard

Kimmerle Joachim

Kind Paul

Kindblom Jenny

Kindzierski Warren

King Kevin S

King Brian

King C

King Mark

King Nicola

Kingsbury Sarah

Kini Annapoorna

Kinner Stuart

Kinouani Shérazade

Kinross James

Kinsman Leigh

Kiosses Dimitris

Kippler Maria

Kirby Andrew

Kirby George

Kirby Russell

Kirchhof Paulus

Kirkham Fj

Kirkman Allison

Kirkman Maggie

Kirschner Benny

Kirtschig G

Kishore Amit

Kissane David

Kitagawa Masashi

Kiviniemi Tuomas

Kivlehan Sean

Kjellgren Karin

Klaiman Tamar

Klasen $\mathrm{H}$

Kleber Franz

Kleiman Em

Klein Jens

Kleiner Ana Francisca

Klemanski Dori

Klemetti Reija

Klewer Jörg

Klimentidis Yann

Kline Gregory A

Klinga Charlotte

Klocker Helmut

Klodnick Vv

Klompas Michael

Klonizakis Markos

Klopotowska Joanna

Klotsche Jens

Klouche S

Klug Stefanie

Kluijtmans Manon
Kmail Zaher

Knaevelsrud Christine

Knaggs Roger

Knapp Guido

Knerr Sarah

Knibb Rebecca

Knibbs Luke

Knight Aimee

Knight Gwen

Knight Marian

Knittle Keegan

Knobe M

Knoll Maria

Knoop J

Knoop Jesper

Knowles Zoe

Knudson Duane

Knüppel Sven

Ko Young

Kobayashi Lindsay

Kobayashi Masatake

Kobelt Gisela

Köberlein Juliane

Kocherlakota P

Kochilas Lazaros K

Kodadek Lm

Kodani Eitaro

Kodjebacheva Gergana

Koffi Alain

Koffman Jonathan

Kofoed Kf

Kohlboeck Gabriele

Kohli Anjalee

Kohn Martin

Koivusilta Leena

Koizumi Akio

Koji Oba

Kojima Kazuyuki

Kok Sang-Heng

Kok Victor

Kokame GT

Köke Albere

Kokkinen Lauri

Kolben Theresa

Koldoff Ea

Kolfschoten N

Kolias Angelos

Kollath-Cattano C

Komasawa Nobuyasu

Komatsu Shunichiro

Kompoti Maria

Konde Sapna

Kondo Takahisa

Kones Richard

Kong Kai

Koning Maaike

Konkolÿ Thege Barna

Konnopka A
Konopasek Lyuba

Konradsen Hanne

Konstantinides Stavros

Konstantinos Votis

Kontopantelis Evangelos

Konturek Peter

Koo Winston

Kooienga Sarah

Kool Bridget

Koopman James

Koornneef Erik

Kopanitsa G

Kopinska Joanna

Aleksandra

Kopp Lisa M

Koren Gideon

Korenromp Eline L

Korenstein Deborah

Kornak J

Kornej Jelena

Kornhaber Rachel

Kortum Karsten

Korzeniewski Sj

Kose Samet

Koskenvuo Karoliina

Kosmidou Ioanna

Kosofsky Barry E

Koster Es

Koster Matthew

Kostev Karel

Kostovski E

Kostuj Tanja

Kosugi Tomoki

Kotagal Vikas

Kotronoulas Grigorios

Kottner Jan

Kotwal Atul

Kotz Daniel

Koukounari Artemis

Koul Parvaiz A

Kouladjian O'donnel Lisa

Kounis Nicholas

Kourelis TV

Koutros Stella

Kovach Christine R

Kovacs Burns Katharina

Kovacs Caroline

Kowada A

Koyama Shihoko

Kozaki Koichi

Kraehenbuhl Bern Lukas

Kraemer Hc

Kraemer John

Kraeutler Matthew

Kraft-Todd Gordon

Kragholm Kristian

Krahn Murray

Krall J
Kramer Daniel

Kramer Diether

Kramer Sharon

Krans Julie

Kratky J

Krause D

Krause Niklas

Krauss Inga

Kraut Allen

Kravitz Richard

Krebs Jeremy

Krebs Jörg

Krebs Nm

Kredo Tamara

Kreitschmann-Andermahr I

Kress John

Kreuter Alexander

Kreuter Michael

Krings Timo

Krishnadath Ingrid

Krishnan Dr Jerry A

Krishnan Shilpa

Krismer Bernhard

Krist Lilian

Kristensen Lars Erik

Kristensen Solvejg

Kristoffersen Doris

Kristoffersen Doris Tove

Kriston Levente

Kritsotakis Ei

Krive J

Krivitski Nikolai

Krivoy Amir

Kř́̌žová Eva

Krleža-Jerić Karmela

Kroemer Nils

Krogh J

Krokidis Miltiadis

Kroll David S

Kronborg Lise

Kronish Ian M

Kroon Feline

Krueger Joachim

Kruger Immie

Kuchar E

Kuchenbaecker Karoline

Kuchinsky Stefanie

Küçük Mert

Kucukler Nagehan

Kudo Hideo

Kueh Yc

Kufa Tendesayi

Kufe Nyuyki

Kuh Diana

Kuhn Christine

Kuhn Elizabeth

Kuhn Eric 
Kühn T

Kuijer P Paul

Kuk Jennifer

Kukimoto I

Kulatilake Anita

Kulczycki Andrzej

Kulik Alexander

Kullgren Jeffrey

Kulzer B

Kumagai Koshi

Kumar Atul

Kumar Gautam

Kumar N

Kumar Sheila

Kumar Va

Kump P

Kumsa Legese

Kunej T

Kunik Mark Edwin

Kunisak Sm

Kunst Anton

Kunst Gudrun

Kuntz B

Kunutsor Setor

Kunz Regina

Kuo Chang-Fu

Kuo Chin-Chi

Kupeli Serhan

Kuper Hannah

Kuperman Ef

Kupka M

Kuppuswamy Anna

Kuptniratsaikul Vilai

Kurdi Amanj

Kureshi F

Kuriyama Akira

Kurjak Asim

Kurjanowicz P

Kurrle Susan

Kurtul A

Kuru Nilgün

Kurz Alexander

Kusano As

Kusano Motoyasu

Kusurkar Rashmi

Kutryba Basia

Kuvin Jeffrey

Kuwabara Masanari

Kuys Suzanne

Kvarstein Gunnvald

Kvernmo Siv

Kwan J

Kwan M

Kwang Ng

Kwegyir-Afful Emma

Kweon Meera

Kwok Chun

Kwok Man Ki Maggie
Kwok Richard

Kwon Hyuksool

Kwon Ohyun

Kwon Seungwon

Kwon Younghoon

Kyaw Moe

Kyle Theodore

Kypridemos Chris

Kyratsis Yiannis

Kytö Ville

Kytö Ville

Löwe Bernd

La Caze Adam

La Rosa Valentina

La Torre Giuseppe

La Vecchia Carlo

La Vigne Nancy

Laban $\mathrm{Cj}_{\mathrm{j}}$

Laberge Luc

Labrador Barba Elena

Labrague Leodoro J

Lacey Krim

Lachat Carl

Lachman Peter

Lachowsky Nathan J

Ladd Elissa

Ladden Maryjoan

Lader Malcolm

Ladyzynski Piotr

Laflamme Lucie

Lagali Neil

Lagerlof Maria

Laging Bridget

Laguzzi Federica

Lahti Mari

Lai Chao-Lun

Lai Chih-Kuan

Lai Meng-Chuan

Lai Nai Ming

Lai Yu-Hung

Laidlaw Anita

Laimi Katri

Laird Eamon

Laisaar Kaja-Triin

Laitinen Kirsi

Lakerveld Jeroen

Lakhi Nisha

Lala Rizwana

Laliberte Maude

Lalla-Edward Samanta

Lally Philippa

Lam Eddie T C

Lam Lawrence

Lam Tai Pong

Lamadrid-Figueroa Hector

Lamb Benjamin

Lambalk Cornelius

Lambert-Kerzner Anne
Lambert Bruce

Lambert Veronica

Lambert W Clark

Lambooij Mattijs

Lameire Norbert

Lamont Tara

Lamontagne Francois

Lamoureux Ecosse

Lan Ching

Lanaspa Miguel

Lancee Jaap

Lanceley Anne

Lancia Loreto

Land Lucy

Landefeld C

Landercasper Jeffrey

Landoni Giovanni

Lane Christianne

Lane Steven

Lang Andras

Lang Chim C

Lang Eddy

Lang Sc

Lang Wan

Langan Sinead

Langendam Miranda

Langer Bernhard

Langewitz Wolf Axel

Langhammer Arnulf

Langhoff-Roos Jens

Langhoff-Roos Jens

Langhorne Peter

Langland-Orban Barbara

Lannin Natasha

Lansdorp-Vogelaar Iris

Lantos Hannah

Lao Chunhuan

Lao Li-Xing

Lao Terence

Lapin Brittany

Laplante-Levesque Ariane

Lara Elvira

Lareau Suzanne

Large Matthew

Larkin Philip

Larkins Nicholas

Larouche Richard

Larson Heidi

Larson Nicholas B

Larsson Matz

Larun Lillebeth

Lasalle Janine

Lash Jp

Lassen Ulrik

Lasserre Aurelie

Lasserson Daniel

Laszlo Brassai

Latif Mt
Latif Asam

Latini Roberto

Latter Sue

Lau Erica

Lauffenburger Julie

Launonen Ap

Lauridsen Jörgen

Lavelle Grace

Laver Dr Kate

Laver Kate

Laverty Anthony

Lavie Carl

Lavigne Mikael

Lavoie Kim

Lavoie Pascal

Lavorini Federico

Law Caroline

Law Matthew

Lawal Adegboyega

Lawesson Sofia

Lawlor Peter

Lawrence David

Lawrence Joseph

Lawrence M

Lawrence Sue

Lawrence Wendy

Lawrenson John

Lawrentschuk Nathan

Lawton Bev

Layton Natasha

Lazar Nicole

Lazarus John

Lazarus Lisa

Lazo-Langer A

Lazzara Elizabeth

Lazzarini Peter

Lazzeri Chiara

Lazzeri Massimo

Lazzerini Marzia

Lazzerini Pietro

Le Boutillier Clair

Le Dé Loïc

Le Guen Morgan

Le Meur Nolwenn

Le Roy Bertrand

Lea Richard

Leaf David

Lean Mike

Leas Eric C

Leavey Gerard

Leaviss J

Leblanc Marissa

Lebon Florent

Lebowitz Adam

Lecher Shirley

Lecky Fiona

Lee Ac

Lee Albert 
Lee Chang Hyun

Lee Christina

Lee Chung-Ta

Lee Edwin

Lee Elizabeth

Lee Eun-Young

Lee Guek Eng

Lee Gun Woo

Lee Hiu Man

Lee Hopin

Lee Hui-Jie

Lee Hyangsook

Lee Jae Ho

Lee Jt

Lee Ju Ah

Lee Jun-Hwan

Lee Jung-Hoon

Lee Kwan S

Lee Matthew

Lee Paul

Lee Peter

Lee Peter N

Lee Seungyoon

Lee Shaun Wen Huey

Lee Shen-Ming

Lee Shing Fung

Lee Shoo

Lee Shui Shan

Lee Siew

Lee $\mathrm{St}$

Lee Tien-Ching

Lee Tony Szu-Hsien

Lee Way Seah

Leeder Stephen

Leeflang Mariska

Leesar Massoud

Lefroy Je

Lega Ic

Legare France

Leger Damien

Lehert Philippe

Lehmacher Walter

Lehto Rebecca

Lehto Us

Leibowitz Ruth

Leino-Kilpi Helena

Leischik Roman

Leitao Sara

Leja Marcis

Lemak Christy Harris

Lemvik Grethe

Leng Sean

Lenhard Fabian

Lennon Marilyn

Lenoir-Wijnkoop Irene

Lensen Sarah

Lent $\mathrm{Mr}$

Leonard Maureen
Leonard S

Leone Jody

Leppakoski Tuija

Lerner J

Lerner $\mathrm{Nb}$

Lertxundi Unax

Leslie Hannah

Leslie Richard

Lesnierowska Magdalena

Lessard Laura

Lessells Richard J

Letilovic Tomislav

Leung Alexander

Leung $\mathrm{F}$

Leung Gk

Leung Janni

Leung Siu-Wai

Leuraud Klervi

Leutgeb Rüdiger

Levandowski Brooke

Levell Nick

Leventer-Roberts Maya

Levin Albert

Leviton Laura

Levy Jerrold

Levy Mark

Levy Ofer

Lewis Catrin

Lewis Gwyn

Lewis J

Lewis Jen

Lewis Joshua

Lewis Martyn

Lewis Mj

Lewis Myles

Lewis S

Lewis Sarah

Lexchin Joel

Leys Christophe

Li Alvin Ho-Ting

Li Changwei

Li Chao-Jui

Li Chiang-Shan

Li Chin-Shang

Li D-K

Li Dan

Li Emmy

Li Fan

Li Ho Cheung William

Li Jian-Jun

Li Jinke

Li L

Li Li-Ming

Li Ling

Li Mingyang

Li Qingfeng

Li Ru

Li Ruosha
Li Shelly-Anne

Li Sheyu

Li Xin

Li Xinjun

LiY

Li Yan

Li Yuanyuan

Liang Huey-Wen

Liang Fengxia

Liao S-Y

Liao Y

Liao Youlian

Liarski Vladimir

Liaw Jj

Liddy Clare

Lidón-Moyano Cristina

Lie Irene

Lie Sa

Liebeskind David

Liefers Gerrit-Jan

Liem Tk

Lievin-Le Moal Vanessa

Liew Danny

Liew Tau Ming

Ligthart Suzanne A

Lihana Raphael W

Lilford Richard

Liliemark Jan

Lillebo Børge

Lilly Christa

Lim Apiradee

Lim Chiao

Lim Kuang Hock

Lim Megan

Lim Seng-Gee

Lim Thiam

Lim Tow

Lim Wai H

Limburg Karina

Lin Chin

Lin Ching-Heng

Lin Elizabeth

Lin Gen-Min

Lin Haiqun

Lin Haotian

Lin Hualiang

Lin Jianchang

Lin Jianhao

Lin Junfen

Lin Kueiyu Joshua

Lin Lifeng

Lin Michael

Lin Michelle

Lin Ming-Chin

Lin Ning

Lin Tsung-Hsien

Lin Yan

Lin Yu Cih
Linaker Catherine

Lindbaek Morten

Lindberg Lene

Lindberg Magnus

Linde Klaus

Lindeman J H N

Lindemulder Susan J

Lindenmeyer Antje

Linder Oana

Lindert Jutta

Lindfors Olavi

Lindgren Kristen

Lindh Jonatan

Lindholm Bengt

Lindor Keith D

Lindqvist Pelle G

Lindsay Mk

Lindsey Laura

Linet Martha

Linhares Sâmia

Linsley Paul

Liotta Giuseppe

Lippert Freddy

Lipton Allan

Lisha N

Lisonkova Sarka

Lisowska-Myjak Barbara

Litonjua Augusto A

Litorp Helena

Litt Mark

Little Bertis

Little Jonathan

Little Julian

Littlechild Brian

Littman Alyson J

Litton Edward

Liu-Ambrose Teresa

Liu Aizhong

Liu Bin

Liu Bojing

Liu Cheng

Liu Chieh-Yu

Liu Cun-Zhi

Liu Dz

Liu Hao

Liu Jianmeng

Liu Kouying

Liu Lan

Liu Lei

Liu Li

Liu Michael

Liu Pei

Liu Qing

Liu Shijian

Liu Shuling

Liu X

Liu Xiaoyun

Liu Xiyang 
Liu Z

Liu Zhixin

Liu Zhiyong

Livingston Gill

Livingstone Shona

Livorsi Daniel J

Lizarondo Lucylynn

Ljung Rickard

Llopis Oscar

Llor Carl

Llovet Valentina

Lloyd-Sherlock Peter

Lloyd Cathy

Lloyd Jane

Lo Bernard

Lo Kristin

Lo Y L

Lobach I

Lobo Benjamin

Locatelli Francesco

Locatelli Igor

Lockey David

Loerbroks Adrian

Loewenstein David

Löf M

Løfgren Bo

Lofgren Niklas

Logan Robert

Logan Trevon

Loganathan Tharani

Logerstedt D

Logie Carmen

Loh Erwin

Loh Erwin

Lohman Matthew

Løkke Anders

Lokker Cynthia

Lokuge Buddhima

Lombard Carl

Lombardi F

Lomis Kd

Lonardo A

Lonardo Amedeo

Long Maggie

Longatto Filho Adhemar

Longstaff B

Looney Stephen

Lopes Luciane

López Gútiérrez Juan Carlos

López-López José

López-Ruiz María

Lopez Rodrigo

Lopez Violeta

Lophatananon Artitaya

Lord Rosemary

Lorden Andrea

Lorencatto Fabiana
Lorenz Eva

Lorenzetti Diane

Loring Stephen

Loten Conrad

Lotfi Tamara

Lotfi Vahid

Lotto Robyn

Lotz C

Loughlin John

Louie Av

Lourenço Óscar

Lous Jørgen

Loutfy Mona

Love Penny

Lovell Andrew

Lovqvist Chatarina

Lovrics Peter

Low Lian Leng

Lowe Jm

Lowe Nicola

Lowe Robyn

Lowenberg M

Lown Beth

Loy Veronica

Lozada Alfredo

Lu Ai-Ping

Lu Chuan-Jian

Lu Ciyong

Lu Esther

$\mathrm{Lu} \mathrm{Ht}$

$\mathrm{Lu} \mathrm{J}$

Lu Liming

Lu Xiang

Lu Y

Lu Ying

Lu Yun

Lu Yunxia

Lubrano Ennio

Luby Stephen

Luca Malcovati

Lucassen Peter

Lucchetti G

Lucenteforte Ersilia

Lucey Michael

Luck-Sikorski Claudia

Ludbrook Guy

Ludwig Michelle

Luedtke K

Lugon Jocemir Ronaldo

Luhmann N

Lui Chi-Wai

Luk Sabrina

Luker Julie

Lukersmith Sue

Lumbiganon $\mathrm{P}$

Lund Ingeborg

Lund Karl

Lundh Andreas
Lung For-Wey

Lunney June

Lunsky Yona

Lunyera Joseph

Lupattelli Angela

Luquiens A

Lurås Hilde

Lurie Jon

Lut Irina

Lutgens Danielle

Lutsar Irja

Luty Sue

Lutzhoft Margaretha

Ly Stephanie

Lycke Michelle

Lye David

Lyman Stephen

Lymperopoulos Anastasios

Lynagh Marita

Lynch Elizabeth

Lynch John

Lynch Katherine

Lynch Louise

Lynn Joanne

Lyon Alison

Lyons Karen

Lyons Zaza

Lystad Reidar

Ma C

Ma Guansheng

Ma Hon

Ma Hong

Ma Irene

Ma Jin

Ma Jun

Ma Michael

Ma Richard

Ma X

Maas Angela

Maas Esther

Maatouk Imad

Mabey David

Macaden Ashish

Macaluso Fabio Salvatore

Macarthur George

Macbeth Angus

Maccallum Peter

Maccarthy Sarah

Macdonald Alexander

Macdonald Jane

Macdonald Stephen

Macfarlane Alison

Machado-Alba Je

Machado-Rodrigues

Aristides M

Machado Gustavo

Macheiner Tanja

Macinnes Douglas
Macintyre Raina

Macisaac Richard J

Mack P

Mackenbach Joreintje

Mackenzie Adrian

Mackenzie Lynette

Mackey Dawn

Mackie Palmer

Macklin Ea

Macklon Nick

Maclaren Rob

Macleod Angus

Macleod M

Macleod Sheona

Maclure Katie

Macmanus Michael

Macmillan Freya

Macneela Padraig

Macpherson Miranda

Macpherson Peter

MacqJean

Mactier Helen

Madaj Barbara

Madas Balázs

Madden David

Madden Kenneth

Madden Kim

Madrid Eva

Maffulli Nicola

Maggio Lauren

Magin Parker

Magnavita Nicola

Magni Paolo

Magnusson Dawn

Magnusson Patrik

Magri Mariana

Magruder Kathryn

Maguire Aideen

Maguire Denise

Maguire K

Maguire Rebecca

Mahadeva Sanjiv

Mahajan Vikram

Mahapatra Tanmay

Maharajan Mari Kannan

Mahdy Abd Allah Heba

Mahenge B

Maher Chris

Maher Christopher

Mahmoud Elbattah

Mahon Susan

Mahoney Monica

Mahvu Webster

Mai Qun

Maidment Ian

Mailles A

Mainous Iii Arch

Maiorino Maria Ida 
Majmudar Maulik

Majtan Juraj

Makela M

Makker Himender

Makowski K

Makris Michael

Makumbi Frederick

Malafarina V

Malagon Talia

Malan L

Malan Zelra

Malara Alba

Malatino Lorenzo

Malcolm Dominic

Malemud Charles

Malički Mario

Malik Rayaz

Malik Vansanti

Malilay J

Malin Maili

Malin Sk

Malkin Robert

Malla Ashok

Mallia P

Mallineni Sk

Malmqvist Stefan

Malo S

Malone Helen

Malone Matthew

Malouf Reem

Maltezou Helena C

Malyar Nasser

Mamede Silvia

Mammone Alessia

Mamudu Hadii

Man Kwan

Manassiev Nikolai

Manavi Kaveh

Manchaiah Vinaya

Manchikanti Laxmaiah

Manchikanti Laxmaiah

Mancini John

Mancini Marcio C

Mandel Ellen

Mandelbrot L

Mandraffino G

Mandzia Jennifer

Manhart Lisa

Manias Elizabeth

Mann Steve

Mannan $\mathrm{H}$

Manning Jt

Manninger Martin

Mannino David

Mannino David

Mannion Russell

Mannucci Pm

Manolis Antonis S
Manor Orly

Manousaki Despoina

Manser Renee

Mansfield Avril

Mansfield E

Mansfield Louise

Mansfield Neil

Manske Robert

Manski-Nankervis Jo-Anne

Mansoor Ghulam

Mansour M

Mansournia Mohammad

Mansur Ashham

Mansur Rodrigo B

Mantadakis E

Manthey Jakob

Manyazewal Tsegahun

Manzo Ciro

Mao Jialin

Mao Ying Ying

Mao Zhiguo

Mapanga Witness

Mappin Bonnie

Marais Debbi

Marc Isabelle

March Sonja

Marcheselli Luigi

Marcías-Rodríguez

Ricardo

Marcinowicz Ludmila

Marckmann G

Marcovecchio Maria

Loredana

Marcu A

Marechaux S

Maret-Ouda John

Mariani Javier

Marijon Eloi

Marijuán Pc

Marik Paul

Marin-Hirsch Pierre

Marin Alicia

Marin Luz S

Marinacci Lx

Marinaccio Alessandro

Marino M

Mariotti Giuliano

Markevych Iana

Markham Sarah

Markovic Marija

Marks Angharad

Marks Florian

Marks Michael

Marks S

Marmamula Srinivas

Marmet Simon

Marom T

Marques Neves Carlos
Marques Roque Fatima

Marques-Vidal Pedro

Marques Adilson

Márquez-Magaña L

Márquez-Rodas Iván

Marra Carlo

Marroquin Bridget

Marsal K

Marsden John

Marsh Robert De Wilton

Marsh David

Marshall John

Marshall Nathaniel

Marshall Paul

Marshall Roger

Marshall S

Marshman Z

Marston Louise

Martel G

Mårtensson Lena

Martín-Guerrero José David

Martin-Lesende Inaki

Martín-Mateo Miguel

Martin-Moreno Jose M

Martin-Yuste Victoria

Martin Anne

Martin Anne

Martin Denis

Martin Elisa

Martin Emily

Martin Graham

Martin Jl

Martin Michael

Martindale Jenifer

Martinek Jan

Martinez De Tejada Begona

Martinez-Hernandez David

Martinez-Selles Manuel

Martinez-Vizcaino Vicente

Martínez M

Martins-Melo Francisco

Rogerlandio

Martins Cc

Martins Wellington

Martinusen Dan

Marto João Pedro

Martyn-St James Marrissa

Maruf Fatai A

Marui Akira

Marusic Ana

Maruta Jun

Maruyama Kenta

Maruyama Toru

Mas Miquel

Mascherini Gabriele
Maseda Ana

Maselko J

Masella Roberta

Mashamba-Thompson

Tivani

Mashige Khathutshelo

Percy Ercy

Maskarinec Gertraud

Maso Gianpaolo

Mason Amy

Mason Julia

Mason Linda

Mason Suzanne

Mass Andrew

Massad Salwa

Massey Suena

Masterson Siobhan

Masuet-Aumatell Cristina

Mata J

Matanovic Suzana Mimica

Matarma Tanja

Matata Bashir

Matcham Faith

Mate Karen

Mateu-De Antonio Javier

Mather Carey Ann

Mather Lisa

Mathes Tim

Matheson Catherine

Matheson David

Mathews Maria

Mathiesen Elisabeth R

Mathur Charu

Mathur Rohini

Matovu Joseph Kb

Matser Amy

Matsubara Shigeki

Matsuda Morihiro

Matsuyama Hideyasu

Matsuyama Tasuku

Matsuyama Yusuke

Mattar Rosiane

Matte Darlan

Mattei Peter

Matthews Anthony

Matthews Jessica

Matthews John T

Matthews Karen

Matthews T Wayne

Matthies Swantje

Mattner Jochen

Mattocks C

Matzer Franziska

Maura Géric

Mauss Daniel

Maust Dt

Mavilidi Myrto

Mavletova Aigul 
Mavragani Cp

Mavridis D

Mavrommatis Christos I

Maxim Paul

Maxwell Aj

Maxwell Margaret

Maxwell Rochelle

Mayanskiy Nikolay

Mayega Roy

Mayer Perry

Mayerl Hannes

Maynard Alan

Maynard Greg

Maynou Laia

Mayo-Wilson Evan

Mayor Eric

Mayr Astrid

Mayr Michael

Mayr Patrick

Mayrand Marie-Hélène

Mayrhofer Andrea

Mazinyo Ernesha

Mazzocato Pamela

Mazzocchi Mario

Mbah A U

Mbuagbaw Lawrence

Mbuagbaw Lawrence

Mc Hugh Sheena

Mcadams-Demarco Mara

Mcaleese Kirsty

Mcalinden Colm

Mcallister David

Mcallister Marion

Mcauliffe Linda

Mcbain H

Mcbride Craig

Mcbride David

Mcbride Mary

Mcbrien Kerry

Mccahon Deborah

Mccain R

Mccallum Debra

Mccann Edward

Mccarthy Elaine

Mccarthy Jane

Mccarthy Lisa

Mccarthy Noel

Mccarthy Ona

Mccarthy Robert

Mccartney $\mathrm{Gj}$

Mccartney Gerry

Mccaul Conan

Mcclelland Jodie A

Mccloskey Eugene

Mccloskey Eugene

Mccloskey Rose

Mcclure Elizabeth

Mcclurg Doreen
Mccollum Eric

Mcconkey R

Mcconnachie Alex

Mccool Judith

Mccracken Lance

Mccreedy Ellen

Mccreesh Karen M

Mcculloch Charles

Mcculloch Peter

Mcculloh David

Mccullough Hazel

Mccullough L

Mccullough Mac

Mccullough Marji

Mccully Kilmer

Mcdermott Clare

Mcdonagh Marian S

Mcdonald John

Mcdonald John H

Mcdonald Scott

Mcdonald Suzanne

Mcdonald Timothy

Mcdonnell Mj

Mcdonough Suzanne

Mcdougall Catherine

Mcdowell Michelle

Mcelhaney Janet

Mcewan Desmond

Mcfarland Agi

Mcfarland Dm

Mcgaraughty Steve

Mcgeechan Kevin

Mcgiffin Jed

Mcginnes Rosemary

Mcginty Emma

Mcginty Ee

Mcgovern Andrew

Mcgowan Kelly

Mcgrath Deirdre

Mcgready Rose

Mcgregor Gordon

Mcgregor Jules

Mcguinness Myra

Mcguire Joseph F

Mcgwin Jr Gerald

Mchugh Lisa

Mchugh Marry

Mcintosh Scott

Mcintyre Meredith

Mcivor Gill

Mckeague Ian

Mckechnie Jacqui

Mckee G

Mckeever Robert

Mckenzie K

Mckevitt Christopher

Mckinlay Audrey

Mckinnon L
Mckinstry Brian

Mclachlan John

Mclain Alexander C

Mclellan Mary C

Mcleod Charlie

Mcleod Donald

Mcleod Graeme

Mcluckie Alan

Mcmahon EM

Mcmanus Chris

Mcmanus Richard

Mcmaster Fiona

Mcmeekin Peter

Mcmillan Tom

Mcnab Justin

Mcnally Richard

Mcnamara Kevin

Mcnaughton N

Mcnaughton Nancy

Mcnaughton Rebekah J

Mcneil John

Mcnicol Ewan

Mcnulty Cliodna

Mcphee Jamie

Mcpherson Amy

Mcpherson Elizabeth

Mcpherson Stuart

Mcroy Susan

Mcshane Helen

Mcshane Kelly E Mcshane

Mcsharry Jenny

Mcvicar Andrew

Mcwilliams Dan

Meacock Rachel

Meade Elizabeth

Meader Nick

Meaney Sarah

Mearns Gael

Mearns Kathryn

Mebazaa Alexandre

Meccariello Giuseppe

Medeiros Daniela

Meehan Elaine

Meeks Karlijn

Meena Kumari K

Meganck Reitske

Meggi B

Meggiolaro Angela

Mehlum Ingrid

Mehrotra A

Mehrotra Ravi

Mehrpour Omid

Mehta Bella

Mehta Supriya

Mehtry Vijay

Mehuys Els

Meier Christoph R

Meijer Chris
Meiklejohn Sarah

Meil William

Meireles Paula

Meiring Rm

Meiser Bettina

Meissner Konrad

Mejia Anilena

Mejia Luis F

Mejri Atef

Mekary Rania

Melberg Andrea

Melbye Hasse

Melchiorre Maria

Gabriella

Melendez-Torres GJ

Melendrez Melanie

Melgaard Bjorn

Melkonian Stephanie

Mello Johanna De

Almeida

Menasche Philippe

Mendez-Bailon Manuel

Menec Vh

Meneghini Michael

Meneses-Echavez Jose

Meng Xingqiong

Menguer Rodrigo

Mennemeyer St

Menni Cristina

Menon Bk

Menon Sonia

Menzaghi C

Menzies Robert

Merat Shahin

Mercer Judith

Mercer Stewart

Merchant Reshma

Mercier Eric

Merkel S

Merlo Lisa

Merriel Samuel

Merritt Robert

Merry S

Mertens Peter

Mesarwi Omar

Meshkini M

Meshram Indrapal

Messerli Franz

Mestres Conxita

Metcalf Patricia

Metcalfe Amy

Metcalfe David

Meteran Howraman

Methley Abigail

Metlaine Arnaud

Metsvaht Tuuli

Meulemeester Kayleigh

Meurer William 
Meurman Jukka

Meybeck A

Meyer-Baron Monika

Meyer Anne

Meyer Beau

Meyer Carly

Meyer Pamela

Meyer Thorsten

Meyfroidt Geert

Meyhoff Christian

Meynaar Ia

Meyre David

Meyrose Ann-Katrin

Meysamie Alipasha

Mi M

Miake-Lye Isomi

Miani Celine

Miao Li-Yan

Miceli-Richard Corinne

Miceli Luca

Michael Dunne

Michaleff Zoe

Michalski Christoph

Michaud Dominique

Michel Jean-Baptiste

Middlekoop Keren

Middleton Jo

Middleton Philippa

Midori Castelo Paula

Miedema Baukje

Mielenz Tj

Migyanka Joann

Mihala Gabor

Mihrshahi Seema

Mikhailova Alexandra

Mikkelsen M

Mikolajczyk Adam E

Mikolajczyk Rafael

Milani Daniela

Milani Richard

Milaniak Irena

Miles Lachlan

Millan-Guerrero Rebeca O

Miller Andrew

Miller Charles

Miller Lyndsey M

Miller Robin

Millner Alex

Mills-Finnerty Colleen

Mills Kathryn

Milne Barry

Milner James

Milo R

Milsten Andrew

Miltenburg Andrea

Miltz Ada

$\mathrm{Min} \mathrm{Su}$

Minabe Yoshio
Minary Laetitia

Mindry Deborah

Miner Brienne

Miniati M

Minihan Paula M

Minkowitz Barbara

Minnie Karin

Minton Jonathan

Mintzes Barbara

Mion Lorraine

Mirabelli Carmen

Miraglia Mariella

Miranda-Mendizabal

Andrea

Miranda Angelica

Mirzazadeh Ali

Misery Laurent

Mishara Brian

Mishra Devendra

Mishra Anil

Mishra Si

Mishriky Adel M

Misra Ravi

Misra Satyajeet

Misset Benoit

Mistry Pankaj

Mitanchez Delphine

Mitchell Ae

Mitchell Paul

Mitchell-Jones Nicola

Mitchell Aaron

Mitchell Caroline

Mitchell Colin

Mitchell Elizabeth

Mitchell Patrick

Mitchell Sarah

Mithra P

Mitra Biswadev

Mitra M

Mitra Monika

Mitra Saikat

Mitra Sukanya

Mitsch Andrew

Mittal Srabani

Mittlbock Martina

Miura Fumihiko

Miura Kyoko

Miyamori Daisuke

Miyamoto Keiko

Miyata Masaaki

Miyazaki Jun

Miyazaki Mariko

Mjøen Geir

Mlynarska Agnieszka

Mo Xiaokui

Mobbs Ralph J

Mockel Martin

Mockford Carole
Modesti Pietro

Modig Karin

Moeller Jette

Moen Be

Mogre Victor

Mohaghegh Shalmani H

Mohamad Noor Z

Mohamed Ne

Mohammed Kahee

Mohan V

Mohanty Sanjay

Mohanty Sk

Mohd Yusof Barakatun

Nisak

Mohebbi Mohammadreza

Möhler Ralph

Mohr Oliver

Moini Ashraf

Moise Nathalie

Moisi Marc

Mokkink Lidwine

Mol Ben

Mol Mayke

Molassiotis Alex

Molcho Michal

Molina Gf

Molinari Noelleangelique $\mathrm{M}$

Moll Henriëtte

Moll Rachel

Molle Roberta

Møller Tp

Møller Morten

Möller Riitta

Molto Anna

Mombo-Ngoma Ghyslain

Monaghan Nigel

Monahan Kevin

Monami Matteo

Moncada-Jimenez Jose

Moncrieff Joanna

Mondillon Laurie

Mondul Alison

Moniruzzaman

Mohammad

Montag Annika

Montagnese Thomas A

Montalto Michael

Montano-Loza Aldo

Montano Adriana

Monteiro S

Montelpare William

Montes-Torres Julio

Montez-Rath Maria E

Montgomery Antony

Montgomery Jennifer

Montgomery Jenny

Montgomery Scott
Moodie Crawford

Moodley J

Moodley Jennifer

Moodley Y

Moon Heehyul

Moor Molly

Moore Andrew

Moore Chris L

Moore Justin

Moore Nicolas

Moore Simon

Moores Thomas

Moosa Shabir

Moosazadeh Mahmood

Moppett Iain

Mor Orna

Mora Jaume

Morais Rui

Moraleda Cinta

Moran Alan

Moran Andrew

Moran Chris

Moran Lisa

Moran Valerie

Morandi Alessandro

Moranne Olivier

Morano Jamie

Mørch Lina

Moreira-Marconi Eloa

Moreno Santagio

Moreton Adam

Morey Brittany N

Morgan Alison

Morgan Jake

Morgan Philip

Morgan Sarah

Morgan Steve

Morgan Vicky

Mori Masanori

Moriarty Frank

Morikane Keita

Morina David

Moritz Ml

Mork Bjorn

Morken Nils-Halvdan

Mørkrid Lars

Morley Christopher P

Morley Katherine

Moro Pedro L

Moro Ruth

Morphett Kylie

Morris C

Morris Joanne

Morris Jonathan

Morris Julie

Morris Keith

Morris Luc Gt

Morris Melanie 
Morris Reg

Morris Theresa

Morrison Val

Morriss Richard

Morsy R

Mort Maggie

Mortensen $\mathrm{H}$

Mortensen J

Mortenson Ben

Morton Adam

Morton Dion

Morton Jeremy

Morton Katherine

Morton Patricia

Morton Robert

Morville Anne-Le

Mosby Danielle

Mosca Antonella

Mosca Ms

Moscoso Miguel

Mosdøl Annhild

Moser Asher

Moshammer Hans

Mosler Hans-Joachim

Moss Brian

Mosweu Iris

Motamed N

Motegi Sei-Ichiro

Moth Grete

Motov Sergey

Motta-Castro Ana Rita

Motta Francesco

Mou Yi

Moum Torbjørn

Movilla Fernández María Jesús

Movsisyan N

Mowafi Hani

Moy Marilyn L

Moynihan Melissa

Moynihan Ray

Moynihan Ray

$\mathrm{Mu}$ Lin

Mudge Alison

Muehlschlegel Susanne

Mueller-Stierlin Annabel

Sandra

Mueller Martin

Mueller Ps

Muffly Matthew

Muggah Elizabeth

Mugo Ngatho

Muhardi Leilani

Mühlhauser Ingrid

Muhm M

Muhoozi Grace

Muhsen Khitam

Muilwijk Mirthe
Muir-Hunter Susan W

Muir Keith

Mujika Agurtzane

Mukadam Naaheed

Mukerji Geetha

Mukherjee Mome

Mulder Roger

Mulders-Manders C

Mulick Cassidy Amy

Mulkey M

Muller Vahl Kirsten

Müller Andre

Müller Christian

Müller Ulrich

Mulley William

Mulligan Hilda

Mullin Daniel

Mullins Jamie

Mulraney Melissa

Mulu Andargachew

Mulwafu W

Mumtaz Zubia

Munce Sarah

Munford Luke

Munk Heidi

Munnangi S

Muñoz Manolo

Munson Erik

Münster-Halvari Anne

Murad Fadi

Murad M Hassan

Murakawa Yuji

Murcia Mario

Murcott William

Murdoch Jamie

Murdock Kyle

Murgatroyd Darnel

Murgic Jure

Murhekar Manoj

Murki Srinivas

Murnane Elizabeth

Murphy A

Murphy Adrianna

Murphy Catherine

Murphy Dominic

Murphy Hj

Murphy Joseph

Murphy Margit Alt

Murphy Michael

Murphy Mike

Murphy Patrick

Murphy William

Murray Christina

Murray David

Murray Deidre

Murray Greg

Murray Marylou

Murray Sarah
Murthy Sb

Murthy Srinivas

Muscara F

Muscat Dm

Musekiwa Alfred

Musey Paul

Musinguzi Geofrey

Musser Erica D

Mustafa Hesham

Mustafa Yassar

Mütsch Margot

Mutz Michael

Muzumdar Radhika

Mwaka Amos

Mwirigi Anne

Mwita Julius

Myall Michelle

Myers Jamie

Myers Michael

Myerson Rebecca

Myhre Peder

Myint Tun

Myles Paul

Mylona Kalliopi

Myntti Tarja

Myong Jun-Pyo

Mystakidou Kyriaki

Mytton Oliver

Mzayek F

Núñez Julio

Naaktgeboren Ca

Naber Kurt

Nabhani-Gebara Shereen

Nabi Hermann

Nabil Wan

Nabulsi Mona

Nabwera Helen

Naci Huseyin

Nacul Luis

Nădăşan V

Nadel Simon

Nadia Dimitrova

Nadkarni Abhijit

Nadkarni Vinay

Nadorff Michael

Naess Halvor

Nafstad Per

Nagahama Satsue

Nagai Hideaki

Nagai Masato

Nagai S

Nagarajan Neeraja

Nagashima Akimichi

Nagata Chie

Nagel Eike

Nagel Maria A

Nah Eun-Hee

Nahar Vk
Naidoo Datshana

Naiem Ahmed

Nair Manisha

Nair Rajeshwari

Najafipour Hamid

Najjar Shahenaz

Nakada Haruka

Nakagawa Naoki

Nakamura Priscila M

Nakamura Kazutoshi

Nakamura Mieko

Nakamura Sayaka

Nakamura Sunao

Nakamura Takehiro

Nakamura Tomoki

Nakanishi Miharu

Nakase Hiroshi

Nam Hae-Sung

Nambiar Anoop

Nana Djeunga Hugues

Clotaire

Nanchahal Kiran

Nanchen David

Nandy Rajesh

Nanton Veronica

Nap Alexander

Narang A

Naranjo Mario

Narasimhan Padmanesan

Narod Steven A

Nasci Bruno

Nascimento-Carvalho

Cristiana

Nash Louise

Naslund John

Nassar Dua' Abdellatef

Nassif Hala

Natafgi Nabil

Natale Andrea

Nathanson David

Naudet Florian

Naughton Corina

Naugler Christopher

Navarro-Ledesma Santiago

Navarro Enrique

Navarro Robert

Navas-Carretero Santiago

Naveh Eitan

Nayoan Johana

Nazar Gaurang

Nazar Hamde

Nazare Bárbara

Nazmi Aydin

Ncayiyana Jabulani

Ndejjo Rawlance

Nebot César

Nee Patrick A

Neef Hannes 
Negatu Beyene

Negoi Ionut

Negro Roberto

Nehlin Christina

Nehme Ziad

Neidert Mc

Neill Sarah

Nelson Amanda

Nelson E Andrea

Nelson Eugene

Nelson Fred R

Nelson G

Nelson Gregg

Nelson Heidi

Nelson Jon P

Nelson Peter

Nelson Sarah E

Nelson Stephen

Nemery Benoit

Nemes Szilard

Nenna Raffaella

Neophytou Andreas

Neri Luca

Ness Ottar

Nettleton Sarah

Neubeck Lis

Neuberger James

Neuberger Manfred

Neuerburg Carl

Neufeld Eva

Neuhaus Christopher

Neupane Subas

Neupane Subas

Nevill Alan

Neville Stephen

New Pamela

Newberry Jennifer

Newberry Sydne

Newby David

Newton-John Toby

Newton Nicola

Newton Tim

Neyra Ja

Ng Fat Linda

Ng Winda

Ngari Moses

Ngarina Matilda

Nglazi Mweete

Nguyen T

Nguyen Huong

Nguyen Ly

Nguyen Phan

Nguyen T

Nguyen Van Thi Thuy

Ni Melody

Nian Hui

Nichol Graham

Nicholas J
Nicholls Rachel

Nicholls A

Nicholls Stuart

Nichols Linda

Nicholson Sandra

Nici Linda

Nickel Christian

Nickel Brooke

Nicolaou M

Nicolson Philippa

Niederman Ms

Niehaus William

Nielsen Anette

Nielsen Bibi

Nielsen Karoline

Kragelund

Nielsen Peter

Nielsen Sabrina

Nieminen Pekka

Nieuwenhuis J

Nieuwkerk Pythia

Nigenda Gustavo

Nijman R

Niklasson Mats

Nikolic Igor

Nilsen Wendy

Nilsson Evalill

Nilsson Hanna

Nilsson Lena

Nilsson Lennart

Nilsson Peter

Nimegeer Amy

Ninomiya Toshiharu

Nirantharakumar

Krishnarajah

Nisar Yasir Bin

Nishi Nobuo

Nishida Yuichiro

Nishikawa Masatomo

Nishioka Shinta

Nishiwaki Masato

Nishiyam Tsutomu

Nitkunan Arani

Nitsch Dorothea

Nixon Elena

Njoroge Martin

Noah Norman

Noakes Timothy David

Noar Seth

Noble Helen

Noble Jm

Nochioka Kotaro

Noelker Joan

Noertjojo Kukuh

Nogueira Cortez Daniel

Nohara Atsushi

Noheria Amit

Noipayak Pongsak
Noone P

Noppens Ruediger

Norcini John

Nordberg Gunnar

Nordenskjöld Jesper

Nordin Maria

Nordstrom Tobias

Norman Gill

Norman Paul

Norström Fredrik

Northwood Melissa

Norwood Patricia

Nossent Johannes

Notara V

Notaras Michael

Notcutt Willy

Notenboom Kim

Nothelle Stephanie

Nourbakhsh Bardia

Nouri Aria

Novaes H

Novak Laurie

Novovic $\mathrm{S}$

Nowalk Mary Patricia

Nowossadeck E

Noyez Luc

Nsuami M Jacques

Ntani G

Ntuli Sam

Nuel G

Nugent Allison

Nugent Kevin

Nugent Linda

Numans Mattijs

Nunes Anthony

Nunes José Pedro L

Núñez-Córdoba Jorge M

Nurmikko T

Nurmohamed Mike

Nutbeam Don

Nuzzo Regina

Nwana Nwabunie

Nyangoma So

Nylenna Magne

Nyquist F

Nyström Monica

Caoimh Ronan

O'brien Kate

O'brien Kelly

O'brien Sarah H

O'callaghan Clare

O'cathain Alicia

O'connell Matthew

O'connell Megan

O'connor Elizabeth

O'connor Richard

O'connor $\mathrm{S}$

O'connor Siobhan
O'donnell Aisling

O'donnell Catherine

O'driscoll Ronan

O'dwyer Maire

O'flynn Norma

O'gara Erin

O'grady Gregory

O'hara Nathan

O'higgins James

O'keeffe Colin

O'leary Jd

O'mara Da

O'neill Sean M

O'reilly Seamus

O'sullivan Dympna

O'sullivan Esther

O’Brien Anthony

Oakes D

Oakley Laura

Obaro Stephen

Ober Allison

Ocal Pelin

Oconnor Rod

Oda Eiji

Odei James

Odejinmi Funlayo

Odekerken $\mathrm{Vj}$

Oderkirk Jillian

Odone A

Odonnell Colm

Odunaiya N

Oertelt-Prigione Sabrine

Offiah Amaka

Ofori Sandra

Oga Toru

Ogaji Daprim

Ogawa Yukari

Ogden Terje

Ogilvie R

Ogino Shuji

Ogliari G

Ogundele O

Ogunmola Olarinde J

Ogunniyi Adesola

Oh Byeongsang

Oh Cheongeun

Oh Cheongeun

Oh Juhwan

Ohara Tomoyuki

Ohishi Mitsuru

Ohishi Mitsuru

Ohkuchi Akihide

Ohno-Matsui Kyoko

Ohno Yoshio

Ohrvik John

Ohta M

Ohtonari Tatsuya

Öien Rut 
Oiestad B E

Oiye Shadrack

Ojala Tapio

Ojeda Soledad

Ok Ercan

Okan Orkan

Okazaki Ryo

Oke Jason

Okeke In

Okello Emmy

Okello Samson

Okpechi Ikechi G

Okubo Yusuke

Okuno Yuji

Okuyama Ayako

Okyay Ramazan

Ola Bolanle

Olalekan Ayo-Yusuf

Olander Elinor

Olaniran Abimbola

Olaniyan T

Olayiwola J

Oldroyd John

Olds Margie

Olds Timothy

Olesen Anne

Oleson Stephanie

Oliveira De Melo Daniela

Oliveira Carlos

Oliveira EA

Oliveira J

Oliveira Luis Vicente

Oliveira Ma

Oliveira Rui

Oliveira Vinicius

Oliver Emily

Olives Travis D

Olivier Jake

Oloughlin Jennifer

Olovsson M

Olsen Kirsten

Olsen Sonja

Olshansky B

Olshen Richard

Olson Robert

Olsson Cecilia

Olsson Daniel

Olszewski Adam J

Olumuyiwa-Akeredolu Oo

Omae Kenji

Omar Rumana

Ombelet Willem

Omboni Stefano

Onakpoya Igho

Onakpoya Igho

Onder G

Onder Graziano

Onerup Aron
Ong Cheung-Ter

Ong Jason

Ong Tc

Oo Mt

Ooms Linda

Oostenbrink Rianne

Oosterman Joukje

Oppenheim Beryl

Oppikofer Sandra

Oppong Ray

Or Calvin

Oral Evrim

Oram Sian

Orandi Bj

Orchard Treena

Ordonez Mena Jose Manuel

Ordóñez-Mena Jm

Oreilly Dermot

Oremus Mark

Oremus Mark

Orimo H

Orisakwe Orish

Orri Massimiliano

Orsi Chiara

Ortiz Arduan

Ortquist Ake

Ortu Andrea

Orueta Juan

Osborn $\mathrm{Ha}$

Osborne Richard

Osborne Sonya

Osborne Thomas R

Oshitani Hitoshi

Oskoui M

Osma J

Osrin David

Oster Matthew E

Oster Richard

Osteras Nina

Østhus S

Ostini Remo

Otero A

Othman Nor

Ottenheijm Ramon

Ou Judy

Ouchi Shohei

Oudijk Martijn A

Ouellette-Kuntz H

Oulhote Y

Oulton Kate

Ouyang Bichun

$\varnothing$ verby Nina Cecilie

Overend $\mathrm{T}$

Overgaard M

Overgaard Søren

Ovesen Christian

Ovesen Therese
Oviedo-Trespalacios Oscar

Ovseiko Pavel

Owen Alice

Owen Neville

Owens David

Owens La

Owusu-Dabo Ellis

Oya Mototsugu

Oyo-Ita Angela

Ozakinci Gozde

Ozawa Tsuyoshi

Oze Isao

Ozen Nurten

Ozery-Flato Michal

Ozgu-Erdinc As

Ozgur K

Ozkaya Enis

Ozkiraz Servet

Ozturk Onur

Pääkkönen Markus

Pac Agnieszka

Paccione Gerald

Pacheco Joseph

Pacheco Julianna

Pacquiao Dula

Padayachy Lc

Padayatchi N

Padmakumar A

Padua Luca

Padula Rs

Padwal Raj S

Paez Arsenio

Pagaiya Nonglak

Pagán Ja

Paganoni Sabrina

Page-Shipp L

Page Amy

Page Christian

Page Geoff

Page M

Page Matthew

Pai Yogesh

Paige Ellie

Paige Ellie

Paige John T

Paige Samantha

Painchaud Guérard

Geneviève

Painter Rebecca

Pajak A

Pajno Giovanni

Pal Jd

Palabiyik Figen

Palacios-Cena Domingo

Palaniswamy Saranya

Palau Patricia

Palazón-Bru Antonio

Palazzo Francesco
Palen Ted E

Palese Alvisa

Paley Carole

Palinkas M

Paljarvi Tapio

Palla Mohan

Palladino Raffaele

Palm Rebecca

Palmer Andrew

Palmer T

Palmier-Claus Jasper

Palmieri Vittorio

Palombi Leonardo

Palta Mari

Pan An

Pan $\mathrm{H}$

Pan Sai

Pan X

Pan Xi

Panay Nick

Panchal Hemang

Panczyk Mariusz

Panda Rajmohan

Pandey Arvind

Pandey Kiran

Pandian Jeyaraj

Pandis Nikolaos

Pandit Anil

Pandit Hemant

Panduro Arturo

Pandya Amit

Panegyres Peter

Pang Marco

Pani Sharat

Panicker Vijay

Paniello Randal C

Pannequin J

Pant Puspa Raj

Pantic Igor

Panza Francesco

Panza Julio

Paolino Melisa

Paotonu Dianne

Papadakaki Maria

Papadimitriou C

Papadimitriou Lampros

Papageorgiou Sn

Papaioannou Andriana

Papamichalis $\mathrm{P}$

Papandonatos George

Papandonatos George D

Papanicolas Irene

Papoutsakis C

Papoutsi Chrysanthi

Papoutsis Dimitrios

Parameswaran Rajeev

Paranhos L

Paranjothy Shantini 
Paratz Jennifer

Paravattil Bridget

Parazzi Pl

Pardo-Hernandez Hector

Pardo Alba

Parenti Nicola

Parienti Jean-Jacques

Parikh Ravi

Parikh Sv

Paris J

Park Boyoung

Park Chanhyun

Park Eun-Cheol

Park Ji Won

Park Jong Hyock

Park Sang O

Park Seo Young

Park Yung

Parker Adwoa

Parker Martyn

Parker Rb

Parker Richard

Parker Shefton

Parkes Alison

Parkes Matthew

Parkin Lianne

Parle James

Parlesak Alexandr

Parmar Priya

Paro Helena

Parpia Sameer

Parry Gareth

Parry Neil

Parsons Carole

Parsons Judith

Parsons S

Partington Andrew

Partridge Stephanie

Parvez Sarker Masud

Pascual Julio

Paskins Zoe

Pasquier Gilles

Passalacqua Giovanni

Passchier Jan

Passmore Hayley

Passot G

Pasterkamp Gerard

Pasternak Bjorn

Pastore Ca

Pastori Daniele

Patakia Sonak

Patel Kunal

Patel Arpan

Patel Kanan

Patel Kishan

Patel Mehul D

Patel Rashmi

Patel Ronak
Patel Shivani

Paterson Abby

Paterson Craig

Paterson Ian

Paterson Nicole D

Paterson Pauline

Pathak Mona

Pathan Nazima

Pathania Deepak

Pathirana Induruwa

Patil Naeem K

Patja Kristiina

Paton Julian

Patrikios Ioannis S

Patterson Chris

Patterson Linda

Patterson Sue

Patton R

Pattussi Mp

Paudyal Priyamvada

Paul-Ebhohimhen Va

Paul Dinesh

Paul Friedemann

Paulsen Aj

Paulson-Karlsson Gunilla

Paulus Dj

Pauly Bernadette

Pavasini Rita

Pavkov Me

Pavlova Milena

Pavy B

Pawlak Maciej

Paz-Bailey G

Pazos M

Pears Sally

Pearse Rupert

Peate Michelle

Peck Kyong

Peden Amy

Pedersen A

Pednekar M S

Pedone Claudio

Pedrazzani Corrado

Peek-Asa Corinne

Peel Nancye

Peh Hong Yong

Peixoto Bruno

Pekmezovic Tatjana D

Pelikan Jürgen

Pellatt-Higgins T

Pelliccia Francesco

Pellicciari Leonardo

Pellicori Pierpaolo

Peloso Pm

Pemberton Chris

Pembrey Marcus

Peñate-Castro Wenceslao

Pendlebury Sarah
Pendlebury Sarah T

Penfold Chris

Penfold Robert

Peng Liubao

Peng Tao

Peng Yinan

Pengcheng Xun

Penn Anthony

Penn Claire

Penn Linda

Pennington David

Pennington Mark

Penno G

Pentzek M

Pepe J

Perazzo Joe

Pereira Da Silva José

António

Pereira Gray Denis

Pereira Dora

Pereira JC

Perera Manuja

Pérez Jolles Mónica

Pérez-Cambrodí Rafael José

Pérez-Hoyos Santiago

Perez-Martinez $\mathrm{P}$

Pérez-Zepeda Mario Ulises

Perez Antonio

Perez David Lewis

Perez Hector

Pergialiotis V

Pericas Juan

Perico Norberto

Perimal-Lewis L

Perkins Bruce

Perlman Sharon

Perlman Sharon

Perna Annalisa

Perovic Olga

Perrenoud Patricia

Perrier E

Perrier Laure

Perrin Peggy

Perry Daniel

Perry Hb

Perry Ivan

Perry Yael

Persoskie Alexander

Pesquero Joao Bosco

Pesut Barbara

Peter-Derex Laure

Peters Cheryl

Peters Edgar

Peters MJ

Peters Micah

Peters Remco

Peters Sanne
Petersen Irene

Petersen Reneva

Peterson Catherine A

Peterson Emily

Peterson Gregory

Peterson Kim

Petit Audrey

Petkus Andrew

Peto Tunde

Peto Tunde

Petousis-Harris Helen

Petousis Stamatios

Petráš Marek

Petre Benoit

Petrenko Christie

Petri William A

Petroff David

Petrova Anna

Petrova Mila

Petrucciani Niccolò

Petruzziello Arnolfo

Petty Duncan

Pevnick Joshua M

Peyré-Tartaruga Leonardo

Pezzilli Raffaele

Pezzini Alessandro

Pfaff Colin

Pfarrwaller Eva

Pfeiffer Michaela

Pfortner Timo-Kolja

Pham Minh

Phan Phillip

Phelan Amanda

Philbin Morgan M

Philbrook Le

Philip Femi

Phillips-Howard Penelope

Phillips Alexander

Phillips Anna C

Phillips Ceri

Phillips Christine

Phillips Craig L

Phillips David

Phillips Jg

Phillips Patrick

Phillips Robert

Philpott Matthew

Phinney Stephen D

Phipps Denham

Phuka John

Piatt Joseph H

Pibarot Philippe

Piccini Jonathan

Piccinni Carlo

Picco Louisa

Piccoli G B

Pickard Susan

Pickering Gisèle 
Pickering Ruth M

Pickett Kate

Pickett William

Pickles Michael

Pickles Timothy

Piekoszewski Wojciech

Pienaar Elizabeth

Pierrakos Charalampos

Pietruszka Barbara

Pietrzyk Lukasz

Piirtola Maarit

Pikala Małgorzata

Piler P

Pillai Avinesh

Pilling Luke

Pilling Rachel

Pilote Louise

Pilotto Sara

Pimentel Mauricio

Pincus Tamar

Pincus Theodore

Pinder Richard

Pinheiro T V

Pinkerton Kent E

Pinnix Cc

Pintello Denise

Pinto-Sietsma Sara-Joan

Pinto Nelangi

Pintye Jillian

Piovani Daniele

Piovezan Ronaldo

Piper Amanda

Piper Rory J

Pires Sara

Pirracchio Romain

Pisanelli Giovanni

Pisegna Jessica

Pistilli Maxwell

Pita-Fernandez S

Piterman Leon

Pitman Alexandra

Pitt B

Pittiruti Mauro

Pittrof R

Pitts Maggie

Pitts Stephen

Piza Caio

Plagerson Sophie

Plancher Kevin

Plank-Bazinet Jennifer

Plans-Rubio Pedro

Platt Mj

Platts-Mills Tf

Platts Loretta G

Plumb James

Plummer Virginia

Plunkett Adrian

Plusquin M
Plym A

Poder Thomas

Podlekareva Dn

Poethko-Mueller C

Pogge Elizabeth

Poggio Rossana

Pohontsch Nadine

Pol Ra

Poldervaart Judith

Polin Richard

Polisena Julie

Polivka Barbara

Pollesello Piero

Polsky Jane

Poluzzi Elisabetta

Polverino F

Pomeroy Am

Pompeii Lisa

Pompili Maurizio

Pond Dimity

Pong Raymond

Ponizovsky Alexander M

Pons-Vigués Mariona

Ponsioen Cyriel

Pont Lisa

Pontiroli Antonio

Pontisso Patrizia

Poole Dn

Poole Phillippa

Poovorawan K

Pop Ioana Andreea

Pope Catherine

Popejoy Emma

Popescu I

Poppe K

Popper Helmut

Porrata Luis F

Porta Miquel

Porte Robert

Portelli Jonathan

Porter Ian

Poss Jeff

Post Rm

Postma Douwe F

Postma Maarten

Postma Maarten

Postoev Va

Potaris K

Potdar Pd

Pothirat Chaicharn

Potier Tara

Pottegard Anton

Potter Shelley

Potvin Louise

Poulton Sally

Pourhoseingholi

Mohamad

Pourmarzi D
Pouwels Koen B

Pouwels Xavier

Povalej Petra

Powell Byron J

Powell Janet

Pradhan Bandana

Prado Elizabeth

Prados-Torres Alexandra

Präg Patrick

Prandoni Paolo

Prasad V

Prasad V

Prasetyo Aa

Prashanth Ns

Pratt Nicole

Pratt R

Prazeres Filipe

Precioso J

Preisser J

Prendergast Luke

Prentice Sarah

Prescott Gordon

Prescott Hallie

Prescott Robin

Preston Jenny

Preston Nick

Pribicevic M

Price Gill

Price Je

Price Lisa

Price M

Price Matthew A

Pridemore William

Prieto-Alhambra Daniel

Pringsheim Tamara

Priolo-Filho Sidnei

Pripp Are

Pripp Are Hugo

Proctor Susan P

Proietti Marco

Proietto J

Proost Johannes

Prosperini Luca

Prospero Emilia

Protiere Christel

Proudlove Nathan

Provencher Véronique

Provenzi Livio

Prue G

Prus Steven

Pruszkowska-Przybylska Paulina

Pryce J

Pryjmachuk Steven

Przybyla Jennifer

Przysucha Ep

Psallidas Ioannis

Puddu Paolo
Pudney Steve

Pudwell Jessica

Puente-Maestu Luis

Pugliese Giuseppe

Puigpinos Rosa

Pujol Miquel

Pulcini J

Puliyel Jacob

Pullicino Glorianne

Punamaki Raiija-Leena

Purepong Nithima

Purgato Marianna

Puri Basant

Puschner Bernd

Puthucheary Zudin

Puthussery Shuby

Puts Martine

Pyeritz Reed

Qato Dima

Qi Wenjing

Qi Xingshun

Qian Wenwei

Qin Wei

Qin Yanwen

Qingyue Meng

Qiu Chengxuan

Qixiao Jiang

Quader Zs

Quaife Matthew

Quan Hude

Quantin Catherine

Quarles Darryl

Quenot Jean-Pierre

Quesada-Gómez C

Quince Thelma

Quinn Megan

Quinn Terry

Quispe R

Quon Bradley S

Qurashi I

Qureshi Nadeem

Quyn Aaron

R Babu Giridhara

Raaff Carol

Rabbani Hossein

Rabbat Mark

Rabbitt Sarah

Rabin Elaine

Rabkin SW

Rachaneni S

Rachwan Rayan Jo

Racine Annie M

Radestad Ingela

Radke Peter

Radwan Mahmoud

Raffetto Joseph D

Rafieian-Kopaei M

Rafnsson Vilhjálmur 
Rafter Natasha

Ragonnet Romain

Ragupathi Loheetha

Rahman M Shafiqur

Rahman Abdul Rashid

Rahman Aminur

Rahman M

Rahman Mizanur

Rahman Nik Hisamuddin

Rahul Raj

Rai Ansaar

Rai Tapan

Raina Parminder

Raina Sunil Kumar

Raine Karen

Rainer Jennifer

Raisamo $\mathrm{Su}$

Raj Manu

Raja Edwin Amalraj

Rajaleid Kristiina

Rajan Sadna

Raji Hanieh

Rakhorst Hinne

Raknes Guttorm

Ram Felix

Ramacciati Nicola

Ramaekers Jan

Raman U

Ramautarsing Reshmie

Ramchand Rajeev

Ramonda Roberta

Ramos-Pichardo Juan

Ramos Delfina

Ramos Jorge

Ramos Jose M

Ramos Pedro

Ramoska Edward

Ranawaka Udaya

Rane Anil

Rangarathnam Balaji

Ranieri Veronica

Rankin Audrey

Rankin Judith

Rankin Nicole

Rankin Paul

Ranstam Jonas

Ranta Annemarei

Ranzani Otavio

Rao Amrita

Rao Prashanth

Rao Sunil

Rascati Karen

Raschke Robert A

Rasella Davide

Rasesemola Rm

Rashid Audil

Rasmussen-Barr Eva
Rasmussen Charlotte

Diana Nørregaard

Rasmussen Mette

Rasmussen Morten

Rasmussen Svein

Rasmussen Trine

Rasulo Domenica

Ratanawongsa Neda

Ratcliffe Julie

Rath Barbara

Rathod Sujit

Ratschen Elena

Raudino F

Rausch John

Ravara Sofia

Ravara Sofia B

Raven Melissa

Ravetti Cecilia Gomez

Ravi Parli R

Ravi Praful

Rawaf Salman

Rawshani Araz

Rawson Helen

Ray Samiran

Raychaudhuri SP

Rayman Margaret

Raymond Frederic

Raymond J

Rayner Christopher

Raynor David

Razai Mohammad

Razanamihaja Noeline

Reach Gérard

Rebar Amanda

Rebnord Ingrid

Rech Megan

Reddy Amanda L

Reddy Krishna

Redley Bernice

Redling Katharina

Redondo M

Redsell Sarah

Redshaw Maggie

Redwood Simon R

Reebye Uday

Reepalu Anton

Rees Charlotte

Rees Clare

Rees Nigel

Rees Vaughan

Reeve Emily

Reeve Emily

Reeves Mat

Reggiani Giulio

Marchesini

Regidor E

Regidor Enrique

Rehal Sunita
Rehn Marius

Reid Colin

Reid M

Reid Susan M

Reilly Marie

Reiner Zeljko

Reis Maria Fatima

Rejnö Gustaf

Relton Clare

Remer T

Remes Olivia

Remuzzi Andrea

Remuzzi Giuseppe

Remuzzi Giuseppe

Remy Linda L

Ren Kate

Renaudin Pierre

Rendle Kate

Rendo-Urteaga T

Renstrom Frida

Renwick Andrew

Renwick Laoise

Reppe Linda

Reppermund Simone

Resch Bernhard

Resche-Rigon Matthieu

Reupert Andrea

Reynaud Alexandre

Reynolds Charles

Reynolds Joanna

Reynolds Joshua C

Reynolds Penny

Rezwan Faisal

Rhee Chin Kook

Rhee Eun-Jung

Rhodes Elizabeth

Rhodes Jonathan

Rhon Daniel

Riaz Sadia

Ribeiro Daniel

Ribeiro Manny

Ribeiro Oscar

Ribeiro Rodrigo Antonini

Ribeiro Rosilene

Ribeiro Sonia

Ribera Josep-Maria

Ribo Marc

Ricca Valdo

Ricci Elena

Ricci Raffaella

Rice Henry

Rice LA

Rice Mary

Rice Tw

Rich Antonia

Richard Stephanie

Richards Toby

Richardson Annette
Richardson G Mark

Richardson Gerry

Richardson Lk

Richardson Noel

Riche Daniel M

Richmond Sarah

Richter Dirk

Richter Kneginja

Richter Nils

Rickard Claire

Rickwood Debra

Riddell Ca

Riddell Rebecca

Riddle Daniel

Ridley David

Ridout Deborah

Ridsdale Leone

Ridwan Sami

Riesgo Garcia Alba

Rigacci Luigi

Rigney Gabrielle

Rigotti Thomas

Rigters Sc

Riisgaard Helle

Rikard Robert

Riley Julia

Riley Malcolm

Riley Ruth

Riley Simon

Rim Kyung-Taek

Rimmele Thomas

Rimmer Joanne

Rimpelä Arja

Rinaldi Carmela

Ring David

Ring Liam

Ringholm Lene

Rios-Diaz Arturo

Ríos Antonio

Rios Domingos

Risch Lorenz

Rise Marit

Rising Kristin L

Riste Lisa

Ritchie Carrie

Ritchwood Tiarney

Ritsema $\mathrm{T}$

Ritt Martin

Rivas Carol

Rivera-Caravaca José Miguel

Rivers Emmanuel

Rizvi Syed

Rizzi Liara

Robben Paul

Robert Glenn

Roberts Christopher

Roberts David 
Roberts K

Roberts Megan

Roberts Robert

Roberts Seren Haf

Roberts Te

Robertson-Preidler J

Robertson Chris

Robinson David

Robinson James

Robinson Jude

Robinson Louise

Robinson Philip

Robinson Rebecca

Robinson Robert

Robinson Tom

Robinson Yohan

Robles-Garcia R

Robling Michael

Robson John

Robson Michael

Robson Paula

Roca Oriol

Rocha Luis

Rocha Marcia

Rocha Natalia

Rocha Viviane

Roche Tina

Rochon Paula

Ročka Saulius

Roda C

Rodda Simone

Rodgers Blake

Rodgers Lauren

Rodgers Peter

Rodilla Enrique

Rodin Danielle

Rodrigues Cm

Rodrigues I

Rodrigues Ian

Rodrigues Manuel

Rodrigues Rashmi

Rodrigues Veena

Rodriguez Robert M

Rodríguez Vm

Rodríguez López Santiago

Rodríguez-Caravaca G

Rodriguez-Esteban $\mathrm{R}$

Rodriguez-Galindo C

Rodriguez-Gay Carolina

Rodriguez-Llanes Jose Manuel

Rodriguez-Llanes Jose Manuel

Rodriguez-Monguio Rosa

Rodriguez-Morales AJ

Rodriguez-Moran M

Rodriguez-Padial Luis

Rodriguez-Roisin Roberto
Rodríguez-Violante Mayela

Rodriguez Cristina

Rodriguez Mariano

Roe Brenda

Roeder Falk

Roelants Mathieu

Roeters Van Lennep Jeanine

Roever Leonardo

Roffe Christine

Roffi Alice

Rogan Slavko

Rogers D

Rogers J

Rogers Rs

Rogerson Stephen

Roggenbuck Dirk

Rognås Leif

Rogozinska Ewelina

Roh Cheong-Rae

Rohloff Peter

Rohrmann Sabine

Røislien Jo

Rojas-Hernandez Cristhiam Mauricio

Rojas Graciela

Rokstad Anne

Roland Damian

Roland Martin

Rolfe M

Roll Stephanie

Rolle Udo

Rollefstad Silvia

Romandini Mario

Romano Claudio

Romero-Aroca Pedro

Ronaldson Kathlyn

Roncarolo F

Ronco Guglielmo

Roncoroni Julia

Ronellenfitsch U

Rong Pei-Jing

Rong Pei-Jing

Room Robin

Roos M

Roos Ronel

Roozen Sylvia

Ropponen Annina

Roque Matheus

Ros Emilio

Rosales Raymond

Rosati P

Rosdahl Jullia

Rose-Ackerman Susan

Rose A J

Rose Andre

Rose Miranda

Rose Olaf
Rosenbaum Sarah

Rosenberg Molly

Rosenberger Albert

Rosenblum Norman

Rosenstein Melissa G

Rosenthal Joe

Rosenthal Jorge

Rosenthal Meagen

Rosenthal Victor

Rosenvinge Jan

Roshanov Pavel

Rosier Peter F W M

Rosmalen Judith

Ross Jamie

Ross Mindy

Ross Samantha

Ross Sue

Rosselet Patricia

Rossi Filippo

Rossignol Jean-Francois

Rossiter Christine

Rossiter Nigel

Rossman Beverly

Rosso G

Roth Dominik

Rothman Michael

Rotter Iwona

Roujeau Jean-Claude

Roumeliotis Nadia

Round Thomas

Roush George

Rousham Emily

Rousseau Anne

Rousseau Cécile

Rousseau Marie-Claude

Rousset Sylvie

Roussey-Kesler G

Roustit Matthieu

Rovers John

Rovniak Liza

Row Jenny

Rowe Sarah

Rowe Rachael

Roy Ambuj

Roy Nobhojit

Roy S

Roze Des Ordons Amanda

Rozenberg Serge

Rozier Richard Gary

Ruan Zhizhong

Ruano-Ravina Alberto

Rubach M

Rubayi Salah

Rubin Daniel

Rubin Greg

Rubino C

Rubio-Valera Maria

Rubio Francisco
Rubis Pawel

Ruch Willibald

Rudin Rs

Rudolph James

Rue Montserrat

Ruff Ryan

Ruhnke Gregory

Ruilope Luis

Ruini Chiari

Ruiz-Ederra Javier

Ruíz Milagros

Ruiz Sarah

Ruland Cornelia

Rumbold Alice

Rungby Jørgen

Runhaar Jos

Runnacles Jane

Ruohola Aino

Ruppertsberg Alexa

Rurangirwa Andrew

Rushbrook Simon

Rusnak Martin

Russell F M

Russell Grant

Russell Michael

Russell Yvan

Russo Francesca

Russo Gianni

Russo Giorgio

Russo Giuseppina

Rutherford J S

Ruths Florian

Rutten Frans

Rutten Guy

Ryan Cormac

Ryan Karen

Ryan R

Ryan $\mathrm{T}$

Ryan Trevor

Ryckman Kk

Ryder Stephen

Ryff Carol

Rymaszewska Joanna

Ryoo Seung-Bum

Ryu Tomoki

Ryu Wi-Sun

Sa Zhihong

Saad Farid

Saad Mohamed

Saad Mohamed Nagy

Saarela Olli

Sabadell Jordi

Sabah Shiraz

Sabate J

Sabate Manel

Sabin Caroline

Sabo Roy

Sacco Simona 
Saccone Gabriele

Sachdev Perminder

Sadeghi Ramin

Sadeghi Roya

Sadler Lynn

Sadowski Marcin

Saedder Eva

Saeed Khalid

Saeki Keigo

Saemann Marcus

Saenger Michael

Saez Marc

Safari S

Saffari Seyed

Sagar Malvika

Saha Sanjib

Sahathevan Ramesh

Sahm Laura

Saibeni S

Saijo Masayuki

Saikia Anku Moni

Sainani Kristin

Saisho Yoshifumi

Saito Nobue

Saito Shota

Saji Naoki

Sajnani Nisha

Sajobi Tolulope

Sakakibara-Konishi Jun

Sakamaki Kentaro

Sakeah Evelyn

Sakellari E

Sakisaka Kayako

Sako Edward

Sakoda Lori

Saks Mike

Sakthong P

Sakuma Atsushi

Sakurada Yoichi

Salam-White Lialoma

Salamh Paul

Salas-Salvadó Prof Jordi

Salas-Vega Sebastian

Salavastru C

Salcher Maximilian

Salciccioli Justin

Saleem Fahad

Salgado Eva

Salinas Alberto

Salinero-Fort Miguel

Salisbury Chris

Sallustio Fabrizio

Salman Rustam

Salminen Paulina

Salobrar-Garcia Elena

Salonia Andrea

Saloojee Haroon

Saltman Richard
Saltychev M

Saltzman Darin J

Salvatore Stefano

Salvesen Kjell

Salzberger B

Sambhara S

Samos Matej

Sampaio Marcelo Santos

Sampalis John

Samsi Kritika

Samuel R

Sanak Marek

Sanchez Fernandez Ivan

Sanchez-Garcia Sergio

Sanchez-Guerrero Jorge

Sanchez-Salcedo Pablo

Sanchez-Solis Manuel

Sanchez Alvaro

Sanchez Carlos

Sandall Jane

Sandars John

Sandberg M

Sandberg Magnus

Sandblom Gabriel

Sandelowsky Hanna

Sander Lasse

Sandercock Gavin

Sanders Prashanthan

Sanders Taren

Sanderson M

Sandin Sven

Sandoval-Guzman Tatiana

Sandoval Claudio

Sandvik Hogne

Sanger Patrick

Sanjuan-Cervero R

Sanna Stefano

Sanna T

Sanneh Bakary

Santa Mina Daniel

Santacana Martí

Santaguida Pasqualina

Santamariña-Rubio E

Santana Rui

Santella Anthony

Santer Miriam

Santiago-Rivas Marimer

Santini Daniele

Santo Karla

Santos-Gallego Carlos

Santos-Lozano Alejandro

Santos Cristina

Santos Jose

Santos Lp

Santos Sara

Santosa A

Sanudo Borja

Sanz-Reig Javier
Sanz Jose

Sarafidis Pantelis

Saragiotto Bruno

Saramunee K

Sarant J

Saravelos S

Saraya Takeshi

Sarfo Fred

Sargent G M

Sargin Mehmet

Sarici Inanc

Sarin Enisha

Sarkar Chinmoy

Sarker Abdur R

Sarker Md Shafiqul

Sarki Ahmed

Sarkies Mn

Sarkola Taisto

Sarmiento Juan

Sarnowski Bettina

Sarosiek Shayna

Sarr Mg

Sarrami Pooria

Sarria-Santamera Antonio

Sarris Jerome

Sartelli Massimo

Sartorius Benn

Sasahara Shinichiro

Sasseville Maxime

Satherley Rose-Marie

Sathyanarayana Sheela

Sato A

Sato Izumi

Sato Naoki

Saugel Bernd

Saunders Charles

Saunders Carla

Saunders Patrick

Sauriasari R

Saut Ana

Savage C

Savastano Silvia

Saville Marion

Savoie-Roskos M

Savvas Steven M

Sawada Shin-Ichi

Sawas Tarek

Sax Hugo

Sayer John

Saywell Nicola

Scala-Bertola Julien

Scaldaferri F

Scallan Samantha

Scally Andrew

Scaringi Stefano

Schaan Bd

Schablon Anja

Schäfer Ingmar
Schaffer Andrea

Schälte Gereon

Scharfbillig Rw

Schechter A

Scheepers Renee

Scheers Hans

Scheinost Dustin

Scheller Bruno

Schenning $\mathrm{Kj}$

Scher Jose

Scherag Andre

Scherb Hagen

Scherbakov Nadja

Scherer Laura

Scherer Roberta

Scherer Roberta W

Scheuermann Taneisha

Schibler Kurt

Schiele Francois

Schiff Rebekah

Schiffl Helmut

Schiffman Mark

Schilling U M

Schiltz Nicholas K

Schlaich Markus

Schleyer Anneliese M

Schloesser Tom

Schlünssen Vivi

Schlünssen Vivi

Schlussel Michael

Schluter Philip

Schmidt Burkhard

Schmidt Río Valle

Jacqueline

Schmidt Amand Floriaan

Schmidt Axel J

Schmidt B

Schmidt Glen

Schmidt Heike

Schmidt Stefan

Schmidt Wolf-Peter

Schmiegelow Michelle

Schneditz Daniel

Schneider Antonius

Schneider Erika

Schneider Julie

Schneider Marguerite

Schneider Stefan

Schneider Sven

Schneider Wolfgang

Schneiderman Ju

Schoeller Soraia

Schoenborn Nancy

Schoenenberger Andreas

Schoepf U

Schofield P

Scholes Shaun

Scholl Isabelle 
Schöllgen Ina

Scholten Harm

Scholten Nadine

Schomerus Georg

Schöttker Ben

Schouten Jeroen

Schram Miranda

Schreiber Sanja

Schröder Sara Lena

Schroeder Je

Schuch Felipe

Schuessler Sandra

Schuh-Renner Anna

Schuit E

Schulte Phillip

Schultz Hans Henrik

Schultz Timothy

Schulz Amy

Schulz M

Schulz Martin

Schulz Peter

Schumacher Christine

Schüpbach Jörg

Schuster Kevin M

Schutte Stefanie

Schwab-Reese Laura

Schwartz Ann V

Schwartz Todd

Schwarzkopf Daniel

Schweiger Ulrich

Schwingshackl Lukas

Sciascia Savino

Scifres Cm

Scott Alex

Scott Fiona

Scott James

Scott Jason

Scott Jennifer

Scott Neil

Scott Paul A

Scoullar Michelle

Scribner Richard

Scrivener Katharine

Scullin Claire

Scullin Michael

Scuteri Angelo

Seabrook Jamie

Seacat Jason

Seale Tania

Seamark David

Seamon Mj

Searby Adam

Seaton Sarah

Sebastiani Giada

Seedat Yackoob

Seel Ron

Seeley Janet

Seeman Mary V
Segal Gad

Segal Leonie

Segurado Ricardo

Sehested Thomas

Seibold Petra

Seidu S

Seidu Samuel

Seino Yutaka

Seitz Dallas P

Sekelj Sandra

Sekhotha Mm

Sekula Peggy

Selamu M

Selby Kevin

Selby Nicholas

Seliga-Siwecka Joanna

Seligman Hilary

Seligman Laura

Selistre Luciano

Selleck Cynthia

Sellitti Df

Selmer C

Selove Rebecca

Selvarajah Dinesh

Semler Matthew

Sen Gupta Tarun

Sen Sanchita

Sen Srijan

Senan Suresh

Senanayake H

Sendlhofer Gerald

Seners Pierre

Sengul Sule

Sengupta U

Senn Stephen

Senore Carlo

Sentilhes Loïc

Seoane-Vazquez Enrique

Sepodes Bmn

Sepucha Karen

Sera Francesco

Sereda Magdalena

Serlachius Anna

Sermeus Walter

Serra-Majem Lluis

Serra Laura

Serra Monica

Serranheira Florentino

Serrao Sebastiano

Serri Karim

Sersa G

Servidio Rocco

Sethi Sanjay

Setiawan Agus

Setiawan D

Settnes Annette

Severini G

Sevil Javier
Sevincok Levent

Sewell Da

Sewell Daniel

Sewitch Maida

Sganga Federica

Shabanzadeh Daniel

Shabila Nazar

Shah Adil

Shah Alok

Shah Gh

Shah N

Shah Nilesh

Shah Nisar

Shah Prakesh

Shaha Kk

Shahhosseini Zohreh

Shahram Sana Z

Shahtahmassebi Golnaz

Shakespeare Tom

Shallcross Laura

Shamszad Pirouz

Shander Aryeh

Shang Hongcai

Shang Xianwen

Shankar Abhishek

Shankar Aparna

Shankardass Mala Kapur

Shannon Rosemary

Shanthanna Harsha

Shantsila Alena

Shao $\mathrm{H}$

Shapiro Allison

Shapiro Charles

Sharif-Askari F

Sharifi Vandad

Sharma Abhishek

Sharma Aditya

Sharma Akshay

Sharma Kathryn

Sharma Monisha

Sharma Nilima

Sharma Praveen

Sharma Ratika

Sharma Sudesh Raj

Sharma Sunjay

Sharma Tarang

Sharma Vijay K

Sharpe Darren

Sharples Edward

Sharpley Christopher

Shaukat Aasma

Shaw Elizabeth

Shaw Jonathan

Shaw Nicholas

Shaw Richard

Shaw William

Shea Cm

Sheard Laura
Shearer James

Sheaves Bryony

Sheehan Katie

Sheehan Connor

Sheehan M

Sheehan Orla

Sheehan Rory

Sheehan Rosemary

Sheen Aali

Sheets Debra

Shehabi Y

Sheikhattari P

Sheldon Trevor

Shen Minxue

Sheng Zhifeng

Sheperd James

Shepherd Andrew

Sheppard James

Sheppard Kelly W

Sheremata William

Shergis Johannah

Sherr Jennifer L

Sherrington Catherine

Sherwood James

Shewade Hemant

Shi Lin

Shi Zumin

Shibagaki Yugo

Shibahara Shigeki

Shibata Yoko

Shidhaye Rahul

Shields Beverley

Shields Le

Shields Michael

Shiell Alan

Shiffman Saul

Shiga Toshiya

Shigematsu Kazuo

Shikako-Thomas Keiko

Shikata Satoru

Shim Jae-Mahn

Shimokawa Hiroaki

Shimoyama Shouji

Shin Jinho

Shinozaki Tomohiro

Shinzato Márcia

Shiraishi Atsushi

Shirazian Shayan

Shiri Tinevimbo

Shirkey Beverly

Shirvani Marjan Ahmad

Shivaprasad

Channabasappa

Shoben Abigail

Shokri Azad

Shomron Noam

Shoptaw Steven

Short Camille 
Short Timothy

Shpitser Ilya

Shrestha Laxman

Shrestha Nabin

Shrestha Sumitra

Shub Alexis

Shukla Shakti D

Shultz James Michael

Shwe Mu Mu

Si Lei

Sia Drissa

Sibanda Euphemia L

Sibeoni Jordan

Sidani S

Siddiqi Maqsood

Siddiqui Taranum

Siddiqui Zakaria

Sidhu Mandeep

Sidorkiewicz Stéphanie

Siebenhofer Andrea

Siedner Mark

Siefken K

Siegel Martin

Siemieniuk Reed

Siering Ulrich

Sierra Monica

Siesky Brent

Siettos Constantinos

Sigmund Erik

Signal Louise

Signorelli S

Signori Alessio

Sihvonen Raine

Sikri Gaurav

Sil Asim

Silberstein Stephen

Silfverdal Sven Arne

Siljander Heli

Silke B

Silke Bernard

Silva Anabela G

Silvain Christine

Silventoinen Karri

Silver Michelle

Silver Sam

Silverwood Richard

Sim Jenny

Sim Julius

Simmer Karen

Simmering Jacob

Simmonds Mark

Simmons Ryan

Simms Victoria

Simón Carlos

Simon Gregory

Simon Steffen T

Simonds Laura

Simonetti Antonella
Simons Michael

Simpson G

Simpson Graham

Simpson Jodie

Simpson Judy

Simpson N

Simpson Steve

Sims Julian

Sindi S

Singh A

Singh Ankur

Singh Bb

Singh Chandra

Singh Harvinder

Singh Jasjit

Singh Jeffrey

Singh Kern

Singh Mongjam

Singh Pritam

Singh Ritesh

Singh Sangeeta

Singh Sonal

Sinha Anju

Sinha Dona

Sinha Madhumita

Sinha Rajeev

Siniscalchi A

Sinning C

Sinnott Carol

Sipe Theresa

Sips Ilona

Sipsma Heather

Siriphorn Akkradate

Siriwardena Ajith

Siriwardhana C

Sirois Caroline

Sit Regina Wing Shan

Sitch Alice

Sittig Dean

Sivamani Raja

Sivaprasad Sobha

Sivayoham Narani

Sizmur Steve

Sjogren Karin

Sjölander Maria

Skaane Per

Skånèr Ylva

Skelton Ja

Skelton John

Skene Simon

Skilton Michael

Skinner Margot

Skinner Timothy

Skivington Kathryn

Skjaerven Rolv

Skoch Jesse

Skogberg N

Skogstad Marit
Skovgaard Lene

Skrifvars M B

Skrobanski Hanna

Skrobik Y

Skrobik Yoanna

Skyttberg Niclas

Slade Anita

Sladek Ruth

Slater James

Slater Paul

Slaughter James

Slavin $\mathrm{Sj}$

Slawson Deborah

Sleijpen Marieke

Sliepen Maik

Slim Karem

Sloan Frank

Slobogean Gp

Slone Jeremy

Slootweg Irene

Slusher Tina

Slusky Danna A

Smalheiser Neil R

Smart Neil

Smedema Jan-Peter

Smeeth Liam

Smeraglia Francesco

Smidth Margrethe

Smith Lynette

Smith-Greenaway E

Smith-Osborne Alex

Smith-Turchyn Jenna

Smith Allan

Smith Andrea

Smith Andrew F

Smith Benjamin

Smith Clare

Smith Danielle

Smith David

Smith David

Smith Debbie

Smith Er

Smith Eric E

Smith Geoffrey P

Smith Ha

Smith James

Smith Jason

Smith Kelly

Smith Kimberley

Smith Laurie W

Smith Lesley

Smith Lesley

Smith Lindsay

Smith Margaret

Smith Mark

Smith Rebecca

Smith Ross C

Smith Steven M
Smith Susan

Smith Tenbroeck

Smith Toby O

Smith V

Smits Frans

Smylie Janet

Smythe Tracey

Snape Matthew

Sneyd Robert

Sniderman Ad

Snieder Harold

Snih Soham

Snoek Frank

Snoeker Ba

Snooks Helen

Snow Kathryn

Snowden Jonathan M

Snyder Christopher W

So Alexander

Soares-Oliveira M

Sobel Michael

Soberg Helene

Sobhan Sobhani

Soe Aung

Soeding P F

Soennichsen Andreas

Søgaard Mette

Sohn Min-Woong

Søholm Helle

Soilemezi Dia

Sola Augusto

Solberg Leif

Solebo Al

Soleimani Hussain

Solheim E

Soliman Nancy

Solini A

Sollid Stephen

Solomon Joel

Soltanpour Mohammad

Solvik Una

Solvoll Terje

Soma Yuki

Sommerfelt Halvor

Sommerlad Andrew

Sone $\mathrm{H}$

Sonet Ellen

Song Fujian

Song Jaejoon

Song Mingyang

Song Peige

Song Ruiguang

Song Soon

Soni Apurv

Sonnadara Ranil

Sonne David

Soo Hoo Soon

Sood Manish 
Sood Neeraj

Sood Seema

Soofi Sajid

Soong Christine

Soong Christine

Sorbye Ingvil

Sørbye Sveinung

Wergeland

Søreide Kjetil

Sorenson Claire

Sorg H

Sorlozano-Puerto Antonio

Sorrentino F

Soto Maria Teresa Solis

Sotoudeh Gity

Souliotis Kyriakos

Soundy Andrew

Souris Marc

Sousa David

Southwell Brian

Sovio Ulla

Sozmen Kaan

Spaak Jonas

Spaander Manon

Spada Francesca

Spadaccio Cristiano

Spain Da

Spain Debbie

Spain Victor

Spangaro Jo

Spaulding Aaron

Speed Ewen

Spelten Evelien

Spencer Frederick

Spencer Nick

Sperrin Matthew

Speth Robert

Spicer John

Spiegel David

Spielmans Glen

Spies Claudia

Spiewak Mateusz

Spijkeman M

Spikes Pilgrim

Spillane Ailbhe

Spinewine Anne

Spittle Alicia

Spooner Sharon

Sprague Courtenay

Sprague Sheila

Squires Jane

Sram Radim

Sreeramareddy

Chandrashekhar

Srivastava Neena

Srivastava Deo Kumar

Srivastava Kamna

Srivastava Padma
St Sauver Jennifer

St-Onge Marie-Pierre

Staals Julie

Stacey Gemma

Stacey Tomasina

Stafler Patrick

Staggs V

Stahl Daniel

Stahl Jp

Staiano Amanda

Stallknecht Bente

Stamatopoulos Nicole

Standfield L

Stange Jp

Stanhope V

Stanifer John

Stankiewicz JM

Stansfeld Stephen

Stansfield Brian

Stanton Josephine

Stanton Robert

Stanton Tasha

Stapelfeldt Christina

Staples Margaret

Staplin Natalie

Starkey Nj

Starkopf Joel

Stassen Patricia

Staub Kaspar

Stautz Kaidy

Stavropoulou C

Steadman Kathryn

Stebbins Julie

Stebbins Rebecca

Steben Marc

Steck Nicole

Steckelberg A

Steed Liz

Steel Emily

Steel Zachery

Steen Carlsson Katarina

Steens Anneke

Stefanescu Horia

Stefanutti Claudia

Stefler Denes

Stegbauer Constance

Stegers-Jager K

Stein Airton

Steinberg Benjamin A

Steinberg Gd

Steinberg Ho

Steinberg Nili

Steinemann Daniel

Steiner Jeffrey

Steiner Robert

Steingrimsson Jon A

Steinman Mike

Steinmetz Helmuth
Steinsbekk Aslak

Steiropoulos Paschalis

Stel Vianda

Steluti Josiane

Stempski Sarah

Stenehjem Jo

Stenfert-Kroese Biza

Stenman Emelie

Stensballe Lone Graff

Stephansson Olof

Stephen Elizabeth

Stephen J

Stephens Alexandre

Stephens Alisa Jane

Stephens Janna

Stephenson Anne

Stephenson Anne

Stephenson J

Ster Irina

Stergiou George

Sterling Sa

Steven Kathryn

Stevens Ac

Stevens Dennis

Stevens Gregory

Stevens Jennifer P

Stevens Kara

Stevens Natasha

Stevens Richard

Stevens Sarah

Stevenson Mark

Stevenson Ted

Stewardson Andrew J

Stewart-Brown Sarah

Stewart Arthur

Stewart Kay

Stewart Orion

Stewart Paul Michael

Stewart Robert

Stewart Robert C

Stewart Simon

Stickland Michael K

Stienen Mn

Stiggelbout Anne

Stirling Christine

Stitzel Joel

Stochkendahl Mette

Stockings Emily

Stockley Rachel

Stocks Nigel

Stockwell-Smith G

Stockwell David C

Stockwell Timothy

Stoddard Gregory

Stodel Emma

Stojkovic Tanya

Stokes Maria

Stokes Mark
Stokes Tim

Stoll Matthew

Stolper Erik

Stolwijk Carmen

Stolzenburg S

Stone Louise

Stone $\mathrm{Pw}$

Stoner L

Storm Marianne

Stortecky Stefan

Story David

Stover Angela

Støvring H

Strand Ta

Strand Tor

Strandberg Timo

Stranges Saverio

Straßner Cornelia

Straßner Cornelia

Stratton Irene

Stratton Irene

Stratton Samuel

Straus Sharon

Strauß Markus

Street Deborah

Street Maryann

Streeter Adam

Streit Sven

Strnad Luke

Strogatz David

Strom Joel

Strom T

Strong Larkin

Stronks H

Stronks Karien

Strum Stephen

Stuart Heather

Stuart Jennifer J

Stubbe Maria

Stuhec Matej

Sturgiss Elizabeth

Sturman Nancy

Sturmberg Joachim

Sturrock Andrew

Sturrock Bonnie

Stuttard Lucy

Stylianou Michalis

Stylianou Neophytos

Stynes Siobhan

$\mathrm{Su}$ Gl

Su Ting-Li

Subasi Abdulhamit

Subbe Chris

Subramaniam A

Subramaniam M

Subramanian Ahalya

Subramanian Janakiraman

Suchorska Bogdarna 
Sudfield Cristopher

Sudo Akihiro

Suen Yi-Nam

Suffoletto Brian

Sugano Kentaro

Sugaya Akiko

Sugumaran S

Sujan Mark

Suliga Edyta

Sulku Seher Nur

Sullivan Adam

Sullivan Clair

Sullivan Thomas

Sultana Farhana

Sumalinog Rafael

Suman Arnela

Summers Dominic

Sun Carolyn

Sun D

Sun Guanghao

Sun Jared

Sun Jian

Sun Jing

Sun Qi

Sun Qiang

Sun Yan

Sun Yingpu

Sun Zhong-Hua

Sundaram $\mathrm{Kr}$

Sundbøll Jens

Sundbom Fredrik

Sundbom Magnus

Sunderland Bruce

Sundling Vibeke

Sung Shih-Hsien

Suominen Sakari

Supamanee Treeyaphan

Suraya Abdul-Razak

Surdacki Andrzej

Sureshkumar K

Sutcliffe Kathleen

Sutcliffe Robert

Sutherland Rachel

Sutter-Dallay A

Sutton Andrew

Sutton Chris

Suurmond J

Suzuki Hidekazu

Suzuki Kohta

Suzuki Sho

Suzuki Shunji

Svartengren Magnus

Svensson Elisabeth

Svensson Jannet

Swaminathan Meenakshi

Swart Michael

Sweeney Kim

Sweeting Helen
Sweetland Annika

Swinkels Ilse

Swinkels Jan

Switaj Paul

Syamala Ts

Sydes Matt

Symon Brian

Symonds Raymond

Symons K

Synnot Anneliese

Szakmany Tamas

Székely Andrea

Szyszkowicz Mieczysław

Taal Maarten

Taavi Erkkola

Taber Jennifer

Tada Yuji

Taddio Andrea

Tadesse Birkneh

Tadic Marijana

Tadros Allison

Taghian Alphonse G

Taguchi Ayumu

Taheri Shahrad

Taimela Simo

Taing Meng-Wong

Tajima Emiko A

Takagi Michael

Takahashi H

Takahashi Hideto

Takahashi Masaya

Takahashi Shinichiro

Takamura Akiteru

Takamura Noboru

Takayama H

Takeda Takashi

Takemura Toshio

Takeuchi Hiroyoshi

Takura Tomoyuki

Takwoingi Yemisi

Talan David

Talas Melek Serpil

Talbert Alison

Talbot H Keipp

Taler Sandra

Talias Michael

Talpey Scott

Tam Wilson

Tam Yat Hung

Tambo Ernest

Tamez Hector

Tamiru Abreham

Tammemagi Martin

Tampah-Naah Anthony Mwinilanaa

Tan Amy C W

Tan Boon Yeow

Tan Garry
Tan Gavin

Tan Le Van

Tan Maw Pin

Tanaka A

Tanaka Koji

Tanaka Nobue

Tanaka Yoshiya

Tang Hsin-Yi

Tang Jason

Tang Jw

Tang Karen

Tang Lynn

Tang Min-Zhong

Tang N -J

Tang Shangfeng

Tang Sydney

Tang Victoria

Tang Xiangdong

Tanizaki S

Tao Chuanmin

Tao Hongbing

Tao Rong

Tapia Granados Jose A

Tappin David

Tarantino Giovanni

Tarasoutchi F

Tarassenko Lionel

Tarcan Menderes

Tariq Shema

Tarquinio D

Tastet Lionel

Tatari Hassan

Tate Anne

Tateishi Ryosuke

Tauger Nathan

Taulbut Martin

Taw Mb

Taye Dr Belynew Wassie

Taylor Allan

Taylor Caroline

Taylor Clare

Taylor David

Taylor Elizabeth

Taylor Gemma

Taylor Kelly

Taylor Matthew

Taylor Melanie

Taylor Michelle

Taylor Nicholas

Taylor Richard

Taylor Rod

Tayob Nabihah

Te Kronnie Geertruy

Teare Harriet

Teasdale Emma

Tebala Giovanni

Tecson Kristen

Teh Andrew W
Teh Jane

Teixeira Catarina

Teixeira $\mathrm{S}$

Tekwe Carmen

Telford Richard

Teljeur Conor

Telo Gabriela

Temming $\mathrm{P}$

Ten Hoor Gill A

Tenenbaum A

Teng Zhongzhao

Tengan Fatima

Tennyson Joseph

Tenovuo Olli

Ter Hoeve Nienke

Ter Horst Kasper

Terashima Mikiko

Terhune Kyla

Ternhag Anders

Terras Mm

Terry Robert F

Terwee Caroline

Tesfaye Markos

Tetsworth Kevin

Tettamanti Mauro

Teye-Kwadjo Enoch

Teyhan Alison

Thabet Abdel

Thabrew Hiran

Thacher Tom

Thackeray Anne

Thadani Udho

Thakur Megha

Thalib Lukman

Thandrayen Kebashni

Thangaratinam Shakila

Thankappan Kr

Thapa Subash

Thappa Devinder

Thavorn Kednapa

Theilke SM

Thelin Eric

Theodorou Vassilia

Theorell $\mathrm{T}$

Theorell Tores

Theou Olga

Thévenod Frank

Thiede Michael

Thigpen Jonathan

Thijs Vincent

Thilen $\mathrm{Sr}$

Thilo Christian

Thom Elizabeth

Thom H

Thomas Thomas

Thomas Chloe

Thomas Eileen

Thomas N 
Thomas Rae

Thomas Rl

Thomas Roger

Thomas Susan

Thombs Brett

Thompson Coon Jo

Thompson-Paul Angela

Thompson Ae

Thompson Claire

Thompson Ds

Thompson Jennifer

Thompson Jm

Thompson Lora

Thompson Matthew

Thompson Mjj

Thompson Neill

Thompson Richard

Thompson Stephanie

Thompson Wade

Thomsen Liv Cecilie

Thomson Richard

Thorley $\mathrm{K}$

Thorley Nicola

Thorlund Jonas

Thorn Joanna

Thorne Kymberley

Thornton Jane S

Thornton Jim

Thorslund Birgitta

Thorsteinsson Einar

Thrasher Jf

Thrift Amanda

Thuret Gilles

Tian Jingyan

Tibaldi Joseph

Tielsch James M

Tierney Sean

Tierney Stephanie

Tiffin Paul

Tighe Joseph

Tighe M

Tijssen M

Till Benedikt

Tiller Heidi

Timm Anja

Timmis Adam

Timmis Christopher

Timoteo Ana Teresa

Timpson Nic

Timulak Ladislav

Tin Tin Sandar

Tiongson Erwin R

Tippett Vc

Tiruneh Bewket

Tissing Wim

Titaley Christiana

Titman Andrew

Tizzano Dr Eduardo
Tobey Matthew

Tobias Deirdre

Tobiasch Moritz

Tobin Katy

Tobin Simon

Toch-Marquardt Marlen

Toddenroth Dennis

Toeteberg-Harms Marc

Toft Sørensen Henrik

Toft Mathias

Tohid Hizlinda

Tohkin Masahiro

Tokas Theodoros

Tolppanen Anna-Maija

Tomasdottir Margret

Tomasi Elaine

Tomczyk Samuel

Tomita Naoto

Tomiyama Hirofumi

Tomizawa K

Tomlinson George

Tomnay Jane

Tonetti Maurizio

Tong David

Tonin Fernanda

Tonkin-Crine $\mathrm{S}$

Tonkin-Crine Sarah

Tonna Antonella

Toomey Elaine

Toomey Sinead

Toozs-Hobson Philip

Top Karina

Torchin Heloise

Torgerson David

Torniainen Minna

Toronto Coleen

Torpey Kwasi

Torrance $\mathrm{H}$

Torregrosa Miquel

Torres-Borrego J

Torres Antoni

Torres Georgia

Torres Jacqueline

Torrey Prof Fuller

Torti Carlo

Touburg Giorgio

Touchette Daniel

Tovar A

Townson J

Toye Francine

Tra Joppe

Trabold Nicole

Tracy Lincoln

Tracy Melissa

Traeger Adrian

Traissac Pierre

Trak M

Tramonti Francesco
Tramper-Stranders

Gerdien

Tran Bx

Tran Duong

Tran Viet-Thi

Trani Jean-Francois

Trask Catherine

Traub Stephan

Trautmann Sebastian

Treadwell Jonathan

Treadwell Julian

Trehan Amita

Tremblay Dominique

Triadafilopoulos G

Trickey Amber W

Triga Maria

Trinh Quoc-Dien

Tripathi Vandana

Tripathi Vrijesh

Tripepi Giovanni

Tripp Dean A

Tritter Jonathan

Triunfo Stefania

Trottier Kathryn

Trousselard Marion

Trovik Jone

Troya Isabela

Trtiunfo S

Trudel-Fitzgerald Claudia

Trueger Seth

Truong Tony

Trygg Nadja

Trzciński K

Tsai Chia-Liang

Tsai Der-Chong

Tsai Jeffrey Che-Hung

Tsang St

Tsao Cm

Tsapas Apostolos

Tsapepas Demetra

Tschandl Philipp

Tseh Wayland

Tseng Hsu-Min

Tsenkova Vera

Tsikopoulos Konstantinos

Tsikrika Stamatoula

Tsilidis Konstantinos K

Tsivgoulis Georgios

Tso Simon

Tsochatzis E

Tsoi Kelvin Kf

Tsokos Chris $\mathrm{P}$

Tsourtos George

Tsuchiya Asuka

Tsuga Kazuhiro

Tsuji Taishi

Tsujimoto Tetsuro

Tsuruya Kazuhiko
Tu Dongsheng

Tu Jack

Tuccori Marco

Tucker Patricia

Tucker Sharon

Tuddenham Sa

Tudor Car Lorainne

Tul N

Tuleasca Constantin

Tuleta I

Tume Lyvonne

Tuncil Yunus

Tung El

Tung Ericka E

Tung Tao-Hsin

Tuomisto Jouko

Tupasi Thelma

Tupesis Janis

Tura Abera Kenay

Turgerman Gadi

Turgut Burak

Turnbull Christopher

Turner Grace

Turner Janette

Turner John

Turner Jonathan

Turner Michael

Turner Rebecca

Turner Sheila

Turner William

Turok Dk

Turrini Fabrizio

Tuttle Michael

Tuttolomondo A

Tuttolomondo Antonino

Tutton Elizabeth

Tuzcu Ayla

Twigg Liz

Twigg Michael

Tyler Richard

Tyson Sarah

Tzimas Petros

Tziomalos Konstantinos

Ubbink Dirk T

Uchihara Toshiki

Uddin Shahadat

Udler Miriam

Ugarte-Gil Mf

Uguz F

Uhlhaas $\mathrm{Pj}$

Uijen Annemarie

Ukah Uv

Ukoumunne Obioha

Uleberg Oddvar

Ulfvengren Pernilla

Ulovec Zlatko

Ulvik Rj

Umakanthan Jayadev 


\section{Umbro I}

Umemura Toshitaka

Underwood Martin

Unnanuntana Aasis

Unnikrishnan B

Upadhyay Ashish

Uphoff E

Uprety Dipesh

Upshur Carole

Urbano Javier

Urbano Richard

Urbanos Rosa

Urben Sebastien

Urquia Marcelo L

Urushibara Noriko

Urzúa Alfonso

Usami Eiseki

Usher-Smith Juliet

Utens Cecile

Uthman Olalekan

Uttley Lesley

Uusi-Rasi Kirsti

Uzuner $\mathrm{N}$

Vaes Anouk

Vaes Bert

Vafaei Afshin

Vain N

Väisänen Heini

Vaithianathan Rhema

Vajro Pietro

Valable Samuel

Valaitis Ruta

Valdmanis Vg

Vale Diama Bhadra

Valente R

Valery $\mathrm{P}$

Valgimigli Marco

Valgimigli Marco

Valier Helen

Vallejo-Ruiz V

Valliant Amanda

Vallurupalli Srikanth

Valtis Yannis

Van Amelsvoort Ludovic

Van Ballegooijen Wouter

Van Bergen Jan

Van Beuzekom M

Van Blarigan Erin

Van Boven Kees

Van Calster Ben

Van Criekinge Tamaya

Van Den Akker-Van Marle ME

Van Den Bemt Patricia

Van Den Berg-Vos Rm

Van Den Berg Merel

Van Den Berge Minke JC

Van Den Bogert Cornelis
Van Den Bossche Tobi

Van Den Brand Crispijn

Van Den Bruel Ann

Van Den Bussche Hendrik

Van Den Ende Cornelia

Van Der Cingel Margreet

Van Der Does Y

Van Der Eerden Mm

Van Der Gaag Rutger Jan

Van Der Glas Hilbert

Van Der Graaf Wt

Van Der Haar Frits

Van Der Hulst R

Van Der Kuyl Antoinette

Van Der Laan Danielle

Van Der Meer Jos

Van Der Schaaf Marieke

Van Der Steen Jenny

Van Der Voort Peter

Van Der Weegen Walter

Van Der Wees Philip

Van Der Werf Mj

Van Der Wouden

Johannes

Van Der Zanden Tjitske

Van Der Zwaard Babette

Van Der Zweerde Tanja

Van Dijk - De Vries Anneke

Van Dijk Monique

Van Dongen Anne

Van Dulmen S

Van Gelder Marleen

Van Grootven Bastiaan

Van Hamel C

Van Haren Frank

Van Harten Wim

Van Heijst Arno

Van Hillegersberg Richard

Van Hoye A

Van Huis M

Van Ijzendoorn Marjolein $\mathrm{MCO}$

Van Jonbergen Hp

Van Lanschot Jan

Van Leeuwen Flora

Van Leeuwen Marieke

Van Lissa $\mathrm{Cj}$

Van Marwijk H

Van Marwijk Harm

Van Mourik Maaike

Van Naarden Braun Kim

Van Oeveren W

Van Oostwaard Miriam

Van Osch Frits

Van Oyen H

Van Poppel M

Van Rooyen J M

Van Rosendael Ar
Van Roy Kaatje

Van Schaardenburg

Dirkjan

Van Schayck Onno

Van Sluijs Esther

Van Stavern Gregory P

Van Summeren Jojanneke

Van Thriel C

Van Tubergen Am

Van Veen Evert-Ben

Van Velzen Judith

Van Weel Chris

Van Wegen E

Van Wely Madelon

Van Wijk Gerth

Van Wormer Katherine

Van Wyk Andoret

Van Wyk Lizelle

Van Wyk Paula

Van C

Vanacore Nicola

Vance David

Vandelanotte Corneel

Vandeleur Caroline

Vanderkruik Rachel

Vanderloo Leigh

Vandermeulen Corinne

Vandersteegen Tom

Vanderwall Cassandra

Vandormael Alain

Vangilder C

Vankim Nicole

Vanky Eszter

Vanmassenhove Jill

Vannucchi M G

Vanramshorst G

Vanthienen Jan

Varadhan R

Vardasca R

Varenne Benoit

Varghese B

Varghese Beena

Varghese Ron

Varnfield Marlien

Vartiainen Erkki

Vasan Senthil

Vasiliki B

Vassalle Cristina

Vassilatou E

Vasudevan Erin

Vatsayan Anant

Vatten Lars

Vaucher Paul

Vaughan Sue

Vavilala Monica

Vaz L

Vazquez Benitez Gabriela

Vazquez Fernando
Vecoli Cecilia

Vedi Aditi

Vedio A

Veelo Denise

Veerman Lennert

Vega Tomás

Velasco Patricia

Vellakkal Sukumar

Velleman Y

Vellinga Akke

Vellios Nicole

Vemuri Prashanthi

Venderbos Lionne D F

Venekamp Roderick

Venetis Chris

Venkatesan Arun

Venkatesh Arjun

Venners Scott

Ventimiglia Eugenio

Vera-Hernandez Marcos

Verbakel Jan

Verbeek Marcel

Verberk Willem

Verbiest Marjolein

Vercauteren Suzanne M

Vercellini Paolo

Verd Sergio

Verhagen Arianne

Verheij Robert A

Verhoeven Bas

Verlaan S

Verma Madhur

Verma Manisha

Verma Puja

Vermeir Peter

Vermeire Katrien

Vernay Michel

Vernon Howard

Verroken Charlotte

Verschoor Chris

Vervloet Marcia

Verykouki Eleni

Vestita Michelangelo

Viasus Diego

Vickers Andrew

Victoor Aafke

Victoria Pendon-Ruiz De Mier M

Vida Robert G

Videhult Pierre Pernilla

Viegi Giovanni

Vieira A

Vieira Edgar

Vieira Rute

Viergever Roderik

Vigue Bernard

Vik Eline

Vilchinsky Noa 
Villa Gianluca

Villalonga-Olives E

Villanueva-Vilchis Maria

Villar Jesús

Villareal Maria

Villari Paolo

Vincent Charles

Vincent Jean-Louis

Viney Kerri

Viney Rowena

Vinikoor Michael

Vink Maarten

Vinnikov Denis

Vinogradova Yana

Vinturache Angela

Violan-Fors Concepción

Viotti Sara

Virgili Gianni

Virues-Ortega Javier

Visram Shelina

Visser Marieke

Viswanathan S

Vitagliano Amerigo

Vitoratou S

Vitoria Paulo

Vitry Agnes

Viveros-Sandoval Martha

Ramsden Vivian R

Vizcaychipi M

Voeller Heinz

Vogel C

Voigt-Radloff Sebastian

Volken Thomas

Vollmer Robin T

Vollrath Margarete Erika

Volovici Victor

Von Bonsdorff Mikaela

Von Dem Knesebeck Olaf

Von Kries Ruediger

Vonthein Reinhard

Vorderwulbecke Florian

Vos Alinda

Vos Rimke C

Voss M H

Vossen Matthias

Vossius Corinna

Vozoris Nicholas

Vrbnjak Dominika

Vrijens Bernard

Vrijkotte Tanja

Vucinic Violeta

Vugts Miel

Vujcic Isidora

Vuoskoski Pirjo

Vural Aslı

Vyas Sangita

Vykuntaraju K

Wachman Em
Wachob David

Wada Hideo

Wada Kyoko

Wada Shinichi

Wade Angie

Wade Dorothy

Wade Julia

Wadhwa Rachna

Waeber Bernard

Wærsted Morten

Wagenlehner F

Wager Elizabeth

Wagg Adrian

Wagner Abram L

Wagner Jamie

Wahab Stéphanie

Wahl Hans-Werner

Wahl Michael

Waigankar Ss

Wainwright Megan

Wakefield Juliet

Walbeek Corne

Wald Heidi

Wald Ron

Waldmann Carl

Waldum $\mathrm{Hl}$

Walker Blake Byron

Walker Ck

Walker Jennifer

Walker Jeremy

Walker Marion

Walker R

Walker R

Walker Rachael

Walker Richard

Walkom Emily

Wall Patrick

Wallace J

Wallace Emma

Wallace Euan

Wallace Jacqueline

Wallace Maeve

Wallace Paul

Wallen Margaret

Wallen Matthew

Waller Jo

Waller Michael

Wallin Lars

Wallin Mitchell T

Wallis Lee

Wallis Lee

Wallis Thomas S A

Walsh Ka

Walsh Timothy

Walsh William

Walter Matthias

Walter Scott

Walters Robin
Walters E Haydn

Walters Stephen

Walthall Helen

Walton-Moss Benita

Walton Merrilyn

Walton Rebecca

Walton Shelley F

Wand Handan

Wändell Per

Wanderley Carlos

Wandersman Abraham

Wang B

Wang Chen-Ping

Wang Chenchen

Wang Chongjian

Wang Deyun

Wang Dongqing

Wang Duolao

Wang Hai-Long

Wang Haolu

Wang Hongyue

Wang Jinfeng

Wang Jing-Houng

Wang Kai

Wang Liang

Wang Ling

Wang Lu

Wang Ningli

Wang Pei

Wang Peizhong

Wang Qing

Wang Tao

Wang Wei

Wang Wenru

Wang Xi

Wang Xia

Wang Xiaobin

Wang Y

Wang Y-X

Wang Yao-Gang

Wang Yilong

Wang Yufu

Wang Zhen

Wang Zhuo

Wanger Adam

Wangmo Tenzin

Wannamaker Braxton

Wanner Philippe

Ward Leigh C

Ward Darian

Ward Kt

Ward Lesley

Ward Mary

Warmoth Krystal

Warren Charlotte

Warren F

Warren Fiona

Warren N
Warren Rueben

Wartman Steven

Warwick David

Wasay Mohammad

Waseem Z

Wass Hanna

Wasser Tobias

Wasson J

Wasunna Aggrey

Waterman Heather

Waters Erica

Waterson Patrick

Waterworth Susan

Watkins Da

Watkins David

Watmough Simon

Watson Eila

Watson Julie

Watson Kathleen

Watson N

Watson Paula

Watson Peter

Watson Roger

Watson Samuel

Wattal Chand

Watts Caroline

Watts Sw

Watts T

Waugh Norman

Wawrzyniak Andrew

Weaver Marcia

Webb Girard Amy

Webb David

Webb Richard

Webber Jonathon

Webber Laura

Webel Allison R

Weber Jan

Weber Lutz

Weber Michael

Webster Joan

Webster Ruth

Wee Liang

Weeks Andrew

Weeks Gregory

Weeks Wb

Weems Carl

Weenink Jan-Willem

Weerasinghe Dona

Wegrzynek Paulina

Wegwarth Odette

Wehbe Tarek

Wehberg Sonja

Wehling Peter

Wei Shu-Qin

Wei Wei

Wei You-Dong

Weich Scott 
Weidemann Darcy

Weihs Martin

Weijers Rn

Weil Zachary

Weimer Melissa

Weinbroum Avi A

Weiner $\mathrm{C}$

Weinhouse Gerald

Weiniger Cf

Weinstein Maya

Weintraub Rebecca

Weis J

Weiser Thomas

Weiss Nicolas

Weiss Scott L

Welbourn Richard

Welch Cathy

Welch Vivian

Weller Jennifer

Wellesley Diana

Wellman Robert

Wells Ellen

Wells Katerina

Wellwood Ian

Welp Annalena

Wen Kuang-Yi

Wen Zehuai

Wendelboe Aaron

Wentzel-Larsen Tore

Weobong Benedict

Werdan Karl

Werner Erik

Wershofen Birgit

Wertheimer Albert

Wesonga $\mathrm{R}$

Wessel Jennifer

West Robert

West Sophie

Westby Marie

Westerink J

Westgate $\mathrm{P}$

Wettergren L

Wetterslev Jorn

Wheat Chelle

While Alison

Whitaker Katriina

White Helen

White Jacquie

White James

White Marc

White P

White Patience

White Paul

White Paul F

White Sarah

White Victoria

Whitehead Kirsten

Whitehead Lisa
Whitehead Margaret

Whitehouse Michael

Whitehouse Peter

Whitehouse Sarah

Whitford Kevin

Whitham Diane

Whiting Penny

Whitney Julie

Whitson Heather

Whittaker G

Whittaker William

Whop Lisa

Whybrow P

Whysall Zara

Whyte Em

Wi Chung-Il

Wiart L

Wiberg Michael

Wichaidit W

Wichman Christopher

Wichmann Dominic

Wicki Matthias

Wickrama K

Wicks Paul

Widdowson Marc-Alain

Widger John

Widyahening Indah

Widyandana Doni

Wiedermann Ursula

Wiedermann W

Wiegers Trees

Wieland L Susan

Wieldraaijer Thijs

Wiener-Ogilvie Sharon

Wienert J

Wiens Matthew

Wieringa Sietse

Wiernik Emmanuel

Wiese-Posselt M

Wiest Michelle

Wiewel Ew

Wijeratne Tissa

Wijeysundera Duminda

Wijnhoven Hanneke

Wikswo Mary

Wilby Kyle

Wilder-Smith A

Wildevuur Sabine E

Wiles Rebecca

Wilk Adam S

Wilkins Chris

Wilkins Kenneth

Wilkinson Chris

Wilkinson Claire

Wilkinson Lua

Wilkinson Tom

Willaing Ingrid

Willems Wj
Willey Barbara

Williams Amanda

Williams Annie

Williams Christine

Williams Christopher

Williams Dr Cylie

Williams J

Williams Joah

Williams John

Williams John

Williams Kim

Williamson David

Williamson Wilby

Willis Andrew

Willis Eileen

Willis Erin

Wilson Amy

Wilson Andrew

Wilson Andrew

Wilson Andrew

Wilson Andrew

Wilson Edward

Wilson Elena

Wilson Helen

Wilson Louise F

Wilson Mark

Wilson Nairn

Wilson Paul

Wilunda Calistus

Win Hla Hla

Win Aung

Winchester Chris

Windham Beverly G

Winer Rachel

Wingfield Tom

Winichagoon Pattanee

Winje Brita

Winkel Bo Gregers

Winkley Kirsty

Winslade Mathew

Winston $\mathrm{Bw}$

Winter Samantha

Winters Brian

Wirleitner Barbara

Wirth Janine

Wischmann Tewes

Wise Lauren

Wisorg Torben

Wister Andrew

Withers Mellissa

Witkop M

Witt Daniel

Witt Karsten

Wittmann Miriam

Witzke Kara

Wiysonge Charles

Wnent Jan

Woelk Godfrey
Wojdyla Daniel

Wolfe Charles

Wolfe Pj

Wolff Kristina

Wolff Robert

Wolfler Andrea

Wolk Courtney

Wolstenholme Daniel

Wolters Lidewij

Wong Cho Lee

Wong Cynthia

Wong Emily S

Wong Geoff

Wong Geoffrey

Wong Io

Wong Li Ping

Wong Muh-Geot

Wong Patricia

Wong Peter

Wong Sabrina

Wong Samuel

Wong Te

Wong Toh Yoon Ezekiel

Wong Victoria

Wong Vincent

Wong Yiu Ming

Wood Damian

Wood Fiona

Wood Rachael

Woodbury Anna

Woodhead Mark

Woodhouse Margaret

Woodman Richard

Woodside Jayne

Woodward Mark

Woolcott C G

Woolf Katherine

Woolfall Kerry

Worm Margitta

Worodria William

Worrall Linda

Woubishet Woldeamanuel

Yohannes

Woudstra Anke

Wouters Hans

Wouters Olivier

Woznitza Nicholas

Wright-St Clair Valerie

Wright Alison

Wright Charlotte

Wright David

Wright Eric

Wright Frances

Wright Penny

Wright Sally

Wu Ai-Min

Wu C

Wu Chen-Long 
Wu Chenkai

Wu Dan

Wu Dongfeng

Wu Fred M

Wu Huiyun

Wu Jing

Wu Kongming

Wu Qinghua

$\mathrm{Wu} \mathrm{Sg}$

Wu Teddy

Wu Xinyin

Wu Xinyin

Wu Yangfeng

Wu Zunyou

Würtz Else

Wyder M

Wye Lesley

Wynne-Jones Gwenllian

Wynter Karen

Xi Bo

Xi Yin

Xia Yinglin

Xian Ying

Xiang B

Xie Shaohua

Xing Jianmin

Xiong Linping

Xiong Xu

Xirasagar Sudha

$\mathrm{Xu}$ Aijun

Xu Fei

Xu Fenglian

Xu Gang

Xu Hongwei

Xu Hongyan

Xu Jiaqiong

Xu Lingzhong

Xu Shifen

Xu Xianglong

Xu Xiaolin

Xu Zhenghao

Xu Zhiwei

Xue Baowen

Xue Di

Xue Ying

Yabe Daisuke

Yaeger Rona

Yakoob Mohammad

Yakoot Mostafa

Yamada $\mathrm{S}$

Yamakawa Hideaki

Yamamoto $\mathrm{M}$

Yamanaka H

Yamato T

Yamaue Hiroki

Yamaya Mutsuo

Yan Ll

Yan Shiyan
Yan Weili

Yang Kuen-Cheh

Yang Fan

Yang Feng

Yang Hongmei

Yang Jie

Yang Jingyun

Yang Jinqiu

Yang Juan

Yang L

Yang Lin

Yang Qing

Yang Shaoping

Yang $\mathrm{T}$

Yang Tingzhong

Yang Won Jae

Yang Xinjian

Yang Yen

Yang Yin-Jian

Yano Yuichiro

Yao Hai-Mu

Yao $\mathrm{T}$

Yao Yingshui

Yap Christina

Yarlagadda Naveen

Yasar Sevil

Yasin Siti Munira

Yasuda S

Yates Deborah

Yates Sean

Yavchitz Amelie

Yavuz S

Yazbek Sohar

Ye Wen

Yeap Swan Sim

Yeates Keith

Yeboah J

Yeh Fawn

Yeh Justin

Yeh Yueh-Chiao

Yelland Jane

Yen Cheng-Fang

Yen Hung-Rong

Yeo $\mathrm{Hl}$

Yi Denise

Yih Katherine

Yim Hyun Woo

Yin Lei-Miao

Yin Zenong

Ylöstalo P

Yokomichi Hiroshi

Yokota Kei

Yokoyama H

Yokoyama Yoko

Yokoyama Yukari

Yong A

Yoo Ji Won

Yoon Jinsung
Yoon Yoonjin

Yorek Mark

Yorifuji Takashi

Yoshimura Reiji

You Hua

You Je Sung

You Myoungsoon

Youlden Danny

Younan Duraid

Young Amanda

Young Amber

Young Jeremy

Young Kenneth

Younger Alastair

Yousafzai Aisha

Yu Catherine

Yu Fang-Fang

Yu Hongjian

Yu Minbin

Yu Ming-Chih

Yu Shenyuan

Yu X

Yu Xue Qin

Yuan Shasha

Yuan Yan

Yuang Gonghuan

Yuchi Yixing

Yue Qingxi

Yuen $\mathrm{P}$

Yuguero Oriol

Yumoto Tetsuya

Yung Matthew

Yusif Salifu

Yusufali Afzalhussein

Zacharoff Kevin

Zacharowski Kai

Zacharski Leo

Zack Matthew

Zadnikar Monika

Zadow Amy

Zadpoor Aa

Zafón C

Zakaria Nassani

Mohammad

Zakariassen Erik

Zakarija-Grkovic Irena

Zakrzewska Joanna M

Zaman Huri Hasniza

Zaman Azfar

Zaman Mm

Zambon Antonella

Zamora Javier

Zandi Mohammad

Zanetti Hugo

Zangiacomi Martinez

Edson

Zaniboni Siqueira Batista

Keila
Zanini Claudia

Zaninotto Ana Luiza

Zanjani $\mathrm{F}$

Zannini Lucia

Zanolla Luisa

Zarogoulidis $\mathrm{P}$

Zayas Cilia

Zeber John E

Zebrack Brad

Zeeb Hajo

Zegers-Hochschild F

Zegers Marieke

Zeggwagh Amine Ali

Zehbe Ingeborg

Zeitlin Jennifer

Zeitoun Jean-David

Zeller Scott L

Zembe-Mkabile Wanga

Zembrzuski Verônica

Zeng Irene

Zeng Wu

Zeng Y

Zeng Zhen

Zenteno-Cuevas R

Zeps Nikolajs

Zerbo Ousseny

Zevin Boris

Zghebi Salwa

Zhai Guangju

Zhai Xiao

Zhan Tingting

Zhang Xin

Zhang Bing

Zhang D

Zhang Dongmei

Zhang Furen

Zhang James

Zhang Jianduan

Zhang Jing

Zhang Jun

Zhang Junhua

Zhang Lin

Zhang Liying

Zhang Melvyn

Zhang Mingmig

Zhang Nanhua

Zhang Qiwei

Zhang Xiaoju

Zhang Xin

Zhang Xing

Zhang Xingyu

Zhang Y

Zhang Ying

Zhang Yuan

Zhang Zhiyong

Zhang Zilu

Zhao A

Zhao Fang 
Zhao Fang-Fang

Zhao Jiwei

Zhao Li

Zhao Yinshan

Zhao Yuejen

Zhaokang Yuan

Zhaoxiang Bian

Zhelev Zhivko

Zheng Danni

Zheng Guo-Qing

Zheng Guoqing

Zheng Zhen

Zhiyong Peng

Zhong Hua

Zhong Lai-Ping

Zhou Chengchao
Zhou Jian-Da

Zhou Ping

Zhou Xinyu

Zhou Xudong

Zhou Zhongliang

Zhou Zhongliang

Zhu Dawei

Zhu E

Zhu Fengcai

Zhu Jiawen

Zhu Jiming

Zhu Ling

Zhu Minmin

Zhu Shijun

Zhu Wei

Zhu Zhiming
Zhuang Xun

Ziai Wendy

Zibold Felix

Ziegler Ekhard E

Ziemssen F

Zieschang Tania

Zigeuner R

Zigler Cory

Zikmund-Fisher Brian J

Zilberberg Marya

Zimerman Leandro

Zimmerli Werner

Zimmerman Emily B

Zimmerman Peta-Anne

Zimmermann Thomas

Zimmo Lena
Zis Panagiotis

Zisko Nina

Zissis A

Zitman Fg

Zolfaghari Parjam S

Zombré D

Zomer Ella

Zonderland Me

Zuckerman Diana

Zuo Yunxia

Zürcher Kathrin

Zurynski Yvonne

Zwaan Laura

Zwahlen Marcel

Zwank Michael

Zyoud Sa'ed 